ALEA, Lat. Am. J. Probab. Math. Stat. 17, 901-991 (2020)

DOI: $10.30757 /$ ALEA.v17-35

\title{
Analytic-geometric methods for finite Markov chains with applications to quasi-stationarity
}

\author{
Persi Diaconis, Kelsey Houston-Edwards and Laurent Saloff-Coste \\ Departments of Mathematics and Statistics, Stanford University \\ 390 Serra Mall, Stanford, CA 94305 USA \\ E-mail address: diaconis@math.stanford.edu \\ The Olin College of Engineering \\ 1000 Olin Way, Needham, MA 02492 USA \\ E-mail address: kedwards@olin.edu \\ Department of Mathematics, Cornell University \\ 567 Malott Hall, Cornell University, Ithaca, NY 14853, USA \\ E-mail address: lsc@math.cornell.edu
}

Abstract. For a relatively large class of well-behaved absorbing (or killed) finite Markov chains, we give detailed quantitative estimates regarding the behavior of the chain before it is absorbed (or killed). Typical examples are random walks on boxlike finite subsets of the square lattice $\mathbb{Z}^{d}$ absorbed (or killed) at the boundary. The analysis is based on Poincaré, Nash, and Harnack inequalities, moderate growth, and on the notions of John and inner-uniform domains.

\section{Contents}

1. Introduction $\quad 878$

1.1. Basic ideas and scope $\quad 878$

1.2. The Doob-transform technique 880

1.3. The 45 degree finite discrete cone 881

1.4. A short guide 883

2. John domains and Whitney coverings 885

2.1. John domains 887

2.2. Whitney coverings 892

3. Poincaré and Nash inequalities 895

3.1. Edge weights, associated Markov chains and Dirichlet forms $\quad 895$

$\begin{array}{ll}\text { 3.2. Poincaré inequalities } & 896\end{array}$

3.3. Nash inequality 898

4. Poincaré and $\mathcal{Q}$-Poincaré inequalities for John domains 899

4.1. Poincaré inequality for John domains 900

Received by the editors June 10th, 2019; accepted September 15th, 2020.

Key words and phrases. Markov Chains, Harnack Inequality, inner-uniform domains. 
4.2. Q-Poincaré inequality for John domains 906

5. Adding weights and comparison argument

5.1. Adding weight under the doubling assumption for the weighted measure

5.2. Adding weight without the doubling assumption for the weighted measure

5.3. Regular weights are always controlled 913

6. Application to Metropolis-type chains 914

6.1. Metropolis-type chains $\quad 914$

6.2. Results for Metropolis type chains 915

6.3. Explicit examples of Metropolis type chains 918

7. The Dirichlet-type chain in $U \quad 920$

7.1. The general theory of Doob's transform 921

7.2. Dirichlet-type chains in John domains 926

8. Inner-uniform domains 932

8.1. Definition and main convergence results 932

8.2. Proofs of Theorems 8.9 and 8.13: the cable space with loops 938

8.3. Point-wise kernel bounds 945

9. Some explicit examples 948

9.1. Graph distance balls in $\mathbb{Z}^{2} \quad 949$

9.2. $B(N) \backslash\{(0,0)\}$ in $\mathbb{Z}^{2} \quad 950$

9.3. $B(N) \backslash\{0\}$ in $B(N)$, in dimension $d>1 \quad 952$

9.4. $B(N) \backslash B_{2}(L)$ in $B(N)$, in dimension $d>1 \quad 953$

9.5. $B(N) \backslash B(L), d=2 \quad 956$

10. Summary and concluding remarks 958

$\begin{array}{ll}\text { Acknowledgements } & 959\end{array}$

Appendix A. Convex sets in $\mathbb{Z}^{d}$ with $d>2 \quad 959$

Appendix B. Volume doubling and moderate growth 961

B.1. Doubling and moderate growth $\quad 961$

$\begin{array}{ll}\text { References } & 964\end{array}$

\section{Introduction}

1.1. Basic ideas and scope. Markov chains that are either absorbed or killed at boundary points are important in many applications. We refer to Collet et al. (2013); Diaconis and Miclo (2015); Burkholder (1977); DeBlassie (1987); Denisov and Wachtel (2015) for entries to the vast literature regarding such chains and their applications. Absorption and killing are distinguished by what happens to the chain when it exits its domain $U$. In the killing case, it simply ceases to exist. In the absorbing case, the chain exits $U$ and gets absorbed at a specific boundary point which, from a classical viewpoint, is still part of the state space of the chain. In this paper we study the behavior of chains until they are either absorbed or killed, which means that there is no significant difference between the two cases. For simplicity, we will phrase the present work in the language of Markov chains killed at the boundary.

The goal of this article is to explain how to apply to finite Markov chains a well-established circle of ideas developed for and used in the study of the heat 
equation with Dirichlet boundary condition in Euclidean domains and manifolds with boundary, or, equivalently, for Brownian motion killed at the boundary. By applying these techniques to some finite Markov chains, we can provide good estimates for the behavior of these chains until they are killed. These estimates are also very useful for computing probabilities concerning the exit position of the process, that is, the position when the chain is killed. Such probabilities are related to harmonic measure and time-constrained variants. This is discussed by the authors in a follow-up article Diaconis et al. $(2020+)$. As a bonus, these techniques provide new results for Metropolis-type chains associated with certain weights on boxes and other subsets of $\mathbb{Z}^{d}$.

In Diaconis and Miclo (2015), a very basic example of this sort is discussed, lazy simple random walk on $\{0,1 \ldots, N\}$ with absorption at 0 and reflection at $N$. This served as a starting point for the present work. Even for such a simple example, the techniques developed below provide improved estimates.

The present approach utilizes powerful tools: Harnack, Poincaré and Nash inequalities. It leads to good results even for domains whose boundaries are quite rugged, namely, inner-uniform domains and John domains. The notions of "Harnack inequality" and "John domain" are quite unfamiliar in the context of finite Markov chains and their installment in this context is non-trivial and interesting mostly when a quantitative viewpoint is implemented carefully. The main contribution of this work is to provide such an implementation.

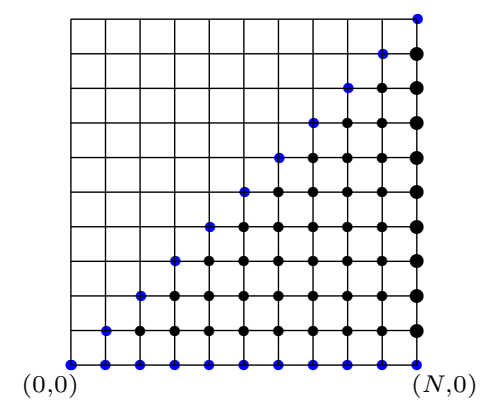

FiguRE 1.1. The forty-five degree finite cone in $\mathbb{Z}^{2}$

The type of finite Markov chains - more precisely, the type of families of finite Markov chains - to which these methods apply is, depending of one's perspective, both quite general and rather restrictive. First, we will mostly deal with reversible Markov chains. Second, the most technical part of this work applies only to families of finite Markov chains whose state spaces have a common "finite-dimensional" nature. Our basic geometric assumptions require that all Markov chains in the general family under consideration have, roughly speaking, the same dimension. The model examples are families of finite Markov chains whose state spaces are subsets of $\mathbb{Z}^{d}$ for some fixed $d$, such as the family of forty-five degree cones parametrized by $N$ shown in Figure 1.1. Many interesting families of finite Markov chains evolve on state spaces that have an "infinite-dimensional nature," e.g., the hypercube $\{0,1\}^{d}$ or the symmetric group $\mathbb{S}_{d}$ where $d$ grows to infinity. Our main results do not 
apply well to these "infinite-dimensional" families of Markov chains, although some intermediary considerations explained in this paper do apply to such examples. See Section 7.1.

The simple example depicted in Figure 1.1 illustrates the aim of this work. Start with simple random walk on the square grid in the plane. For each integer $N>2$, consider the subgraph of the square grid consisting of those vertices $(p, q)$ such that

$$
q<p \leq N \text { and } 0<q<N,
$$

which are depicted by black dots on Figure 1.1. Call this set of vertices $U=U_{N}$. The boundary where the chain is killed (depicted in blue) consists of the bottom and diagonal sides of the cone, i.e., the vertices with either $q=0$ or $p=q$ for $0 \leq p, q \leq N$. Call this set $\partial U=\partial U_{N}$ and set $\mathfrak{X}=\mathfrak{X}_{N}=U_{N} \cup \partial U_{N}$. The vertices along the right side of the cone, $\{(N, q), 1 \leq q<N\}$, have one less neighboring vertex, so we add a loop at each of these vertices. (In Figure 1.1, these vertices are depicted with larger black dots and the loops are omitted for simplicity.)

We are interested in understanding the behavior of the simple random walk on $\mathfrak{X}$ killed at the boundary $\partial U$, before its random killing time $\tau_{U}$. In particular, we would like to have good approximations of quantities such as

$$
\mathbf{P}_{x}\left(\tau_{U}>\ell\right), \quad \mathbf{P}_{x}\left(X_{t}=y \mid \tau_{U}>\ell\right), \quad \mathbf{P}_{x}\left(X_{t}=y \text { and } \tau_{U}>\ell\right)
$$

for $x, y \in U, 0 \leq t \leq \ell$, and

$$
\lim _{\ell \rightarrow \infty} \mathbf{P}_{x}\left(X_{t}=y \mid \tau_{U}>\ell\right)
$$

for $x, y \in U, 0 \leq t<+\infty$ where the time parameter $t$ is integer valued. This limit, if it exists, can be interpreted as the iterated transition probability at time $t$ for the chain conditioned to never be absorbed. We chose the example in Figure 1.1 because it is a rather simple domain, but already demonstrates some of the complexities in approximating the above quantities.

1.2. The Doob-transform technique. Before looking at this example in detail, consider a general irreducible aperiodic Markov kernel $K$ on a finite or countable state space $\mathfrak{X}$. Let $U$ be a finite subset of $\mathfrak{X}$ such that the kernel $K_{U}(x, y)=$ $K(x, y) \mathbf{1}_{U}(x) \mathbf{1}_{U}(y)$ is still irreducible and aperiodic. Let $\left(X_{t}\right)$ be the (discrete time) random walk on $\mathfrak{X}$ driven by $K$, and let $\tau_{U}$ be the stopping time equal to the time of the first exit from $U$ as above.

A rather general result explained in Section 7 implies that the limit

$$
\lim _{\ell \rightarrow \infty} \mathbf{P}_{x}\left(X_{t}=y \mid \tau_{U}>\ell\right), \quad x, y \in U, \quad t \in \mathbb{N}_{\geq 0}
$$

exists and so we can define $K_{\text {Doob }}^{t}(x, y)$ for any $x, y \in U$ and $t \in \mathbb{N}_{\geq 0}$ as

$$
K_{\text {Doob }}^{t}(x, y)=\lim _{\ell \rightarrow \infty} \mathbf{P}_{x}\left(X_{t}=y \mid \tau_{U}>\ell\right)
$$

It is not immediately clear that this collection of $t$-dependent kernels,

$$
K_{\text {Doob }}^{1}, K_{\text {Doob }}^{2}, K_{\text {Doob }}^{3}, \ldots,
$$

has special properties, but it turns out that it is nothing other than the collection of the iterated kernels of the kernel $K_{\text {Doob }}=K_{\text {Doob }}^{1}$ itself, i.e.,

$$
K_{\text {Doob }}^{t}(x, y)=\sum_{z \in U} K_{\text {Doob }}^{t-1}(x, z) K_{\text {Doob }}(z, y) .
$$

Moreover, $K_{\text {Doob }}$ is an irreducible aperiodic Markov kernel. 
To see why this is true, let us explicitly find the kernel $K_{\text {Doob }}$. Recall that, by the Perron-Frobenius theorem, the irreducible, aperiodic, non-negative kernel $K_{U}$ has a real eigenvalue $\beta_{0} \in[0,1]$ which is simple and such that $|\beta|<\beta_{0}$ for every other eigenvalue $\beta$. This top eigenvalue $\beta_{0}$ has a right eigenfunction $\phi_{0}$ and a left eigenfunction $\phi_{0}^{*}$ which are both positive functions on $U$. Set

$$
K_{\phi_{0}}(x, y)=\beta_{0}^{-1} \phi_{0}(x)^{-1} K_{U}(x, y) \phi_{0}(y)
$$

and observe that this is an irreducible aperiodic Markov kernel with invariant probability measure proportional to $\phi_{0}^{*} \phi_{0}$. These facts all follow from the definition and elementary algebra.

In Section 7, we show that

$$
\lim _{\ell \rightarrow \infty} \mathbf{P}_{x}\left(X_{t}=y \mid \tau_{U}>\ell\right)=K_{\phi_{0}}^{t}(x, y),
$$

and hence

This immediately implies that

$$
K_{\text {Doob }}^{t}(x, y)=K_{\phi_{0}}^{t}(x, y)
$$

$$
\mathbf{P}_{x}\left(X_{t}=y \text { and } \tau_{U}>t\right)=K_{U}^{t}(x, y)=\beta_{0}^{t} K_{\text {Doob }}^{t}(x, y) \phi_{0}(x) \phi_{0}(y)^{-1} .
$$

If we assume - this is a big and often unrealistic assumption - that we know the eigenfunction $\phi_{0}$, either via an explicit formula or via "good two-sided estimates," then any question about

$$
\mathbf{P}_{x}\left(X_{t}=y \text { and } \tau_{U}>t\right) \text { or, equivalently, } K_{U}^{t}(x, y)
$$

can be answered by studying

$$
K_{\text {Doob }}^{t}(x, y)
$$

and vice-versa. The key point of this technique is that $K_{\text {Doob }}$ is an irreducible aperiodic Markov kernel with invariant measure proportional to $\phi_{0}^{*} \phi_{0}$ and its ergodic properties can be investigated using a wide variety of classical tools.

The notation $K_{\text {Doob }}$ refers to the fact that this well-established circle of ideas is known as the Doob-transform technique. From now on, we will use the name $K_{\phi_{0}}$ instead, to remind the reader about the key role of the eigenfunction $\phi_{0}$.

1.3. The 45 degree finite discrete cone. In our specific example depicted in Figure $1.1, K_{U}$ is symmetric in $x, y$ so that $\phi_{0}^{*}=\phi_{0}$. We let $\pi_{U} \equiv 2 /(N(N-1))$ denote the uniform measure on $U$ and normalize $\phi_{0}$ by the natural condition $\pi_{U}\left(\phi_{0}^{2}\right)=1$. Then, $\pi_{\phi_{0}}=\phi_{0}^{2} \pi_{U}$ is the invariant probability measure of $K_{\phi_{0}}$ and this pair $\left(K_{\phi_{0}}, \pi_{\phi_{0}}\right)$ is irreducible, aperiodic, and reversible. By applying known quantitative methods to this particular aperiodic, irreducible, ergodic Markov chain, we can approximate the quantities (1.1) and (1.2) as follows.

For any $x=(p, q) \in U$ and any $t$, set $x_{\sqrt{t}}=\left(p_{\sqrt{t}}, q_{\sqrt{t}}\right)$ where

$$
p_{\sqrt{t}}=(p+2\lfloor\sqrt{t / 4}\rfloor) \wedge N \text { and } q_{\sqrt{t}}=(q+\lfloor\sqrt{t / 4}\rfloor) \wedge(N / 2) .
$$

The transformation $x=(p, q) \mapsto x_{\sqrt{t}}=\left(p_{\sqrt{t}}, q_{\sqrt{t}}\right)$ takes any vertex $x=(p, q)$ and pushes it inside $U$ and away from the boundary at scale $\sqrt{t}$ (at least as long as $t \leq N)$. The two key properties of $x_{\sqrt{t}}$ are that it is at distance at most $\sqrt{t}$ from $x$ and at a distance from the boundary $\partial U$ of order at least $\sqrt{t} \wedge N$.

The following six statements can be proven using the techniques in this paper. The first five of these statements generalize to a large class of examples that will be described in detail. The last statement takes advantage of the particular structure 
of the example in Figure 1.1. Note that the constants $c, C$ may change from line to line but are independent of $N, t$ and $x, y \in U=U_{N}$.

(1) For all $N, c N^{-2} \leq 1-\beta_{0} \leq C N^{-2}$. This eigenvalue estimate gives a basic rate at which mass disappears from $U$. (See Theorem 7.23.) For a more precise statement, see item 5 below.

(2) All eigenvalues of $K_{U}$ are real, the smallest one, $\beta_{\min }$, satisfies

$$
\beta_{0}+\beta_{\min } \geq c \beta_{0} N^{-2}
$$

and, for any eigenvalue $\beta$ other than $\beta_{0}$,

$$
\beta_{0}-\beta \geq c \beta_{0} N^{-2} \text {. }
$$

The first of these inequalities shows that $\frac{\beta_{\min }}{\beta_{0}}$, the smallest eigenvalue of $K_{\phi_{0}}$, is strictly larger than -1 , which implies the aperiodicity of $K_{\phi_{0}}$.

(3) For all $x, y, t, N$ with $t \geq N^{2}$

$$
\max _{x, y}\left\{\left|\frac{N(N-1) \mathbf{P}_{x}\left(X_{t}=y \text { and } \tau_{U}>t\right)}{2 \beta_{0}^{t} \phi_{0}(x) \phi_{0}(y)}-1\right|\right\} \leq C e^{-c t / N^{2}} .
$$

A simple interpretation of this (and the following) statement is that

$$
\mathbf{P}_{x}\left(X_{t}=y \text { and } \tau_{U}>t\right)\left(\operatorname{resp} . \mathbf{P}_{x}\left(\tau_{U}>t\right)\right)
$$

is asymptotic to a known function expressed in terms of $\beta_{0}$ and $\phi_{0}$. (See Theorem 7.17.)

(4) For all $x, t, N$ with $t \geq N^{2}$,

$$
\max _{x}\left\{\left|\frac{N(N-1) \mathbf{P}_{x}\left(\tau_{U}>t\right)}{2 \beta_{0}^{t} \phi_{0}(x) \pi_{U}\left(\phi_{0}\right)}-1\right|\right\} \leq C e^{-c t / N^{2}} .
$$

(5) For all $x, t, N$,

$$
c \beta_{0}^{t} \frac{\phi_{0}(x)}{\phi_{0}\left(x_{\sqrt{t}}\right)} \leq \mathbf{P}_{x}\left(\tau_{U}>t\right) \leq C \beta_{0}^{t} \frac{\phi_{0}(x)}{\phi_{0}\left(x_{\sqrt{t}}\right)} .
$$

Unlike the third and fourth statements on this list, which give asymptotic expressions for

$$
\mathbf{P}_{x}\left(X_{t}=y \text { and } \tau_{U}>t\right) \text { and } \mathbf{P}_{x}\left(\tau_{U}>t\right)
$$

for times greater than $N^{2}$, the fifth statement provides a two-sided bound of the survival probability $\mathbf{P}_{x}\left(\tau_{U}>t\right)$ that holds true uniformly for every starting point $x$ and time $t>0$. (See Corollary 8.24.)

(6) For all $N$ and $x=(p, q) \in U$, where $U$ is described in Figure 1.1,

$$
c p q(p+q)(p-q) N^{-4} \leq \phi_{0}(x) \leq C p q(p+q)(p-q) N^{-4} .
$$

Observe that this detailed description of the somewhat subtle behavior of $\phi_{0}$ in all of $U$, together with the previous estimate of $\mathbf{P}_{x}\left(\tau_{U}>t\right)$, provides precise information for the survival probability of the process $\left(X_{t}\right)_{t>0}$ started at any given point in $U$.

In general, it is hard to get detailed estimates on $\phi_{0}$, although some non-trivial and useful properties of $\phi_{0}$ can be derived for large classes of examples. Even in the example given in Figure 1.1, the behavior of $\phi_{0}$ is not easily explained. In this case, it is actually possible to explicitly compute $\phi_{0}$ :

$$
\phi_{0}(x)=4 \kappa_{N} \sin \frac{\pi p}{2 N+1} \sin \frac{\pi q}{2 N+1}\left(\sin ^{2} \frac{\pi p}{2 N+1}-\sin ^{2} \frac{\pi q}{2 N+1}\right) .
$$


The constant $\kappa_{N}$ which makes this eigenfunction have $L^{2}\left(\pi_{U}\right)$-norm equal to 1 can be computed to be $\kappa_{N}=\frac{\sqrt{8 N(N-1)}}{2 N+1}$. The eigenvalue $\beta_{0}$ is

$$
\beta_{0}=\frac{1}{2}\left(\cos \frac{\pi}{2 N+1}+\cos \frac{3 \pi}{2 N+1}\right) .
$$

1.4. A short guide. Because some of the key techniques in this paper have a geometric flavor, we have chosen to emphasize the fact that all our examples are subordinate to some preexisting geometric structure. This underlying geometric structure introduces some of the key parameters that must remain fixed (or appropriately bounded) in order to obtain families of examples to which the results we seek to obtain apply uniformly.

For a flowchart describing the overall organization of this paper, see Figure 1.3.

Generally, we use the language of graphs, and the most basic example of such a structure is a $d$-dimensional square grid. Throughout, the underlying space is denoted by $\mathfrak{X}$. It is finite or countable and its elements are called vertices. It is equipped with an edge set $\mathfrak{E}$ which is a set of pairs undirected $\{x, y\}$ of distinct vertices (note that this excludes loops). Vertices in such pairs are called neighbors. For each $x \in \mathfrak{X}$, the number of pairs in $\mathfrak{E}$ that contain $x$ is supposed to be finite, i.e., the graph is locally finite. The structure $(\mathfrak{X}, \mathfrak{E})$ yields a natural notion of a discrete path joining two vertices and we assume that any two points in $\mathfrak{X}$ can indeed be joined by such a path.

Two rather subtle types of finite subsets of $\mathfrak{X}$ play a key role in this work: $\alpha$-John domains and $\alpha$-inner-uniform domains. Inner-uniform domains are always John domains, but John domains are not always inner-uniform. The number $\alpha \in$ $(0,1]$ is a geometric parameter, and we will mostly consider families of subsets which are all either $\alpha$-John or $\alpha$-inner-uniform for one fixed $\alpha>0$. John domains, named after Fritz John, are discussed in Section 2.1 whereas the discussion of inner-uniform domains is postponed until Section 8. Our most complete results are for inner-uniform domains. These notions are well known in the context of (continuous) Euclidean domains, in particular in the field of conformal and quasiconformal geometry. We provide a discrete version. See Figures 2.6, 8.16, and 8.18 for simple examples.

Whitney coverings are a key tool used in proofs about John and inner-uniform domains. These are collections of inner balls within some domain that are nearly disjoint and have a radius that is proportional to the distance of the center to the boundary. These collections of balls are not themselves a covering of the domain, but the balls with tripled radius are, i.e., they generate a covering. See Section 2.2 for the formal definition and Figure 2.8 for an example. Whitney coverings are absolutely essential to the analysis presented in this paper. For instance, a Whitney covering of a given finite John domain $U$ is used to obtain good estimates for the second largest eigenvalue of a Markov chain (e.g., simple random walk on our graph) forced to remained in the finite domain $U$. See, e.g., Theorem 6.4.

With the geometric graph structure of Section 2 fixed, we add vertex weights, $\pi(x)$ for each $x \in \mathfrak{X}$, and (positive) edge weights, $\mu_{x y}$ for each $\{x, y\} \in \mathfrak{E}$, with the requirement that $\mu$ is subordinated to $\pi$, i.e., $\sum_{y \in \mathfrak{X}} \mu_{x y} \leq \pi(x)$ (often, $\mu_{x y}$ is extended to all pairs by setting $\mu_{x y}=0$ when $\{x, y\} \notin \mathfrak{E}$ ). Section 3.1 explains how each choice of such weights defines a Markov chain and Dirichlet form adapted to 

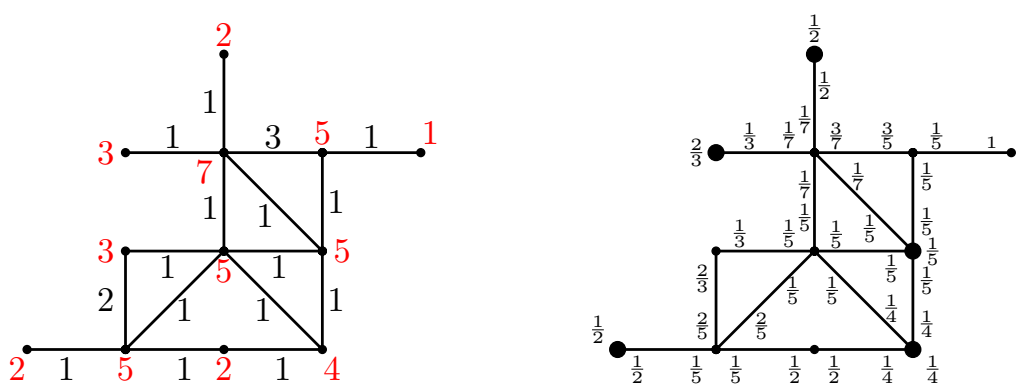

FIGURE 1.2. A graph with weights $\pi, \mu$ ( $\mu$ subordinated to $\pi$ ) and the resulting Markov kernel (with invariant measure $\pi$ ). On the right, each edge $\{x, y\}$ carries two numbers, $K(x, y)$ and $K(y, x)$, with $K(x, y)$ written next to $x$. Large dots indicate non-zero holding and the holding value is indicated nearby.

the geometric structure $(\mathfrak{X}, \mathfrak{E})$. This is illustrated in Figure 1.2 where the Markov kernel $K=K_{\mu}$ is obtained by seting $K(x, y)=\mu_{x y} / \pi(x)$ for $x \neq y$ and $K(x, x)=$ $1-\sum_{y \in \mathfrak{X}} \mu_{x y} / \pi(x)$. We will generally refer to the geometric structure of $(\mathfrak{X}, \mathfrak{E})$ with weights $(\pi, \mu)$ instead of the Markov chain.

Section 3 introduces the important known concepts of volume doubling, moderate growth, various Poincaré inequalities, and Nash inequalities. These notions depend on the underlying structure $(\mathfrak{X}, \mathfrak{E})$ and the weights $(\pi, \mu)$. There is a very large literature on volume doubling, Poincaré inequalities and Nash inequalities in the context of harmonic analysis, global analysis and partial differential equations (see, e.g., Grigor'yan, 2009; Saloff-Coste, 2002 and the references therein for pointers to the literature) and analysis on countable graphs (see Barlow, 2017; Coulhon, 2000; Grigor'yan, 2018; Saloff-Coste, 1997). The notion of moderate growth is from Diaconis and Saloff-Coste $(1994,1996)$ which also cover volume doubling and Poincaré and Nash inequalities in the context of finite Markov chains.

Section 4 is one of the key technical sections of the article. Given an underlying structure $(\mathfrak{X}, \mathfrak{E}, \pi, \mu)$ which satisfies two basic assumptions - volume doubling and the ball Poincaré inequality — we prove a uniform Poincaré inequality for finite $\alpha$ John domains with a fixed $\alpha$. This relies heavily on the definition of a John domain and the use of Whitney coverings. Theorems 4.6 and 4.10 are the main statements in this section. Section 5 provides an extension of the results of Section 4, namely, Theorems 5.5 and 5.11, by modifying the weights on the graph in a way. The line of reasoning for these results is adapted from Jerison (1986); Maheux and SaloffCoste (1995); Saloff-Coste (2002) where earlier relevant references can be found (all these references treat PDE type situations).

Section 6 illustrates the results of Section 5 in the classical context of the Metropolis-Hastings algorithm. Specifically, given a finite John domain $U$ in a graph $(\mathfrak{X}, \mathfrak{E})$, we can modify a simple random walk via edges weights in order to target a given probability distribution. Under certain hypotheses on the target distribution, Section 5 provides useful tools to study the convergence of such chains. We describe several examples in detail. 
Section 7 deals with applications to absorbing Markov chains (or, equivalently for our purpose, chains killed at the boundary). We call such a chain Dirichlet-type by reference to the classical concept of Dirichlet boundary condition. The section has two subsections. The first provides a very general discussion of the Doob transform technique for finite Markov chains. The second applies the results of Section 5 to Dirichlet-type chains in John domains. The main results are Theorems 7.14, 7.17, and 7.23 .

Section 8 introduces the notion of inner-uniform domain in the context of our underlying discrete space $(\mathfrak{X}, \mathfrak{E})$. Theorem 8.9 captures a key property of the PerronFrobenius eigenfunction $\phi_{0}$ in a finite inner-uniform domain. This key property is known as a Carleson estimate after Lennart Carleson. There is a vast literature regarding this estimate and its relation to the boundary Harnack principle in the context of potential theory in Euclidean domains (see, e.g., Ancona, 1978; Bañuelos et al., 1991; Aikawa, 2001, 2005, 2008 and the references and pointers given therein).

Corollary 8.12 is based on the Carleson estimate of Theorem 8.9 and on Theorem 7.14. It provides a (sharp) quantitative convergence (to stationarity) for Doobtransform chains in finite inner-uniform domains. Section 8.2 provides a proof of the Carleson estimate via transfer to the associated cable-process and Dirichlet form. Because the Carleson estimate is a deep and difficult result, it is nice to be able to obtain it from already known results. We use here a similar (and much more general) version of the Carleson estimate in the context of local Dirichlet spaces developed in Lierl and Saloff-Coste (2014b,a) following Aikawa (2001, 2015) and Gyrya and Saloff-Coste (2011). We apply to the eigenfunction $\phi_{0}$ the technique of passage from the discrete graph to the continuous cable space. This requires an interesting argument. (See Proposition 8.18.) Section 8.3 provides more refined results regarding the iterated kernels $K_{U}^{t}$ (chain killed at the boundary) and $K_{\phi_{0}}^{t}$ (associated Doob-transform chain) in the form of two-sided bounds valid at all times and all space location in $U$. A key result is Corollary 8.24 which gives, for inner-uniform domains, a sharp two-sided bound on $\mathbf{P}_{x}\left(\tau_{U}>t\right)$, the probability that the process $\left(X_{t}\right)_{t>0}$ started at $x$ has not yet exited $U$ at time $t$.

The final section, Section 9, describes several explicit examples in detail.

\section{John domains and Whitney coverings}

This section is concerned with notions of a purely geometric nature. Our basic underlying structure can be described as a finite or countable set $\mathfrak{X}$ (vertex set) equipped with an edge set $\mathfrak{E}$ which, by definition, is a set of pairs of distinct vertices $\{x, y\} \subset \mathfrak{X}$. We write $x \sim y$ whenever $\{x, y\} \in \mathfrak{E}$ and say that these two points are neighbors. By definition, a path is a finite sequence of points $\gamma=\left(x_{0}, \ldots, x_{m}\right)$ such that $\left\{x_{i}, x_{i+1}\right\} \in \mathfrak{E}, 0 \leq i<m$. We will always assume that $\mathfrak{X}$ is connected in the sense that, for any two points in $\mathfrak{X}$, there exists a finite path between them. The graph-distance function $d$ assigns to any two points $x, y$ in $\mathfrak{X}$ the minimal length of a path connecting $x$ to $y$, namely,

$$
d(x, y)=\inf \left\{m: \exists \gamma=\left(x_{i}\right)_{0}^{m}, x_{0}=x, x_{m}=y,\left\{x_{i}, x_{i+1}\right\} \in \mathfrak{E}\right\} .
$$

We set

$$
B(x, r)=\{y: d(x, y) \leq r) .
$$




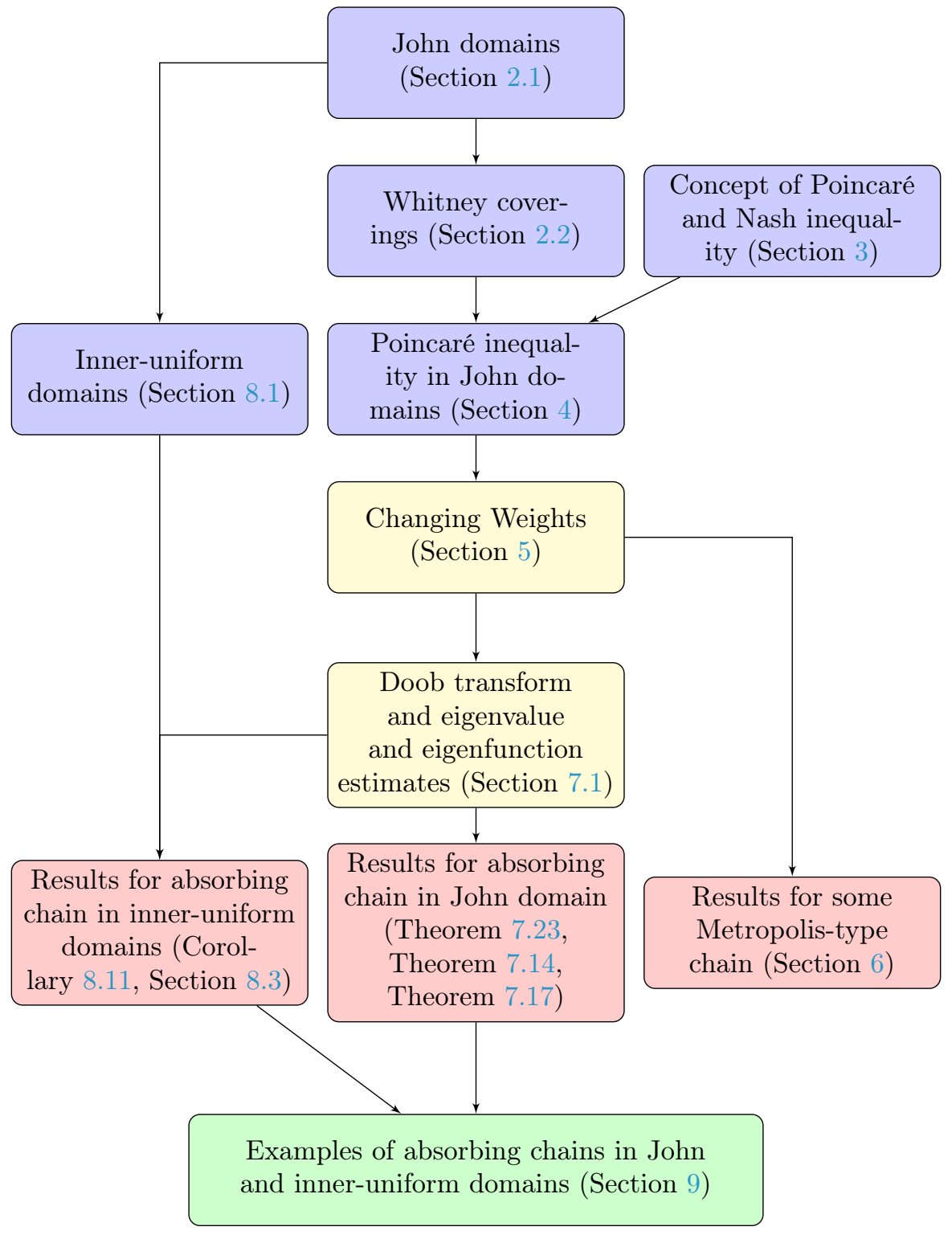

FiguRE 1.3. A flowchart of the main ideas in this paper. The purple boxes denote the background ideas, the yellow boxes denote the main technical sections, the red boxes denote the main results, and the green box denotes the examples.

This is the (closed) metric ball associated with the distance $d$. Note that the radius is a nonnegative real number and $B(x, r)=\{x\}$ for $r \in[0,1)$.

Notation. Given a ball $E=B(x, r)$ with specified center and radius and $\kappa>0$, let $\kappa E$ denote the ball $\kappa E=B(x, \kappa r)$. 
Remark 2.1. We think of $\mathfrak{E}$ as producing a "geometric structure" on $\mathfrak{X}$. Note that loops are not allowed since the elements of $\mathfrak{E}$ are pairs, i.e., subsets of $\mathfrak{X}$ containing two distinct elements. This does not mean that the Markov chains we will consider are forbidden to have positive holding probability at some vertices. The example in the introduction, Figure 1.1, does have positive holding at some vertices (specifically, at $(N, q)$ for $1 \leq q \leq N)$ so the associated Markov chain is allowed to have loops even though the geometric structure does not.

Let $U$ be a subset of $\mathfrak{X}$. Define the boundary of $U$ to be

$$
\partial U=\{y \in \mathfrak{X} \backslash U: \exists x \in U \text { such that }\{x, y\} \in \mathfrak{E}\} .
$$

Note that this is the outer boundary of $U$ in the sense that it sits outside of $U$. We say that $U$ is connected if, for any two points $x, y$ in $U$, the exists a finite path $\gamma_{x y}=\left(x_{0}, x_{1}, \ldots, x_{m}\right)$ with $x_{0}=x$ and $x_{m}=y$ such that $x_{i} \in U$ for $0 \leq i \leq m$. A domain $U$ is a connected subset of $\mathfrak{X}$. We will be interested here in finite domains.

Definition 2.2. Given a domain $U \subseteq \mathfrak{X}$, define the intrinsic distance $d_{U}$ by setting, for any $x, y \in U$,

$d_{U}(x, y)=\inf \left\{m: \exists\left(x_{i}\right)_{0}^{m}, x_{0}=x, x_{m}=y,\left\{x_{i}, x_{i+1}\right\} \in \mathfrak{E}, x_{i} \in U, 0 \leq i<m\right\}$.

In words, $d_{U}(x, y)$ is the graph distance between $x$ and $y$ in the subgraph $\left(U, \mathfrak{E}_{U}\right)$ where $\mathfrak{E}_{U}=\mathfrak{E} \cap(U \times U)$. It is also sometimes called the inner distance (in $\left.U\right)$. Let

$$
B_{U}(x, r)=\left\{y \in U: d_{U}(x, y) \leq r\right\}
$$

be the (closed) ball of radius $r$ around $x$ for the intrinsic distance $d_{U}$.

In the example of Figure 1.1, we set

$$
\mathfrak{X}=\mathfrak{X}_{N}=\{(p, q): 0 \leq q \leq p \leq N\} .
$$

The edge set $\mathfrak{E}=\mathfrak{E}_{N}$ is inherited from the square grid and

$$
U=U_{N}=\{(p, q): 0<q<p \leq N\} .
$$

It follows that the boundary $\partial U$ of $U$ (in $(\mathfrak{X}, \mathfrak{E}))$ is

$$
\partial U=\partial U_{N}=\{(p, p),(p, 0): 0 \leq p \leq N\} .
$$

2.1. John domains. The following definition introduces a key geometric notion which is well known in the areas of harmonic analysis, geometry, and partial differential equations.

Definition 2.3 (John domain). Given $\alpha, R>0$, we say that a finite domain $U \subseteq \mathfrak{X}$, equipped with a point $o \in U$, is in $J(\mathfrak{X}, \mathfrak{E}, o, \alpha, R)$ if the domain $U$ has the property that for any point $x \in U$ there exists a path $\gamma_{x}=\left(x_{0}, \ldots, x_{m}\right)$ of length $m_{x}$ contained in $U$ such that $x_{0}=x$ and $x_{m}=o$, with

$$
\max _{x \in U}\left\{m_{x}\right\} \leq R \text { and } d\left(x_{i}, \mathfrak{X} \backslash U\right) \geq \alpha(1+i),
$$

for $0 \leq i \leq m_{x}$. When the context makes it clear what underlying structure $(\mathfrak{X}, \mathfrak{E})$ is considered, we write $J(o, \alpha, R)$ for $J(\mathfrak{X}, \mathfrak{E}, o, \alpha, R)$.

We can think of a John domain $U$ as being one where any point $x$ is connected to the central point $o$ by a carrot-shaped region, which is entirely contained within $U$. The $x$ is the pointy end of the carrot and the point $o$ is the center of the round, fat end of the carrot. See Figure 8.15 for an illustration. 
Within the lattice $\mathbb{Z}^{d}$, there are many examples of John domains: the lattice balls, the lattice cubes, and the intersection of Euclidean balls and Euclidean equilateral triangles with the lattice. See also Examples 2.9, 2.10, and 2.11 and Figure 2.6 below. Domains having large parts connected through narrow parts are not John. These examples, however, are much too simple to convey the subtlety and flexibility afforded by this definition.

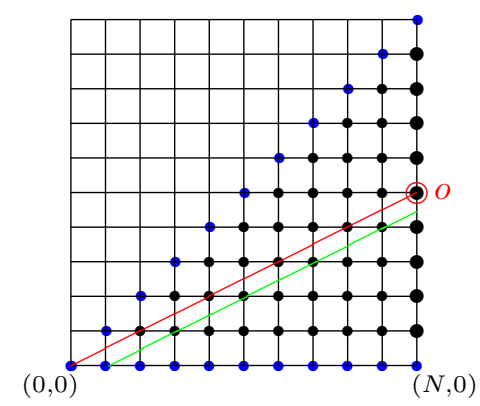

Figure 2.4. The forty-five degree finite cone in $\mathbb{Z}^{2}$ with the "center" marked as a red $o$.

Definition 2.4 ( $\alpha$-John domains). Given $(\mathfrak{X}, \mathfrak{E})$, let $J(\alpha)=J(\mathfrak{X}, \mathfrak{E}, \alpha)$ be the set of all domains $U \subset \mathfrak{X}$ which belong to $J(\mathfrak{X}, \mathfrak{E}, o, \alpha, R)$ for some fixed $o \in U$ and $R>0$. A finite domain in $J(\alpha)$ is called an $\alpha$-John domain.

Definition 2.5 (John center and John radius). For any domain $U \in J(\alpha)$, there is at least one pair $(o, R)$, with $o \in U$ and $R>0$, such that $U \in J(o, \alpha, R)$. Given such a John center $o$, let $R(U, o, \alpha)$ be the smallest $R$ such that $U \in J(o, \alpha, R)$. Assuming $\alpha$ is fixed, we call $R(U, o, \alpha)$ the John-radius of $U$ with respect to $o$.

Remark 2.6. If we apply the second condition of Definition 2.3 to any point in $U$ at distance 1 from the boundary, we see that $\alpha \in(0,1]$.

Remark 2.7. Given $U \in J(\mathfrak{X}, \mathfrak{E}, \alpha)$, define the internal radius of $U$, viewed from $o$, as

$$
\rho_{o}(U)=\max \left\{d_{U}(o, x): x \in U\right\} .
$$

Then, the John-radius $R(U, o, \alpha)$ is always greater than or equal to $\rho_{o}(U)$, i.e., $R(U, o, \alpha) \geq \rho_{o}(U)$. Furthermore, we always have

$$
\min \left\{d_{U}(o, z): z \in \mathfrak{X} \backslash U\right\}=d(o, \mathfrak{X} \backslash U) \geq \alpha(1+R(U, o, \alpha)),
$$

which implies that

$$
\alpha(1+R(U, o, \alpha)) \leq 1+\rho_{o}(U) \leq 1+R(U, o, \alpha)
$$

In words, when $U \in J(\alpha)$ is not a singleton, the John-radius of $U$ and $\rho_{o}(U)$ are comparable, namely,

$$
\frac{\alpha}{2} R(U, o, \alpha) \leq \rho_{o}(U) \leq R(U, o, \alpha) .
$$


We can also compare $\rho_{o}(U)$ to the diameter of the finite metric space $\left(U, d_{U}\right)$. Namely, we have

$$
\rho_{o}(U) \leq \operatorname{diam}\left(U, d_{U}\right) \leq 2 \rho_{o}(U) .
$$

Remark 2.8. Let us compare this definition of a discrete John domain to the continuous version introduced in the classical reference Martio and Sarvas (1979). In Martio and Sarvas (1979), a Euclidean domain $D$ is an $(\alpha, \beta)$-John domain (denoted $D \in \mathbf{J}(\alpha, \beta))$ if there exists a point $o \in D$ such that every $x \in D$ can be joined to $o$ by a rectifiable path $\gamma_{x}:\left[0, T_{x}\right]$ (paramatrized by arc-length) with $\gamma_{x}(0)=x$, $\gamma_{x}\left(T_{x}\right)=o, T_{x} \leq \beta$ and $d_{2}\left(\gamma_{x}(t), \partial D\right) \geq \alpha\left(t / T_{x}\right)$ for $t \in\left[0, T_{x}\right]$. (Here $d_{2}$ is the Euclidean distance.) If one ignores the small modifications made in our definition to account for the discrete graph structure, the class $J(o, \alpha, R)$ is the analogue of the class $\mathbf{J}(\alpha R, R)$ with an explicit center $o$. The smallest $R$ such that $D$ belong to $\mathbf{J}(\alpha R, R)$ with a given center $o$ would be the analogue of our John-radius with respect to $o$.

Example 2.9. Consider the example depicted in Figure 2.4. From the definition of John domain, one can see that it is best to choose $o$ far from the boundary. We pick $o=(N,\lfloor N / 2\rfloor)$, depicted in red in Figure 2.4. For each point $x=(p, q) \in U$ we will define a (graph) geodesic path $\gamma_{x}$ joining $x$ to $o$ in $U$ that satisfies the conditions of a John domain. First, draw two straight lines $L$ and $L^{\prime}$. The first line $L$, shown in red in Figure 2.4, joins $(0,0)$ to $(N, N / 2)$. This is the line with equation $p-2 q=0$ and the integer points on this line are at equal graph-distance from the "boundary lines" $\{(p, q): q=0, p=0,1, \ldots, N\}$ and $\{(p, p): p=0,1, \ldots, N\}$ as shown in blue in Figure 2.4. The line $L^{\prime}$, shown in green, has the equation $p-2 q=1$. For any integer point $x=(p, q)$ on the line $L$, there is graph-geodesic path $\gamma_{x}$ joining $x$ to $o$ obtained by alternatively moving two steps right and one step up. Similarly, for any integer point $x=(p, q)$ on the line $L^{\prime}$, there is a graph-geodesic path $\gamma_{x}$ joining $x$ to $o$ by moving right, then up, to reach a point $x^{\prime}$ on $L$. From there, following $\gamma_{x^{\prime}}$ to $o$. For any integer point $x$ in $U$ above $L$, define $\gamma_{x}$ by moving straight right until reaching an integer point $x^{\prime}$ on $L$, then follow $\gamma_{x^{\prime}}$ to $o$. For those $x \in U$ below $L$, move straight up until reaching an integer point $x^{\prime}$ on $L^{\prime}$. From there, follow the path $\gamma_{x^{\prime}}$ to $o$.

Along any of the paths $\gamma_{x}=\left(x_{0}, \ldots, x_{m}\right)$, with $x_{0}=x \in U$ and $x_{m}=o$, $d\left(x_{i}, \mathfrak{X} \backslash U\right)$ is non-increasing and $d\left(x_{3 i}, \mathfrak{X} \backslash U\right) \geq 1+i$. It follows that $d\left(x_{j}, \mathfrak{X} \backslash U\right) \geq$ $\frac{1}{3}(1+i)$. This proves that $U$ is a John domain with respect to $o$ with parameter $\alpha=1 / 3$ and John-radius $R\left(U, o, \frac{1}{3}\right)=\rho_{o}(U)=N+[N / 2]-3$.

Example 2.10 (Metric balls). Any metric ball $U=B(o, R)$ is a 1-John domain, i.e.,

$$
B(o, R) \in J(\mathfrak{X}, \mathfrak{E}, o, 1, R) .
$$

This is a straightforward but important example. For each $x \in B(o, r)$, fix a path of minimal length $\gamma_{x}=\left(x_{0}=x, x_{1}, \ldots, x_{m_{x}}=o\right), m_{x} \leq R$, joining $x$ to $o$ in $(\mathfrak{X}, \mathfrak{E})$. Then, $d\left(x_{i}, \mathfrak{X} \backslash B(o, R)\right) \geq 1+i$ because, otherwise, there would be a point $z \notin B(o, R)$ and at distance at most $R$ from $o$, contradicting the definition of a ball.

Example 2.11 (Convex sets). In the classical theory of John domains in Euclidean space, convex sets provide basic examples. Round, convex sets have a good John constant ( $\alpha$ close to 1 ) whereas long, narrow ones have a bad John constant ( $\alpha$ close to 0 ). We will describe how this theory applies in the case of discrete convex 
sets, but first, let us review the continuous case. Here is how the definition of Euclidean John domain given in Martio and Sarvas (1979) applies to Euclidean convex sets. A Euclidean convex set $C$ belongs to $\mathbf{J}(\alpha, \beta)$ (see Martio and Sarvas, 1979, Definition 2.1 and Remark 2.8 above) if and only if there exists $o \in C$ such that

$$
B_{2}(o, \alpha) \subset C \subset B_{2}(o, \beta) \text {. }
$$

Here the balls are Euclidean balls and this is indicated by the subscript 2, referencing the $d_{2}$ metric. This condition is obviously necessary for $C \in \mathbf{J}(\alpha, \beta)$. To see that it is sufficient, observe that along the line-segment $\gamma_{x y}$ between any two points $x, y \in C$, parametrized by arc-length and length $T$, the function $f(t)=$ $d_{2}\left(\gamma_{x y}(t), C^{c}\right)$, defined on $[0, T]$, is concave (it is the minimum of the distances to the supporting hyperplanes defining $C)$. Hence, if we assume that $B(y, \alpha) \subset C$, either $d_{2}\left(y, C^{c}\right)<d_{2}\left(x, C^{c}\right)$ and then $d_{2}\left(\gamma_{x y}(t), C^{c}\right) \geq \alpha \geq \alpha \frac{t}{T}$, or $d_{2}\left(y, C^{c}\right) \geq d_{2}\left(x, C^{c}\right)$ and

which gives

$$
d_{2}\left(\gamma_{x y}(t), C^{c}\right)-d_{2}\left(x, C^{c}\right) \geq \frac{t}{T}\left(d_{2}\left(y, C^{c}\right)-d_{2}\left(x, C^{c}\right)\right)
$$

$$
d_{2}\left(\gamma_{x y}(t), C^{c}\right) \geq \alpha \frac{t}{T}+\left(1-\frac{t}{T}\right) d_{2}\left(x, C^{c}\right) \geq \alpha \frac{t}{T} .
$$

To transition to discrete John domains, we first consider the case of finite domains in $\mathbb{Z}^{2}$ because it is quite a bit simpler than the general case (compare Diaconis and Saloff-Coste, 1996, Section 6 and Virág, 1998). In $\mathbb{Z}^{2}$, we can show that any finite sub-domain $U$ of $\mathbb{Z}^{2}$ (this means we assume that $U$ is graph connected) obtained as the trace of a convex set $C$ such that $B_{2}(o, \alpha R) \subseteq C \subseteq B(o, R)$ for some $\alpha \in(0,1)$ and $R>0$ is a $\alpha^{\prime}$-John domain with $\alpha^{\prime}$ depending only on $\alpha$. (For a discussion of convex domains in $\mathbb{Z}^{d}$ with $d>2$, see Appendix A.)

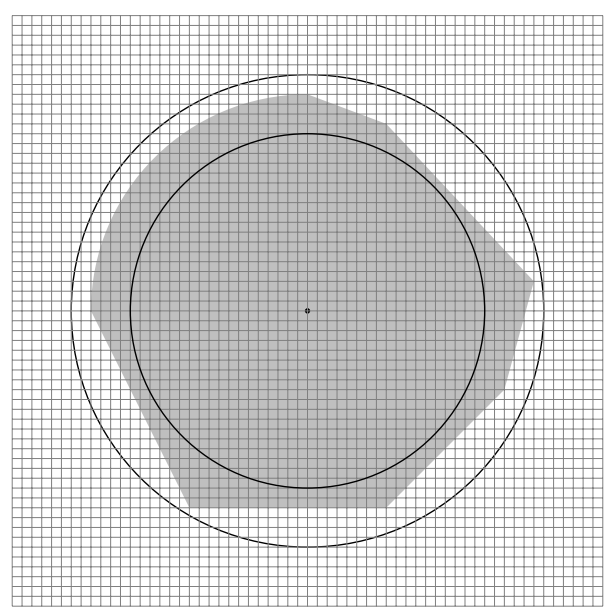

FiguRe 2.5. A finite discrete "convex subset" of $\mathbb{Z}^{2}$

Convexity is certainly not necessary for a family of connected subsets of $\mathbb{Z}^{d}$ to be $\alpha$-John domains with a uniform $\alpha \in(0,1)$. Figure 2.6 gives an example of such a family that is far from convex in any sense. If we denote by $U_{N}$ the set depicted for a given $N$ and let $o_{N}=(\lfloor 2 N / 3\rfloor,\lfloor N / 2\rfloor)$ the chosen central point, then there 
are positive reals $\alpha, c, C$, independent of $N$, such that $U_{N}$ is a $J\left(o_{N}, \alpha, R\right)$ with $c N \leq R \leq C N$. Figure 2.7 gives an example of a family of sets that is NOT uniformly in $J(\alpha)$, for any $\alpha>0$.

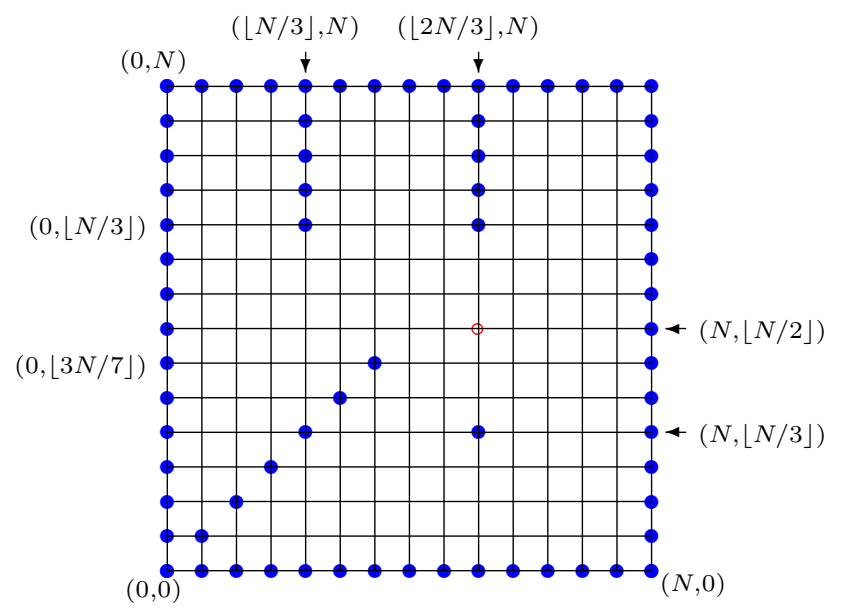

FiguRE 2.6. A non-convex example of John domain, with the boundary points indicated in blue, and center $o$ indicated in red.

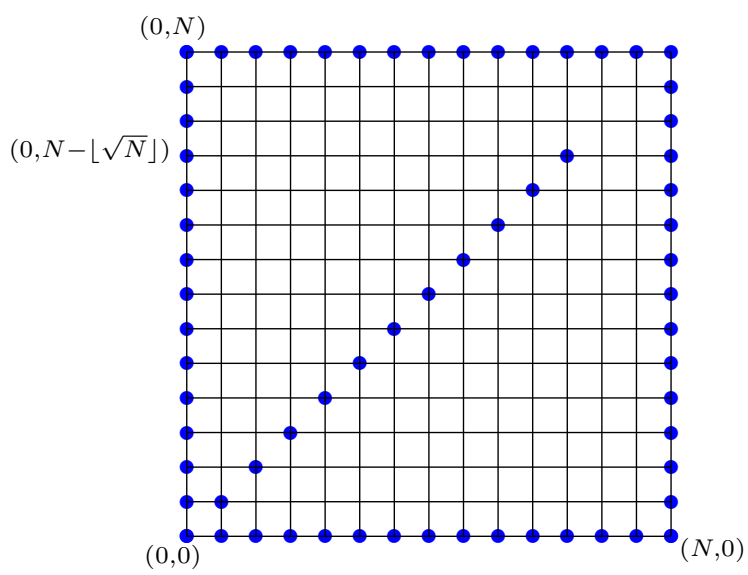

Figure 2.7. A family of subsets that are not uniformly John domains, with the boundary points indicated in blue. The passage between the top and bottom triangles is too narrow.

The following lemma shows that any inner-ball $B_{U}(x, r)$ in a John domain contains a ball from the original graph with roughly the same radius. When the graph 
is equipped with a doubling measure (see Section 3), this shows that the inner balls for the domain $U$ have volume comparable to that of the original balls.

Lemma 2.12. Given $U \in J(\mathfrak{X}, \mathfrak{E}, \alpha)$, recall that $\rho_{o}(U)=\max \left\{d_{U}(o, x): x \in U\right\}$. For any $x \in U$ and $r \in\left[0,2 \rho_{o}(U)\right]$, there exists $x_{r} \in U$ such that $B\left(x_{r}, \alpha r / 8\right) \subset$ $B_{U}(x, r)$. For $r \geq 2 \rho_{o}(U)$, we have $B_{U}(x, r)=U$.

Proof: The statement concerning the case $r \geq 2 \rho_{o}(U)$ is obvious. We consider three cases. First, consider the case when $o \in B_{U}(x, r / 4)$ and $\rho_{o}(U) \leq r<2 \rho_{o}(U)$. Then $B\left(o, \alpha \rho_{o}(U) / 4\right) \subseteq B_{U}(x, r)$ and we can set $x_{r}=o$. Second, assume that $o \in$ $B_{U}(x, r / 4)$ and $r<\rho_{o}(U)$. Recall from Remark 2.7 that $d(o, \mathfrak{X} \backslash U) \geq \alpha\left(1+\rho_{o}(U)\right)$. It follows that $B(o, \alpha r / 8) \subset U$ and $B(o, \alpha r / 8) \subset B_{U}(x, r)$. We can again set $x_{r}=o$. Finally, assume that $o \notin B_{U}(x, r / 4)$. If $r<8$, we can take $x_{r}=x$. When $r \geq 8$, let $\gamma_{x}=\left(z_{0}=x, z_{1}, \ldots, z_{m}=o\right)$ be the John-path from $x$ to $o$ and let $x_{r}=z_{i}$, where $z_{i}$ is the first point on $\gamma_{x}$ such that $z_{i+1} \notin B_{U}(x,\lfloor r / 4\rfloor)$. By construction, we have $d_{U}\left(x, x_{r}\right) \leq\lfloor r / 4\rfloor+1 \leq r / 2, i(x, r) \geq\lfloor r / 4\rfloor$ and

$$
\delta\left(x_{r}\right) \geq \alpha(1+\lfloor r / 4\rfloor) \geq \alpha r / 4,
$$

where $\delta(x)=d(x, \mathfrak{X} \backslash U)$. Therefore $B\left(x_{r}, \alpha r / 8\right) \subset U$ and $B_{U}\left(x_{r}, \alpha r / 8\right)=$ $B\left(x_{r}, \alpha r / 8\right) \subset B_{U}(x, r)$.

2.2. Whitney coverings. Let $U$ be a finite domain in the underlying graph $(\mathfrak{X}, \mathfrak{E})$ (this graph may be finite or countable). Fix a small parameter $\eta \in(0,1)$. For each point $x \in U$, let

$$
B_{x}^{\eta}=\{y \in U: d(x, y) \leq \eta \delta(x) / 4\}
$$

be the ball centered at $x$ of radius $r(x)=\eta \delta(x) / 4$ where

$$
\delta(x)=d(x, \mathfrak{X} \backslash U)
$$

is the distance from $x$ to $\mathfrak{X} \backslash U$, the boundary of $U$ in $(\mathfrak{X}, \mathfrak{E})$. The finite family $\mathcal{F}=\left\{B_{x}^{\eta}: x \in U\right\}$ forms a covering of $U$. Consider the set of all sub-families $\mathcal{V}$ of $\mathcal{F}$ with the property that the balls $B_{x}^{\eta}$ in $\mathcal{V}$ are pairwise disjoint. This is a partially ordered finite set and we pick a maximal element

$$
\mathcal{W}=\left\{B_{x_{i}}^{\eta}: 1 \leq i \leq M\right\}
$$

which, by definition, is a Whitney covering of $U$. Note that the Whitney covering of $U$ is not a covering itself, but it generates a covering, because the triples of the in $\mathcal{W}$, i.e., the balls with tripled radius, are a covering of $U$. Because the balls in $U$ are disjoint, this is a relatively efficient covering.

The size $M$ of this covering will never appear in our computation and is introduced strictly for convenience. This integer $M$ depends on $U, s, \eta$ and on the particular choice made among all maximal elements in $\mathcal{V}$.

Whitney coverings are useful because they allow us to do manipulations on balls that form a covering - such as doubling their size - without leaving the domain $U$. Moreover, for any $k<4 / \eta$, the closed ball $\{y: d(x, y) \leq k r(x)\}$ is entirely contained in $U$.

In the above (standard, discrete) version of the notion of Whitney covering, the largest balls are of size comparable to $\eta \max \{d(x, \mathfrak{X} \backslash U): x \in U\}$. In the following $s$-version, $s \geq 1$, where $s$ is a (scale) parameter, the size of the largest balls are at 


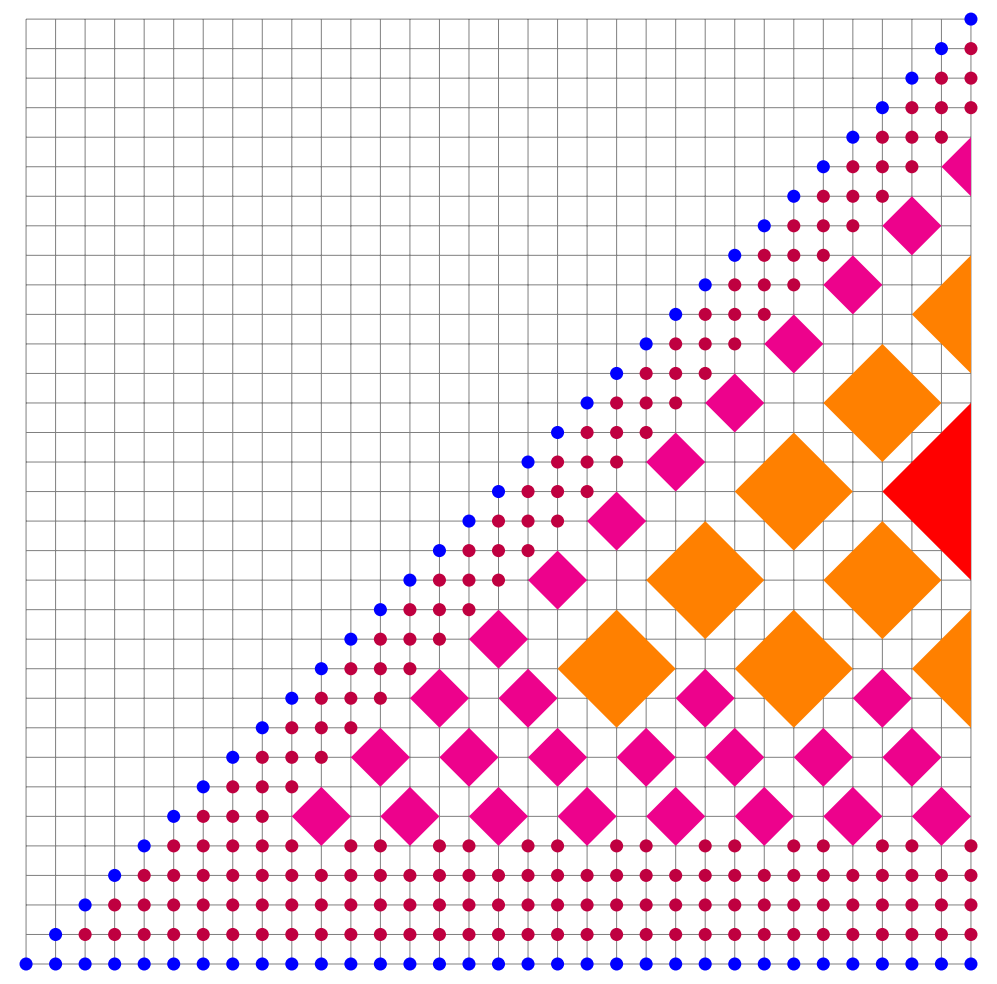

Figure 2.8. A Whitney covering of the forty-five degree cone with $\eta=\frac{4}{5}$, where the boundary of the cone is indicated in blue. The color of each ball in the Whitney covering indicates its radius.

most $s$. Fix $s \geq 1$ and a small parameter $\eta \in(0,1)$ as before. For each point $x \in U$, let

$$
B_{x}^{s, \eta}=\{y: d(x, y) \leq \min \{s, \eta \delta(x) / 4\}\}
$$

be the ball centered at $x$ of radius $r(x)=\min \{s, \eta \delta(x) / 4\}$. Note as before that, for any $k<4 / \eta$, the closed ball $\{y: d(x, y) \leq k \delta(x) / 4\}$ is entirely contained in $U$. The finite family $\mathcal{F}_{s}=\left\{B_{x}^{s, \eta}: x \in U\right\}$ form a covering of $U$. Consider the set of all sub-families $\mathcal{V}_{s}$ of $\mathcal{F}_{s}$ with the property that the balls $B_{x}^{s, \eta}$ in $\mathcal{V}_{s}$ are pairwise disjoint. These subfamilies form a partially ordered finite set and, just as we did with $\mathcal{F}$, we pick a maximal element

$$
\mathcal{W}_{s}=\left\{B_{x_{i}}^{s, \eta}: 1 \leq i \leq M\right\},
$$

which is the $s$-Whitney covering. See Figure 2.9 for an example.

As before, the size $M$ of this covering will never appear in our computations. It will be useful to split the family $\mathcal{W}_{s}$ into its two natural components, $\mathcal{W}_{s}=$ $\mathcal{W}_{=s} \cup \mathcal{W}_{<s}$ where $\mathcal{W}_{=s}$ is the subset of $\mathcal{W}_{s}$ of those balls $B\left(x_{i}, r\left(x_{i}\right)\right)$ such that $r\left(x_{i}\right)=s$.

Remark 2.13. When the domain $U$ is finite (in a more general context, bounded) any Whitney covering $\mathcal{W}_{s}$ with parameter $s$ large enough, namely

$$
s \geq \eta\left(\rho_{o}(U)+1\right) / 4,
$$



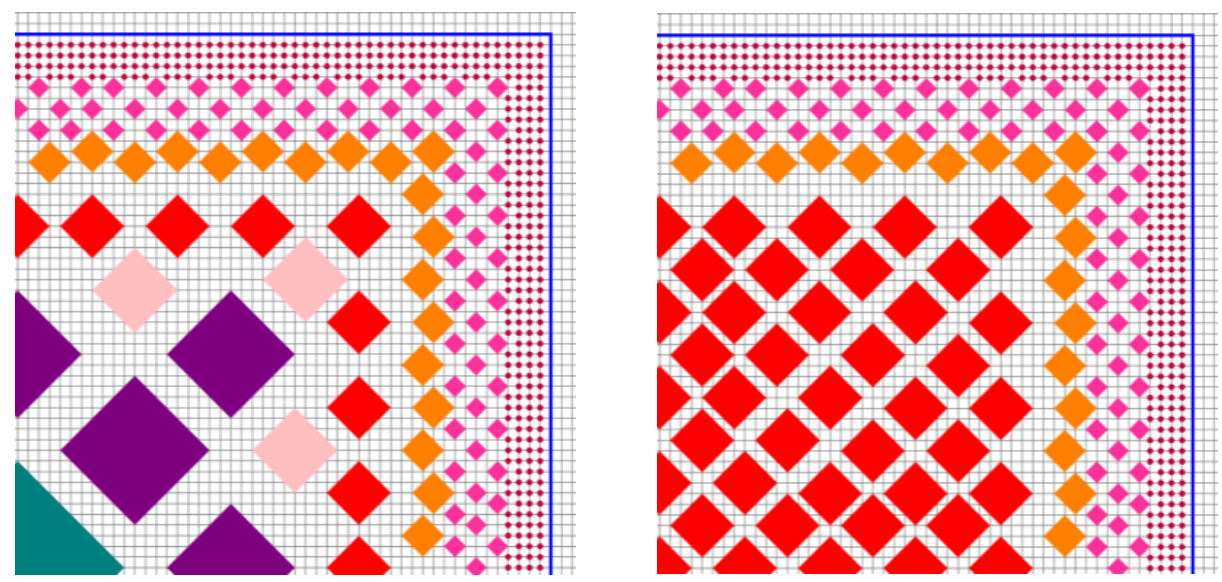

Figure 2.9. The left figure shows a standard Whitney covering of the upper right corner of a square (boundary indicated in blue) with $\eta=\frac{4}{5}$. The right figure shows an $s$-Whitney covering, where $s=3$, i.e., the maximum radius of a Whitney ball is 3 .

is simply a Whitney covering $\mathcal{W}$ because $\min \{s, \eta \delta(x) / 4\}=\eta \delta(x) / 4$ for all $x \in U$. It follows that properties that hold for all $\mathcal{W}_{s}, s>0$, also hold for any standard Whitney coverings $\mathcal{W}$.

Lemma 2.14 (Properties of $\mathcal{W}_{s}, s \geq 1$ ). For any $s>0$, the family $\mathcal{W}_{s}$ has the following properties.

(1) The balls $B_{x_{i}}^{s, \eta}=B\left(x_{i}, r\left(x_{i}\right)\right), 1 \leq i \leq M$, are pairwise disjoint and

$$
U \subseteq \bigcup_{1}^{M} B\left(x_{i}, 3 r\left(x_{i}\right)\right) .
$$

In other words, the balls with tripled radius cover $U$.

(2) For any $\rho \leq 4 / \eta$ and any $z \in B\left(x_{i}, \rho r\left(x_{i}\right)\right)$,

$$
\delta\left(x_{i}\right)(1-\rho \eta / 4) \leq \delta(z) \leq(1+\rho \eta / 4) \delta\left(x_{i}\right)
$$

and

$$
(1-\rho \eta / 4) r\left(x_{i}\right) \leq r(z) \leq(1+\rho \eta / 4) r\left(x_{i}\right) .
$$

(3) For any $\rho \leq 2 / \eta$, if the balls $B\left(x_{i}, \rho r\left(x_{i}\right)\right)$ and $B\left(x_{j}, \rho r\left(x_{j}\right)\right)$ intersect then

$$
\frac{1}{3} \leq \frac{1-\rho \eta / 4}{1+\rho \eta / 4} \leq \frac{\delta\left(x_{i}\right)}{\delta\left(x_{j}\right)} \leq \frac{1+\rho \eta / 4}{1-\rho \eta / 4} \leq 3 .
$$

Proof: We prove the first assertion. Consider a point $z \in U$. Since $\mathcal{W}_{s}$ is maximal, the ball $B_{z}^{s, \eta}$ intersects $\cup_{1}^{M} B\left(x_{i}, r\left(x_{i}\right)\right)$. So there is an $i \in\{1,2, \ldots, M\}$ and a $y \in B_{x_{i}}^{s, \eta}$ such that $y \in B_{z}^{s, \eta}$. By the triangle inequality,

$$
\delta\left(x_{i}\right) \geq \delta(z)-r\left(x_{i}\right)-r(z) \geq \delta(z)-\eta \delta\left(x_{i}\right) / 4-\eta \delta(z) / 4,
$$

which yields,

and hence,

$$
(1+\eta / 4) \delta\left(x_{i}\right) \geq(1-\eta / 4) \delta(z),
$$

$$
(1+\eta / 4) r\left(x_{i}\right) \geq(1-\eta / 4) r(z) .
$$


It follows that

$$
d\left(x_{i}, z\right) \leq r\left(x_{i}\right)+r(z) \leq r\left(x_{i}\right)\left(1+\frac{1+\eta / 4}{1-\eta / 4}\right) \leq\left(1+\frac{5}{3}\right) r\left(x_{i}\right) .
$$

Therefore, $z \in B\left(x_{i}, 3 r\left(x_{i}\right)\right)$.

The proofs of (2)-(3) follow the same line of reasoning.

\section{Poincaré and Nash inequalities}

In this section, we fix a background graph structure $(\mathfrak{X}, \mathfrak{E})$ and use $x \sim y$ to indicate that $\{x, y\} \in \mathfrak{E}$. As before, let $d(x, y)$ denote the graph distance between $x$ and $y$, and let

$$
B(x, r)=\{y: d(x, y) \leq r\}
$$

be the ball of radius $r$ around $x$. (Note that balls are not uniquely defined by their radius and center, i.e., it's possible that $B(x, r)=B(\tilde{x}, \tilde{r})$ for $x \neq \tilde{x}$ and $r \neq \tilde{r}$.) In addition we will assume that $\mathfrak{X}$ is equipped with a measure $\pi$ and, later, that $\mathfrak{E}$ is equipped with an edge weight $\mu=\left(\mu_{x y}\right)$ defining a Dirichlet form.

Definition 3.1 (Doubling). We say that $\pi$ is doubling (with respect to $(\mathfrak{X}, \mathfrak{E})$ ) if there exists a constant $D$ (the doubling constant) such that, for all $x \in \mathfrak{X}$ and $r>0$,

$$
V(x, 2 r) \leq D V(x, r)
$$

Doubling is a critical property of the measure $\pi$ with many important implications. A related notion which we use is that of moderate growth. See Appendix B for more information.

3.1. Edge weights, associated Markov chains and Dirichlet forms. This section introduces symmetric edge weights $\mu_{x y}=\mu_{y x} \geq 0$ and the associated quadratic form

$$
\mathcal{E}_{\mu}(f, g)=\frac{1}{2} \sum_{x, y \in \mathfrak{X}}(f(x)-f(y))(g(x)-g(y)) \mu_{x y} .
$$

Definition 3.2. Consider a set of symmetric edge weights $\mu=\left(\mu_{x y}\right)_{x \neq y \in \mathfrak{X}}$.

(1) We say the edge weight $\mu=\left(\mu_{x y}\right)_{x \neq y \in \mathfrak{X}}$, is adapted to $\mathfrak{E}$ if

$$
\mu_{x y}>0 \text { if and only }\{x, y\} \in \mathfrak{E} .
$$

(2) We say that the edge weight $\mu=\left(\mu_{x y}\right)_{x \neq y \in \mathfrak{X}}$ is elliptic with constant $P_{e} \in(0, \infty)$ with respect to $(\mathfrak{X}, \mathfrak{E}, \pi)$ if

$$
\forall\{x, y\} \in \mathfrak{E}, \quad P_{e} \mu_{x y} \geq \pi(x) .
$$

(3) We say that the edge weight $\mu=\left(\mu_{x y}\right)_{x, y \in \mathfrak{X}}$ is subordinated to $\pi$ on $\mathfrak{X}$ if

$$
\forall x \in \mathfrak{X}, \quad \sum_{y \in \mathfrak{X}} \mu_{x y} \leq \pi(x) .
$$

Remark 3.3. An adapted edge weight $\mu$ is always such that $\mu_{x y}=0$ if $\{x, y\} \notin \mathfrak{E}$, so the definition of adapted edge weight means that $\mu$ is carried by the edge set $\mathfrak{E}$ in a qualitative sense. Ellipticity makes this quantitative in the sense that $\mu_{x y} \geq$ $P_{e}^{-1} \pi(x)$. Note that, with this definition, the smaller the ellipticity constant, the better. 
Remark 3.4. Since $\mu_{x y}=\mu_{y x}$, the ellipticity condition is equivalent to

$$
P_{e} \mu_{x y} \geq \pi(y)
$$

and also to $P_{e} \mu_{x y} \geq \max \{\pi(x), \pi(y)\}$.

The condition $\sum_{y \in \mathfrak{X}} \mu_{x y}<+\infty$ implies immediately that the quadratic form $\mathcal{E}_{\mu}$ defined on finitely supported functions is closable with dense domain in $L^{2}(\pi)$. In that case, the data $(\mathfrak{X}, \pi, \mu)$ defines a continuous time Markov process on the state space $\mathfrak{X}$, reversible with respect to the measure $\pi$. This Markov process is the process associated to the Dirichlet form obtained by closing $\mathcal{E}_{\mu}$ in $L^{2}(\pi)$ and to the associated self-adjoint semigroup $H_{t}$. See, e.g., Fukushima et al. (2011, Example $1.2 .4)$.

Definition 3.5. Assume the the edge weight $\mu$ is subordinated to $\pi$, i.e.,

$$
\forall x \in \mathfrak{X}, \sum_{y \in \mathfrak{X}} \mu_{x y} \leq \pi(x) .
$$

Set

$$
K_{\mu}(x, y)=\left\{\begin{array}{cc}
\mu_{x y} / \pi(x) & \text { for } x \neq y \\
1-\left(\sum_{y \in \mathfrak{X}} \mu_{x y} / \pi(x)\right) & \text { for } x=y
\end{array}\right.
$$

Note that the condition that $\mu$ is subordinated to $\pi$ is necessary and sufficient for the semigroup $H_{t}$ to be of the form $H_{t}=e^{-t(I-K)}$ where $K$ is a Markov kernel on $\mathfrak{X}$. Indeed, we then have $K=K_{\mu}$. This Markov kernel is always reversible with respect to $\pi$. Of course, if we replace the condition $\sum_{y \in \mathfrak{X}} \mu_{x y} \leq \pi(x)$ by the weaker condition $\sum_{y \in \mathfrak{X}} \mu_{x y} \leq A \pi(x)$ for some finite $A$, then $H_{t}=e^{-A t\left(I-K_{A^{-1}}\right)}$ where $A^{-1} \mu$ is the weight $\left(A^{-1} \mu_{x y}\right)_{x, y \in \mathfrak{X}}$.

\subsection{Poincaré inequalities.}

Definition 3.6 (Ball Poincaré Inequality). We say that $(\mathfrak{X}, \mathfrak{E}, \pi, \mu)$ satisfies the ball Poincaré inequality with parameter $\theta$ if there exists a constant $P$ (the Poincaré constant) such that, for all $x \in \mathfrak{X}$ and $r>0$,

$$
\sum_{z \in B(x, r)}\left|f(z)-f_{B}\right|^{2} \pi(z) \leq \operatorname{Pr}^{\theta} \sum_{z, y \in B(x, r), z \sim y}|f(z)-f(y)|^{2} \mu_{z y} .
$$

Remark 3.7. Under the doubling property, ellipticity is somewhat related to the Poincaré inequality on balls of small radius. Whenever the ball of radius 1 around a point $x$ is a star (i.e., there are no neighboring relations between the neighbors of $x$ as, for instance, in a square grid) the ball Poincaré inequality with constant $P$ implies easily that, at such point $x$ and for any $y \sim x$,

$$
\pi(y) \leq P D^{2} \mu_{x y}
$$

To see this, fix $y \in B(x, 1)$ and apply the Poincaré inequality on $B(x, 1)$ to the test function defined on $B(x, 1)$ by

$$
f(x)= \begin{cases}-c & \text { if } x \neq y \\ 1 & \text { if } x=y\end{cases}
$$




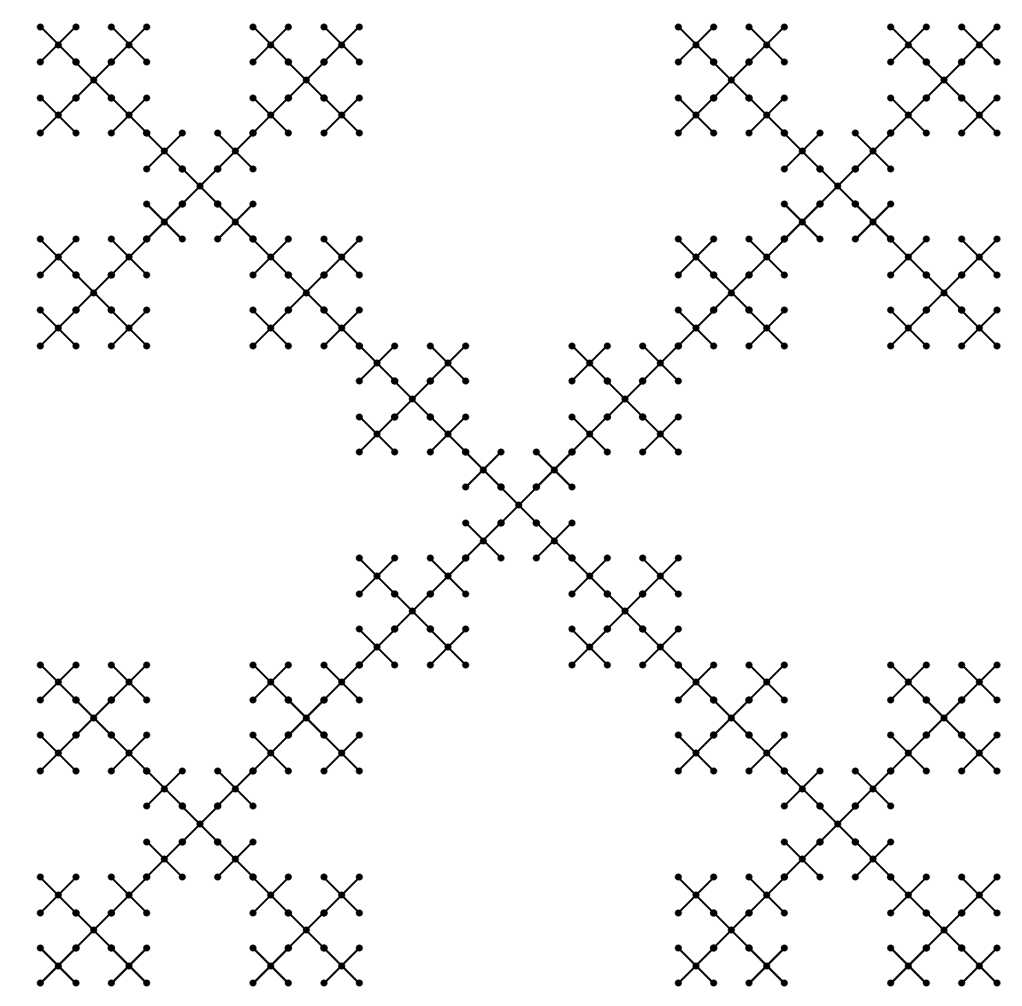

FIGURE 3.10. A finite piece of the Vicsek graph, an infinite graph which is both a tree and a fractal graph, has volume growth of type $r^{d}$ with $d=\log 5 / \log 3$ and satisfies the Poincaré inequality on balls with parameter $\theta=1+d=1+\log 5 / \log 3$.

where $c=\pi(y) /(\pi(B(x, 1))-\pi(y))$ so that the mean of $f$ over $B(x, 1)$ is 0 . Recall that $B(x, 1)$ is assumed to be a star and note that $0 \leq c \leq \pi(y) / \pi(x) \leq D$ where $D \geq 1$ is the doubling constant. This yields

$$
\pi(y) \leq P(1-c)^{2} \mu_{x y} \leq P D^{2} \mu_{x y} .
$$

Hence, when all balls of radius 1 are stars then the ball Poincaré inequality with constant $P$ implies ellipticity with constant $P_{e}=D^{2} P$. (See Remark 3.4.) However, when it is not the case that all balls of radius 1 are stars then the ball Poincaré inequality does not necessarily imply ellipticity.

Definition 3.8 (Classical Poincaré inequality). A finite subset $U$ of $\mathfrak{X}$, equipped with the restrictions of $\pi$ and $\mu$ to $U$ and $\mathfrak{E} \cap(U \times U)$ satisfies the (Neumann-type) Poincaré inequality with constant $P(U)$ if and only if, for any function $f$ defined on $U$,

$$
\sum_{x \in U}\left|f(x)-f_{U}\right|^{2} \pi(x) \leq P(U) \mathcal{E}_{\mu, U}(f, f)
$$


where

$$
\mathcal{E}_{\mu, U}(f, g)=\frac{1}{2} \sum_{x, y \in U}(f(x)-f(y))(g(x)-g(y)) \mu_{x y}
$$

and $f_{U}=\pi(U)^{-1} \sum_{x \in U} f(x) \pi(x)$.

Example 3.9. Assume that $\mathfrak{X}$ is finite and that $(\mathfrak{X}, \mathfrak{E}, \pi, \mu)$ satisfies the ball Poincaré inequality with parameter $\theta$. Then, taking $r=\operatorname{diam}(\mathfrak{X}) \operatorname{implies}$ that $\mathfrak{X}$ satisfies the Poincaré inequality with constant $P(\mathfrak{X})=2 P \operatorname{diam}(\mathfrak{X})^{\theta}$.

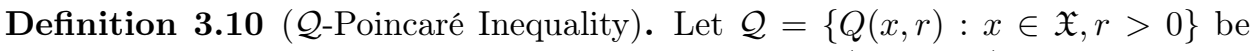
a given collection of finite subsets of $\mathfrak{X}$. We say that $(\mathfrak{X}, \mathfrak{E}, \pi, \mu)$ satisfies the $\mathcal{Q}$ Poincaré inequality with parameter $\theta$ if there exists a constant $P$ such that for any function $f$ with finite support and $r>0$,

$$
\sum_{x \in \mathfrak{X}}\left|f(x)-Q_{r} f(x)\right|^{2} \pi(x) \leq \operatorname{Pr}^{\theta} \mathcal{E}_{\mu}(f, f)
$$

where

$$
\mathcal{E}_{\mu}(f, g)=\frac{1}{2} \sum_{x, y \in \mathfrak{X}}(f(x)-f(y))(g(x)-g(y)) \mu_{x y}
$$

and $Q_{r} f(x)=\pi(Q(x, r))^{-1} \sum_{y \in Q(x, r)} f(y) \pi(y)$.

Example 3.11. The typical example of a collection $\mathcal{Q}$ is the collection of all balls $B(x, r)$. In that case, $Q_{r} f(x)=f_{r}(x)$ is simply the average of $f$ over $B(x, r)$. In this case, the $\mathcal{Q}$-Poincaré inequality is often called a pseudo-Poincaré inequality. Furthermore, if $(\mathfrak{X}, \mathfrak{E}, \pi, \mu)$ satisfies the doubling property and the ball Poincaré inequality then it automatically satisfies the pseudo-Poincaré inequality.

The notion of $\mathcal{Q}$-Poincaré inequality is tailored to make it a useful tool to prove Nash inequalities. (See Proposition 3.12.) An appropriate collection $\mathcal{Q}$ must be determined that satisfies this inequality, but there is no other restriction on what the collection $\mathcal{Q}$ might be. In applications, the set $Q(x, r)$ tends to grow in size with $r$. We can think of $Q_{r} f$ as a regularized version of $f$ at scale $r$. The $\mathcal{Q}$-Poincaré inequality provides control (in $L^{2}$-norm) of the difference $f-Q_{r} f$. If $\mathfrak{X}$ is finite and there is an $R>0$ such that $Q(x, R)=\mathfrak{X}$ for all $x$ then $Q_{R} f(x)$ is the $\pi$ average of $f$ over $\mathfrak{X}$ and the $\mathcal{Q}$-Poincaré inequality at level $R$ becomes a classical Poincaré inequality as defined above.

3.3. Nash inequality. Nash inequalities (in $\mathbb{R}^{n}$ ) were introduced in a famous 1958 paper of John Nash as a tool to capture the basic decay of the heat kernel over time. Later, they where used by many authors for a similar purpose in the contexts of Markov semigroups and Markov chains on countable graphs. Nash inequalities where first used in the context of finite Markov chains in Diaconis and Saloff-Coste (1996), a paper to which we refer for a more detailed introduction.

Assume that $(\mathfrak{X}, \mathfrak{E})$ is equipped with a measure $\pi$ and an edge weight $\mu$. The following is a variant of Diaconis and Saloff-Coste (1996, Theorem 5.2). The proof is the same.

Proposition 3.12. Assume that there is a family of operators defined on finitely supported functions on $\mathfrak{X}, Q_{s}$ (with $0 \leq s \leq T$ ) such that

$$
\left\|Q_{s} f\right\|_{\infty} \leq M(1+s)^{-\nu}\|f\|_{1}
$$


for some $\nu \geq 0$ and that the edge weight $\mu=\left(\mu_{x, y}\right)$ is such that

$$
\left\|f-Q_{s} f\right\|_{2}^{2} \leq P s^{\theta} \mathcal{E}_{\mu}(f, f)
$$

then the Nash inequality

$$
\|f\|_{2}^{2(1+\theta / \nu)} \leq C\left[\mathcal{E}_{\mu}(f, f)+\frac{1}{P T^{\theta}}\|f\|_{2}^{2}\right]\|f\|_{1}^{2 \theta / \nu}
$$

holds with $C=\left(1+\frac{\theta}{2 \nu}\right)^{2}\left(1+\frac{2 \nu}{\theta}\right)^{\theta / \nu} M^{\theta / \nu} P$.

Remark 3.13. When

$$
Q_{r} f(x)=\pi(Q(x, r))^{-1} \sum_{y \in Q(x, r)} f(y) \pi(y)
$$

as in the definition of the $\mathcal{Q}$-Poincare inequality, the first assumption,

$$
\left\|Q_{s} f\right\|_{\infty} \leq M(1+s)^{-\nu}\|f\|_{1},
$$

amounts to a lower bound on the volume of the set $Q(x, r)$. In that case, the second assumption is just the requirement that the $\mathcal{Q}$-Poincaré inequality is satisfied. For an application of this abstract result, see Lemma 6.2.

For the next statement, we assume that $\mu$ is subordinated to $\pi$, i.e., for all $x$, $\sum_{y \in \mathfrak{X}} \mu_{x y} \leq \pi(x)$. We consider the Markov kernel $K$ defined at (3.1) for which $\pi$ is a reversible measure and whose associated Dirichlet form on $L^{2}(\pi)$ is $\mathcal{E}_{\mu}(f, f)=$ $\langle(I-K) f, f\rangle_{\pi}$.

Proposition 3.14 (Diaconis and Saloff-Coste, 1996, Corollary 3.1). Assume that $\mu$ is subordinated to $\pi$ and that

$$
\forall f \in L^{2}(\pi),\|f\|_{2}^{2(1+\theta / \nu)} \leq C\left[\mathcal{E}_{\mu}(f, f)+\frac{1}{N}\|f\|_{2}^{2}\right]\|f\|_{1}^{2 \theta / \nu} .
$$

Then, for all $0 \leq n \leq 2 N$,

$$
\sup _{x, y}\left\{K^{2 n}(x, y) / \pi(y)\right\}=\sup _{x}\left\{K^{2 n}(x, x) / \pi(x)\right\} \leq 2\left(\frac{8 C(1+\nu / \theta)}{n+1}\right)^{\nu / \theta} .
$$

This proposition demonstrates how the Nash inequality provides some control on the decay of the iterated kernel of the Markov chain driven by $K$ over time.

\section{Poincaré and $\mathcal{Q}$-Poincaré inequalities for John domains}

This is a key section of this article as well as one of the most technical. Assuming that $(\mathfrak{X}, \mathfrak{E}, \pi, \mu)$ is adapted, elliptic, and satisfies the doubling property and the ball Poincaré inequality with parameter $\theta$, we derive both a Poincaré inequality (Theorem 4.6) and a $\mathcal{Q}$-Poincaré inequality (Theorem 4.10) on finite John domains. The statement of the Poincaré inequality can be described informally as follows: for a finite domain $U$ in $J(\alpha)$ we have, for all functions $f$ defined on $U$,

$$
\sum_{x \in U}\left|f(x)-f_{U}\right|^{2} \pi(x) \leq C R^{\theta} \mathcal{E}_{\mu, U}(f, f)
$$

where $R$ is the John radius for $U$ and $C$ depends only on $\alpha$ and the constants, coming from doubling, the Poincaré inequality on balls, and ellipticity, which describe the basic properties of $(\mathfrak{X}, \mathfrak{E}, \pi, \mu)$. (Instead of $R$, one can use the intrinsic diameter of $U$ because they are comparable up to a multiplicative constant depending only 
on $\alpha$, see Remark 2.7.) We give an explicit description of the constant $C$ without trying to optimize what can be obtained through the general argument. For many explicit examples running a similar argument while taking advantage of the feature of the example will lead to (much) improved estimates for $C$ in terms of the basic parameters.

These results will be amplified in Section 5 by showing that the same technique works as well for a large class of weights which can be viewed as modifications of the pair $(\pi, \mu)$.

Throughout this section, we fix a finite domain $U$ in $\mathfrak{X}$ with (exterior) boundary $\partial U$ such that $U \in J(o, \alpha, R)$ for some $o \in U$. We also fix a witness family of John-paths $\gamma_{x}$ for each $x \in U$, joining $x$ to $o$ and fulfilling the $\alpha$-John domain condition.

4.1. Poincaré inequality for John domains. Fix a Whitney covering of $U \in J(o, \alpha, R)$,

$$
\mathcal{W}=\left\{B_{i}=B_{x_{i}}^{\eta}=B\left(x_{i}, r_{i}\right): 1 \leq i \leq Q\right\},
$$

with $r_{i}=\eta \delta\left(x_{i}\right) / 4$ and parameter $\eta<1 / 4$. By Lemma 2.14, the collection of balls $B_{i}^{\prime}=3 B_{i}=B\left(x_{i}, 3 r_{i}\right)$ covers $U$, and it is useful to set

$$
\mathcal{W}^{\prime}=\left\{3 B_{i}: 1 \leq i \leq Q\right\} .
$$

Please note that we always think of the elements of $\mathcal{W}, \mathcal{W}^{\prime}$ as balls, each with a specified center and radius, not just subsets.

Lemma 4.1. Any ball $E$ in $\mathcal{W}$ (i.e., $E=B_{i}$ for some $i$ ) has radius $r$ bounded above by $\eta(2 R+1) / 4$.

Proof: By hypothesis, $U \in J(o, \alpha, R)$. Let $R_{o}=\delta(o)$. Any other point $x \in U$ is at distance at most $R$ from $o$. It follows that $\delta(x) \leq R+R_{o} \leq 2 R+1$.

Fix a ball $E_{o}$ in $\mathcal{W}$ such that $3 E_{o}$ contains the point $o$. For any $E=B(z, r) \in \mathcal{W}$, let $\gamma^{E}=\gamma_{z}$ be the John-path from $z$ to $o$ and select a finite sequence

$$
\mathcal{W}^{\prime}(E)=\left(F_{0}^{E}, \ldots, F_{q(E)}^{E}\right)=\left(F_{0}, \ldots, F_{q(E)}\right)
$$

of distinct balls $F_{i}^{E}=F_{i} \in \mathcal{W}^{\prime}$, for $0 \leq i \leq q(E)$ such that $F_{0}^{E}=3 E, F_{q(E)}^{E}=3 E_{o}$, $F_{i}^{E}$ intersects $\gamma^{E}$ and $d\left(F_{i+1}^{E}, F_{i}^{E}\right) \leq 1,0 \leq i \leq q(E)-1$. This is possible since the balls in $\mathcal{W}^{\prime}$ cover $U$. When the ball $E$ is fixed, we drop the superscript $E$ from the notation $F_{i}^{E}$. For each $E \in \mathcal{W}$, the sequence of balls $3 F_{i}^{E}$ (for $1 \leq i \leq q(E)$ ) provides a chain of adjacent balls joining $z$ to $o$ along the John-path $\gamma^{E}$. The union of the balls $6 F_{i}^{E}$ form a carrot-shaped region joining $z$ to $o$ (thin at $z$ and wide at $o$ ). These families of balls are a key ingredient in the following arguments. See Figure 4.11 for an example.

Lemma 4.2. Fix $\eta<1 / 4$ and $\rho \leq 2 / \eta$. The doubling property implies that any point $z \in U$ is contained in at most $D^{1+\log _{2}(4 \rho+3)}$ distinct balls of the form $\rho E$ with $E \in \mathcal{W}$, where $D$ is the volume doubling constant.

Remark 4.3. Note that this property does not necessarily hold if $\rho$ is much larger than $2 / \eta$. This lemma implies that

$$
\sum_{E \in \mathcal{W}} \chi_{\rho E} \leq D^{1+\log _{2}(4 \rho+3)} .
$$




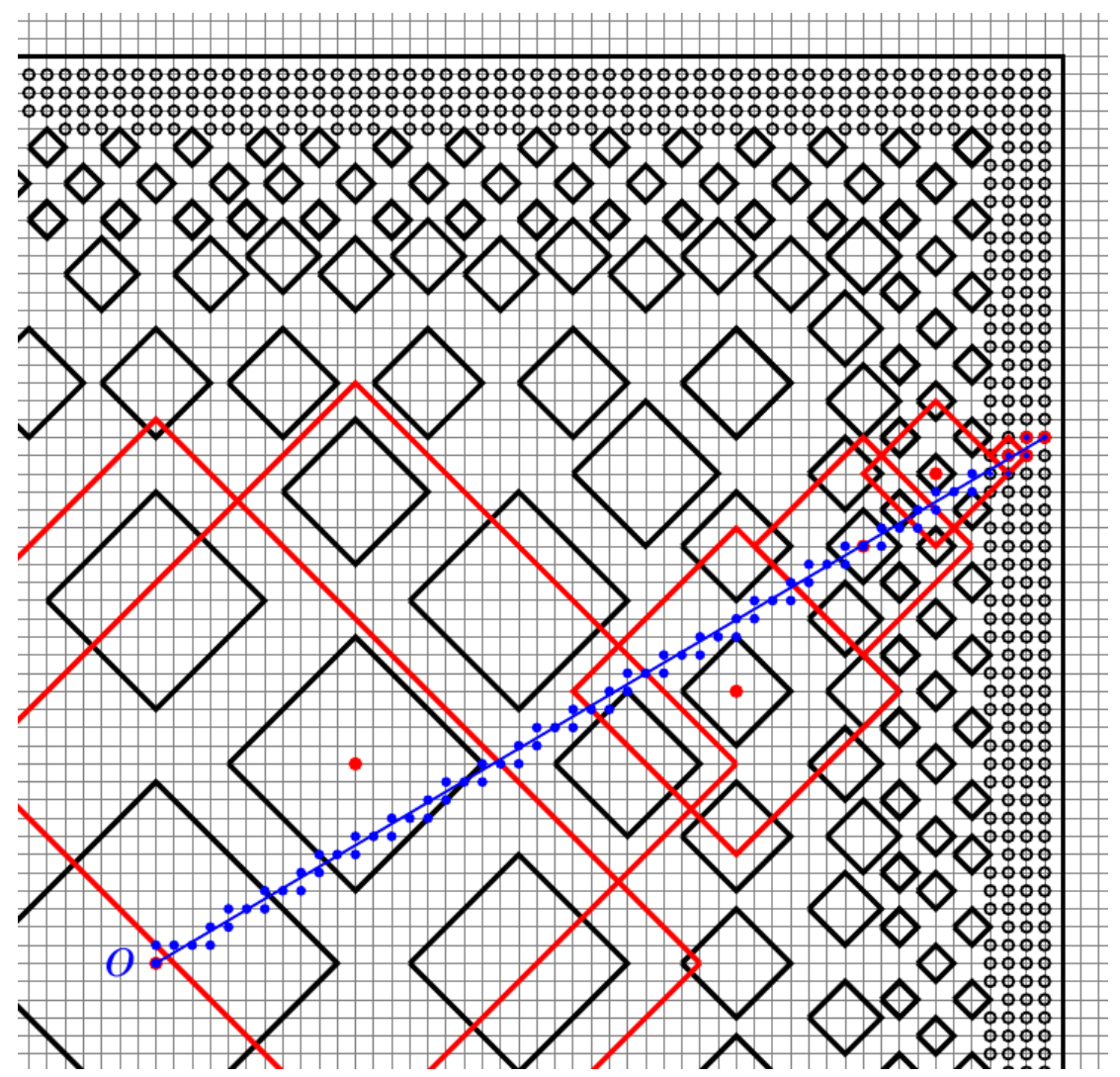

Figure 4.11. A chain of 9 balls $\mathcal{W}^{\prime}(E)=\left\{3 F_{0}, 3 F_{1}, \ldots, 3 F_{q(E)}\right\}$, $q(E)=8$, covering the path $\gamma_{z}$ from $o$ to $z$ (blue points staying close to the straight line from $o$ to $z$ ) with $E=B(z, r) \in \mathcal{W}$, where $W$ is a Whitney covering of the corner of a square. The ball centers are in red. The Whitney parameter $\eta=4 / 5$. The initial Whitney ball $E$ has radius $1 / 5$ so $3 F_{8}=E=\{z\}$. The ball $3 F_{7}, 3 F_{6}$ are also singleton but $3 F_{5}$ has radius $9 / 5$. The ball $3 F_{0}$ is centered at $o$ and has radius 30 .

Proof: Suppose $z \in U$ is contained in $N$ balls $\rho E$ with $E \in \mathcal{W}$, and call them $E_{i}=B\left(x_{i}, r_{i}\right), 1 \leq i \leq N$. By Lemma $2.14(3)$, the radii $r_{i}$ satisfy $r_{i} / r_{j} \leq 3$ (this uses the inequality $\rho \leq 2 / \eta)$ and it follows that

$$
\bigcup_{1}^{N} B\left(x_{i}, r_{i}\right) \subset B\left(x_{j},(4 \rho+3) r_{j}\right) .
$$

Because the balls $E_{i}$ are disjoint, applying this inclusion with $j$ chosen so that $\pi\left(E_{j}\right)=\min \left\{\pi\left(E_{i}\right): 1 \leq i \leq N\right\}$ yields

$$
N \pi\left(E_{j}\right) \leq \pi\left((4 \rho+3) E_{j}\right) \leq D^{1+\log _{2}(4 \rho+3)} \pi\left(E_{j}\right),
$$

which, dividing by $\pi\left(E_{j}\right)$ proves the lemma. 
Lemma 4.4. Fix $\eta<1 / 4$ and $\rho \leq 2 / \eta$. For any ball $E=B(x, r(x)) \in \mathcal{W}$ and any ball $F=B(y, 3 r(y)) \in \mathcal{W}^{\prime}(E)$, where $\mathcal{W}^{\prime}(E)$ is defined in (4.1), we have $E \subset \kappa F$ with $\kappa=3 \alpha^{-1} \eta^{-1}$.

Proof: By construction, there is a point $z$ in $F$ on the John-path $\gamma^{E}$ from $x$ to $o$ and $\delta(z) \geq \alpha(1+d(z, x))$. This implies

$$
4 r(y) / \eta=\delta(y) \geq \delta(z)-3 r(y) \geq \alpha(1+d(z, x))-3 r(y),
$$

that is, $((4 / \eta)+3) r(y) \geq \alpha(1+d(x, z))$. It follows that

$$
x \in B\left(y,\left(3+\alpha^{-1} \eta^{-1}(4+3 \eta)\right) r(y)\right) .
$$

Observe that

$$
\delta(x) \leq \delta(y)+d(x, y) \leq 4 \eta^{-1} r(y)+\left(3+\alpha^{-1} \eta^{-1}(4+3 \eta)\right) r(y)
$$

which gives

$$
r(x)=\eta \delta(x) / 4 \leq r(y)\left(1+\alpha^{-1}(3 \alpha \eta+4+3 \eta) / 4\right) .
$$

Then,

$$
B(x, r(x)) \subset B(y, d(x, y)+r(x))
$$

which gives

$$
B(x, r(x)) \subset B\left(y,\left(4+\alpha^{-1} \eta^{-1}\left(4+3 \eta+\left(3 \alpha \eta^{2}+4 \eta+3 \eta^{2}\right) / 4\right)\right) r(y)\right) .
$$

Because $\alpha \leq 1$ and we assumed $\eta<1 / 4$, we have

$$
4+\alpha^{-1} \eta^{-1}\left(4+3 \eta+\left(3 \alpha \eta^{2}+4 \eta+3 \eta^{2}\right) / 4\right) \leq 4+6 \alpha^{-1} \eta^{-1} \leq 7 \alpha^{-1} \eta^{-1},
$$

and hence $B(x, r(x)) \subseteq \frac{7}{3} \alpha^{-1} \eta^{-1} B(y, r(y))$. For simplicity, we record this as $B(x, r(x)) \subseteq \kappa B(y, r(y))$ with $\kappa=3 \alpha^{-1} \eta^{-1}$.

Lemma 4.5. Fix $\eta \leq 1 / 4$. For each $E \in \mathcal{W}$, the sequence

$$
\mathcal{W}^{\prime}(E)=\left(F_{0}^{E}, \ldots, F_{q(E)}^{E}\right)
$$

has the following properties. Recall that for each $i \in\{0, \ldots, q(E)\}, F_{i}^{E}=B\left(z_{i}^{E}, \rho_{i}^{E}\right)$ with $\rho_{i}^{E}=3 r_{i}^{E}=(3 \eta / 4) \delta\left(z_{i}^{E}\right)$ and that $F_{0}^{E}=3 E, F_{q(E)}^{E}=3 E_{o}$. (We drop the reference to $E$ when $E$ is clearly fixed.)

(1) For each $E$, when $\rho_{i}<1$ we have $B\left(z_{i}, \rho_{i}\right)=\left\{z_{i}\right\}$ and

$$
1+d\left(z_{0}, z_{i}\right) \leq 4 /(3 \alpha \eta) .
$$

(2) For each $E$ and $i \in\{1, \ldots, q(E)-1\}$ such that $\max \left\{\rho_{i}, \rho_{i+1}\right\}<1$, we have

$$
\left|f_{F_{i}}-f_{F_{i+1}}\right|^{2}=\left|f\left(z_{i}\right)-f\left(z_{i+1}\right)\right|^{2} \leq \frac{P_{e}}{\pi\left(z_{i}\right)} \sum_{z \sim z_{i}, z \in U}\left|f(z)-f\left(z_{i}\right)\right|^{2} \mu_{z z_{i}} .
$$

(3) For each $E$ and $i \in\{1, \ldots, q(E)-1\}$ such that $\max \left\{\rho_{i}, \rho_{i+1}\right\} \geq 1$, we have

$$
\left|f_{F_{i}}-f_{F_{i+1}}\right|^{2} \leq 2 D^{6} P\left(8 \rho_{i}\right)^{\theta} \frac{1}{\pi\left(F_{i}\right)} \sum_{x, y \in 8 F_{i}, x \sim y}|f(x)-f(y)|^{2} \mu_{x y},
$$

for any function $f$ on $U$. 
Proof: In the first statement we have $\rho_{i}=\rho\left(z_{i}\right)=3 \eta \delta\left(z_{i}\right) / 4<1$. Because $U \in$ $J(\alpha), E_{0}=B\left(z_{0}, r_{0}\right)=E$ and $z_{i}$ must be on $\gamma^{E}=\gamma_{z_{0}}$,

$$
\delta\left(z_{i}\right) \geq \alpha\left(1+d\left(z_{i}, z_{0}\right)\right) .
$$

It follows that $1+d\left(z_{0}, z_{i}\right) \leq 4 /(3 \alpha \eta)$.

The second statement is clear.

For the third statement, we need some preparation. First we obtain the lower bound

$$
\min \left\{\delta\left(z_{i}\right), \delta\left(z_{i+1}\right)\right\} \geq \frac{5}{6 \eta},
$$

based on the assumption that $\max \left\{\rho_{i}, \rho_{i+1}\right\} \geq 1$. If both $\rho_{i}, \rho_{i+1}$ are at least 1 , there is nothing to prove. If one of them is less than 1 , say $\rho_{i}<1$, then $F_{i}=B\left(z_{i}, \rho_{i}\right)=\left\{z_{i}\right\}$ and $d\left(z_{i}, F_{i+1}\right) \leq 1$. It follows that

$$
\frac{4}{3 \eta} \leq \frac{4}{3 \eta} \rho_{i+1}=\delta\left(z_{i+1}\right) \leq 1+\rho_{i+1}+\delta\left(z_{i}\right) .
$$

But $\rho_{i+1}=(3 \eta / 4) \delta\left(z_{i+1}\right)$, so

$$
\left(1-\frac{3 \eta}{4}\right) \delta\left(z_{i+1}\right) \leq 1+\delta\left(z_{i}\right)
$$

and (using the fact that $\eta \leq 1 / 4$ )

$$
\frac{5}{6 \eta} \leq \frac{4}{3 \eta}-2 \leq \delta\left(z_{i}\right)
$$

This shows that $\min \left\{\rho_{i}, \rho_{i+1}\right\} \geq \frac{5}{8}$ because

$$
\min \left\{\rho_{i}, \rho_{i+1}\right\}=\frac{3 \eta}{4} \min \left\{\delta\left(z_{i}\right), \delta\left(z_{i+1}\right)\right\} \geq \frac{3 \eta}{4} \frac{5}{6 \eta}=5 / 8 .
$$

Next, we show that

$$
F_{i} \cup F_{i+1} \subset 8 F_{i} \cap 8 F_{i+1} \subset U .
$$

By assumption, the balls $B\left(z_{j+1}, 6 r_{j+1}\right)$ and $B\left(z_{j}, 6 r_{j}\right)$ intersect. Applying Lemma 2.14(3) with $\rho=6$ and $\eta \leq 1 / 4$ gives that $5 / 11 \leq r_{j+1} / r_{j} \leq 11 / 5$ and it follows that

$$
\max \left\{\frac{\rho_{i+1}}{\rho_{i}}, \frac{\rho_{i}}{\rho_{i+1}}\right\} \leq 11 / 5
$$

Moreover, because $d\left(F_{i}, F_{i+1}\right) \leq 1$, we have

$$
\max \left\{d\left(z_{i}, z\right): z \in F_{i+1}\right) \leq \rho_{i}+2 \rho_{i+1}+1 \leq 8 \rho_{i}
$$

and similarly,

$$
\max \left\{d\left(z_{i+1}, z\right): z \in F_{i}\right\} \leq \rho_{i+1}+2 \rho_{i}+1 \leq 8 \rho_{i+1} .
$$


It follows that $F_{i} \cup F_{i+1} \subset 8 F_{i} \cap 8 F_{i+1} \subset U$. Now, we are ready to prove the inequality stated in the lemma. Write

$$
\begin{aligned}
\left|f_{F_{i}}-f_{F_{i+1}}\right|^{2} & =\left|\frac{1}{\pi\left(F_{i}\right) \pi\left(F_{i+1}\right)} \sum_{\xi \in F_{i}, \zeta \in F_{i+1}}[f(\xi)-f(\zeta)] \pi(\xi) \pi(\zeta)\right|^{2} \\
& \leq \frac{1}{\pi\left(F_{i}\right) \pi\left(F_{i+1}\right)} \sum_{\xi, \zeta \in 8 F_{i}}|f(\xi)-f(\zeta)|^{2} \pi(\xi) \pi(\zeta) \\
& \leq \frac{2 \pi\left(8 F_{i}\right)}{\pi\left(F_{i}\right) \pi\left(F_{i+1}\right)} \sum_{\xi \in 8 F_{i}}\left|f(\xi)-f_{8 F_{i}}\right|^{2} \pi(\xi) \\
& \leq \frac{2 P \pi\left(8 F_{i}\right)\left(8 \rho_{i}\right)^{\theta}}{\pi\left(F_{i}\right) \pi\left(F_{i+1}\right)} \sum_{x, y \in 8 F_{i}}|f(x)-f(y)|^{2} \mu_{x y} \\
& \leq \frac{2 D^{6} P\left(8 \rho_{i}\right)^{\theta}}{\pi\left(F_{i}\right)} \sum_{x, y \in 8 F_{i}}|f(x)-f(y)|^{2} \mu_{x y}
\end{aligned}
$$

Theorem 4.6. Fix $\alpha, \theta, D, P,>0$. Assume that $(\mathfrak{X}, \mathfrak{E}, \pi, \mu)$ is adapted, elliptic, and satisfies the doubling property with constant $D$ and the ball Poincaré inequality with parameter $\theta$ and constant $P$. Assume that the finite domain $U$ and the point $o \in U$ are such that $U \in J(o, \alpha, R), R>0$. Then there exist a constant $C$ depending only on $\alpha, \theta, D, P$ and such that

$$
\sum_{x \in U}\left|f(x)-f_{U}\right|^{2} \pi(x) \leq P(U) \mathcal{E}_{\mu, U}(f, f)
$$

with

$$
P(U) \leq C R^{\theta} \text { with } C=4^{-\theta} 2 P D^{5}+16 D^{14+2 \log (3 \kappa)} \max \left\{R^{-\theta} P_{e}, 2^{\theta} 2 P D^{6}\right\}
$$

where $\kappa=36 / \alpha$. In particular,

$$
C \leq 17 D^{30+2 \log _{2}(1 / \alpha)} \max \left\{R^{-\theta} P_{e}, 2^{\theta} P\right\} .
$$

Proof: We pick a Whitney covering with $\eta=1 / 12$. Recall from Lemma 4.1 that all balls in $\mathcal{W}$ have radius at most $R / 16$. It suffices to bound $\sum_{x \in U}\left|f(x)-f_{3 E_{o}}\right|^{2} \pi(x)$ because

$$
\sum_{x \in U}\left|f(x)-f_{U}\right|^{2} \pi(x)=\min _{c}\left\{\sum_{x \in U}|f(x)-c|^{2} \pi(x)\right\} .
$$

The balls in $\mathcal{W}^{\prime}$ cover $U$ hence

$$
\sum_{x \in U}\left|f(x)-f_{3 E_{o}}\right|^{2} \pi(x) \leq \sum_{E \in W} \sum_{x \in 3 E}\left|f(x)-f_{3 E_{o}}\right|^{2} \pi(x) .
$$

Next, using the fact that $(a+b)^{2} \leq 2\left(a^{2}+b^{2}\right)$, write

$$
\sum_{E \in W} \sum_{3 E}\left|f-f_{3 E_{o}}\right|^{2} \pi \leq 2\left(\sum_{E \in W} \sum_{3 E}\left|f-f_{3 E}\right|^{2} \pi\right)+2 \sum_{E \in W} \pi(3 E)\left|f_{3 E}-f_{3 E_{o}}\right|^{2} .
$$

We can bound and collect the first part of the right-hand side very easily because, using the Poincaré inequality in balls of radius at most $3 R / 16 \leq R / 4$ and then 
Lemma 4.2, we have

$$
\begin{aligned}
\sum_{E \in W} \sum_{x \in 3 E}\left|f(x)-f_{3 E}\right|^{2} \pi(x) & \leq P(R / 4)^{\theta} \sum_{E \in W} \sum_{\substack{x, y \in 3 E \\
x \sim y}}|f(x)-f(y)|^{2} \mu_{x y} \\
& \leq P D^{5}(R / 4)^{\theta} \mathcal{E}_{\mu, U}(f, f) .
\end{aligned}
$$

This reduces the proof to bounding

$$
\sum_{E \in W} \pi(3 E)\left|f_{3 E}-f_{3 E_{o}}\right|^{2} .
$$

For this, we will use the chain of balls $\mathcal{W}^{\prime}(E)=\left(F_{0}^{E}, \ldots, F_{q(E)}^{E}\right)$ to write

$$
\left|f_{3 E}-f_{3 E_{o}}\right| \leq \sum_{i=0}^{q(E)-1}\left|f_{F_{i}^{E}}-f_{F_{i+1}^{E}}\right| .
$$

Notation. For any function $f$ on $U$ and any ball $F=B(x, \rho) \in \mathcal{W}^{\prime}$ set

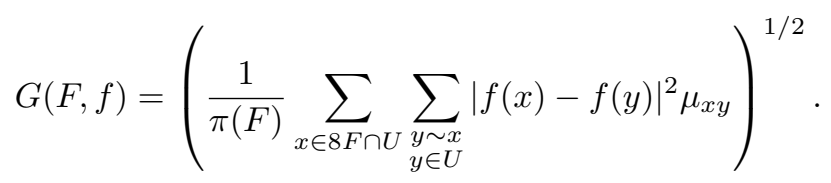

With this notation, Lemma 4.5(2)-(3) yields

$$
\left|f_{F_{i}^{E}}-f_{F_{i+1}^{E}}\right| \leq Q R^{\theta / 2} G\left(F_{i}^{E}, f\right)
$$

where $Q^{2}=\max \left\{R^{-\theta} P_{e}, 2^{\theta} 2 P D^{6}\right\}$. With $\kappa$ as in Lemma 4.4, this becomes

$$
\left|f_{3 E}-f_{3 E_{o}}\right| \mathbf{1}_{E} \leq Q R^{\theta / 2} \sum_{i=0}^{q(E)-1} G\left(F_{i}^{E}, f\right) \mathbf{1}_{E} \mathbf{1}_{\kappa F_{i}^{E}} .
$$

Write

$$
\begin{aligned}
& \sum_{E \in W} \pi(3 E)\left|f_{3 E}-f_{3 E_{o}}\right|^{2} \leq D^{2} \sum_{E \in W} \sum_{x \in U}\left|f_{3 E}-f_{3 E_{o}}\right|^{2} \mathbf{1}_{E}(x) \pi(x) \\
& \leq Q^{2} D^{2} R^{\theta} \sum_{E \in W} \sum_{x \in U}\left|\sum_{i=0}^{q(E)-1} G\left(F_{i}^{E}, f\right) \mathbf{1}_{\kappa F_{i}^{E}}(x)\right|^{2} \mathbf{1}_{E}(x) \pi(x) \\
& \leq Q^{2} D^{2} R^{\theta} \sum_{E \in W} \sum_{x \in U}\left|\sum_{F \in \mathcal{W}^{\prime}} G(F, f) \mathbf{1}_{\kappa F}(x)\right|^{2} \mathbf{1}_{E}(x) \pi(x) \\
& \leq Q^{2} D^{2} R^{\theta} \sum_{x \in \mathfrak{X}}\left|\sum_{F \in \mathcal{W}^{\prime}} G(F, f) \mathbf{1}_{\kappa F}(x)\right|^{2} \pi(x)
\end{aligned}
$$

where the last step follows from the observation that $\sum_{E \in \mathcal{W}} \mathbf{1}_{E} \leq 1$ because the balls in $\mathcal{W}$ are pairwise disjoint. 
By Proposition B.1 and the fact that the balls in $\mathcal{W}$ are disjoint, we have

$$
\begin{aligned}
& \sum_{x \in \mathfrak{X}}\left|\sum_{E \in \mathcal{W}} G(3 E, f) \mathbf{1}_{3 \kappa E}(x)\right|^{2} \pi(x) \\
& \leq 8 D^{4+2 \log _{2}(3 \kappa)} \sum_{\mathfrak{X}}\left|\sum_{E \in \mathcal{W}} G(3 E, f) \mathbf{1}_{E}(x)\right|^{2} \pi(x) \\
& =8 D^{4+2 \log _{2}(3 \kappa)} \sum_{E \in \mathcal{W}} G(3 E, f)^{2} \pi(E) \\
& \quad=8 D^{4+2 \log _{2}(3 \kappa)} \sum_{E \in \mathcal{W} x, y \in 24 E \cap U}|f(x)-f(y)|^{2} \mu_{x y}
\end{aligned}
$$

By Lemma 4.2 (note that $2 / \eta=24$ ), for each $x \in \mathfrak{X}$, there are at most $D^{8}$ balls $E$ in $\mathcal{W}$ such that $24 E$ contains $x$. This yields

$$
\sum_{x \in \mathfrak{X}}\left|\sum_{F \in \mathcal{W}} G(2 F, f) \mathbf{1}_{3 \kappa F}(x)\right|^{2} \pi(x) \leq 8 D^{12+2 \log (3 \kappa)} \mathcal{E}_{U, \mu}(f, f) .
$$

Collecting all terms gives Theorem 4.6 as desired.

4.2. $\mathcal{Q}$-Poincaré inequality for John domains. For any $s \geq 1$, fix a scale-s Whitney covering $\mathcal{W}_{s}$ with Whitney parameter $\eta<1 / 4$. For our purpose, we can restrict ourselves to integer parameters $s$ no greater than $2 R+1$ which results in making only finitely many choice of coverings. Recall that $\mathcal{W}_{s}$ is the disjoint union of $\mathcal{W}_{=s}$ (balls of radius exactly $s$ ) and $\mathcal{W}_{<s}$ (balls of radius strictly less than $s$ ). As before, we denote by $\mathcal{W}_{s}^{\prime}, \mathcal{W}_{=s}^{\prime}$ and $\mathcal{W}_{<s}^{\prime}$, the sets of balls obtained by tripling the radius of the balls in $\mathcal{W}_{s}, \mathcal{W}_{=s}$ and $\mathcal{W}_{<s}$.

Fix a ball $E_{o}^{s}$ in $\mathcal{W}_{s}$ such that $3 E_{o}^{s}$ contains the point $o$. For any $E=B(z, r)$ $\in \mathcal{W}_{s}$, select a finite sequence

$$
\mathcal{W}_{s}^{\prime}(E)=\left(F_{0}^{s, E}, \ldots, F_{q_{s}(E)}^{s, E}\right)=\left(F_{0}, \ldots, F_{q(E)}\right)
$$

of distinct balls $F_{i}^{s, E}=F_{i} \in \mathcal{W}_{s}^{\prime}\left(\right.$ for $\left.0 \leq i \leq q_{s}(E)\right)$ such that $F_{0}^{s, E}=3 E$, $F_{q(E)}^{E}=3 E_{o}^{s}, F_{i}^{s, E}$ intersects $\gamma^{E}$ and $d\left(F_{i+1}^{s, E}, F_{i}^{s, E}\right) \leq 1\left(0 \leq i \leq q_{s}(E)-1\right)$. This is obviously possible since the balls in $\mathcal{W}_{s}^{\prime}$ cover $U$. When the parameter $s$ and the ball $E$ are fixed, we drop the supscripts $s, E$ from the notation $F_{i}^{s, E}$. We only need a portion of this sequence, namely,

$$
W_{<s}^{\prime}(E)=\left(F_{0}^{s, E}, \ldots, F_{q_{s}^{*}(E)}^{s, E}\right)
$$

where $q_{s}^{*}(E)$ is the smallest index $j$ such that $r_{j}=s$. If no such $j$ exists, set $q_{s}^{*}(E)=q(E)$. For future reference, we call these sequences of balls local s-chains. Namely, the sequence $W_{<s}^{\prime}(E)$ is the local s-chain for $E$ at scale $s$.

We set

$$
F(s, E)=F_{q_{s}^{*}(E)}^{s, E},
$$

to be the last ball in the local s-chain of $E$. For each $x$, choose a ball $E(s, x) \in \mathcal{W}_{s}$ with maximal radius among those $E \in \mathcal{W}_{s}$ such that $3 E$ contains $x$ and set

$$
F(s, x)=\left\{\begin{array}{cl}
3 E(s, x) & \text { when } x \in \bigcup_{E \in \mathcal{W}_{=s}} 3 E \\
F(s, E(s, x)) & \text { otherwise. }
\end{array}\right.
$$


The ball $F(s, x)$ is, roughly speaking, chosen among those balls of radius $3 s$ in the Whitney covering that are not too far from $x$ and away from the boundary of $U$ for points $x$ near the boundary, where the Whitney balls have radius less than $s$, $F(s, x)$ is the last ball in the local s-chain of $E \in \mathcal{W}_{s}$, where $3 E$ covers $x$.

Definition 4.7. For $s \in[0,1]$, set $Q_{s}=I$ (i.e., $Q_{s} f=f$ ). For any $s>1$, define the averaging operator

$$
Q_{s} f(x)=\sum_{y \in u} Q_{s}(x, y) f(y) \pi(y)
$$

by setting

$$
Q_{s}(x, y)=\frac{1}{\pi(F(s, x))} \mathbf{1}_{F(s, x)}(y)
$$

Next we collect the $s$-version of the statements analogous to Lemmas 4.2 and 4.4. The proofs are the same.

Lemma 4.8. Fix $\eta<1 / 4$ and $\rho \leq 2 / \eta$. For any $s>0$, the following properties hold.

(1) Any point $z \in U$ is contained in at most $D^{1+\log _{2}(4 \rho+3)}$ distinct balls $\rho E$ with $E \in \mathcal{W}_{s}$.

(2) For any ball $E=B(x, r(x)) \in \mathcal{W}_{<s}$ and any ball $F=B(y, 3 r(y)) \in \mathcal{W}_{<s}^{\prime}(E)$ we have $E \subset \kappa F$ with $\kappa=3 \alpha^{-1} \eta^{-1}$.

The $s$-version of Lemma 4.5 is as follows. The proof is the same.

Lemma 4.9. Fix $\eta \leq 1 / 4$. For each $s \geq 1$, and $E \in \mathcal{W}_{<s}$, the sequence

$$
\mathcal{W}_{<s}^{\prime}(E)=\left(F_{0}^{s, E}, \ldots, F_{q_{s}^{*}(E)}^{s, E}\right)
$$

has the following properties. Set $i \in\left\{0, \ldots, q_{s}^{*}(E)\right\}, F_{i}^{s, E}=B\left(z_{i}^{s, E}, \rho_{i}^{s, E}\right)$ with $\rho_{i}^{s, E}=3 r_{i}^{s, E}=3 \min \left\{s, \eta \delta\left(z_{i}^{E}\right) / 4\right\}$ and that $F_{0}^{s, E}=3 E, F_{q_{s}^{*}(E)}^{s, E}=F(s, E)$. We drop the reference to $s$ and $E$ when they are clearly fixed.

(1) For each $E \in W_{<s}$, when $\rho_{i}<1$ we have $B\left(z_{i}, \rho_{i}\right)=\left\{z_{i}\right\}$ and

$$
1+d\left(z_{0}, z_{i}\right) \leq 4 /(3 \alpha \eta) \text {. }
$$

(2) For each $E \in W_{<s}$ and $i \in\left\{1, \ldots, q_{s}^{*}(E)-1\right\}$ such that $\max \left\{\rho_{i}, \rho_{i+1}\right\}<1$, we have

$$
\left|f_{F_{i}}-f_{F_{i+1}}\right|^{2}=\left|f\left(z_{i}\right)-f\left(z_{i+1}\right)\right|^{2} \leq \frac{P_{e}}{\pi\left(z_{i}\right)} \sum_{\substack{z \sim z_{i} \\ z \in U}}\left|f(z)-f\left(z_{i}\right)\right|^{2} \mu_{z z_{i}} .
$$

(3) For each $E \in W_{<s}$ and $i \in\left\{1, \ldots, q_{s}^{*}(E)-1\right\}$ such that $\max \left\{\rho_{i}, \rho_{i+1}\right\} \geq 1$ we have $\min \left\{\delta\left(z_{i}\right), \delta\left(z_{i+1}\right)\right\} \geq 4 /(9 \eta), \min \left\{\rho_{i}, \rho_{i+1}\right\} \geq 1 / 3$ and

$$
F_{i} \cup F_{i+1} \subset 8 F_{i} \subset U \text {. }
$$

Furthermore, for any function $f$ on $U$,

$$
\left|f_{F_{i}}-f_{F_{i+1}}\right|^{2} \leq 2 D^{6} P\left(8 \rho_{i}\right)^{\theta} \frac{1}{\pi\left(F_{i}\right)} \sum_{\substack{x \sim y \\ x, y \in 8 F_{i}}}|f(x)-f(y)|^{2} \mu_{x y} .
$$


Theorem 4.10. Fix $\alpha, \theta, D, P_{e}, P>0$. Assume that $(\mathfrak{X}, \mathfrak{E}, \pi, \mu)$ is adapted, elliptic and, satisfies the doubling property with constant $D$ and the ball Poincaré inequality with parameter $\theta$ and constant $P$. Assume that the finite domain $U$ and the point $o \in U$ are such that $U \in J(o, \alpha, R), R>0$. Then there exists a constant $C$ depending only on $\alpha, \theta, D, P$ and such that

$$
\forall s>0, \quad \sum_{x \in U}\left|f(x)-Q_{s} f(x)\right|^{2} \pi(x) \leq C s^{\theta} \mathcal{E}_{\mu, U}(f, f)
$$

with

$$
C=3^{\theta} 7 P D^{5}+16 D^{14+2 \log (3 \kappa)} \max \left\{P_{e}, 8^{\theta} 2 P D^{6}\right\}
$$

where $\kappa=36 / \alpha$.

Proof: The conclusion trivially holds when $s \in[0,1]$ because $Q_{s} f=f$ in this case. For $s>1$, as in the proof of Theorem 4.6, we pick a Whitney covering with $\eta=1 / 12$. We need to bound

$$
\begin{aligned}
\sum_{x \in U}\left|f(x)-Q_{s} f(x)\right|^{2} \pi(x)= & \sum_{E \in \mathcal{W}_{s}} \sum_{\substack{x \in 3 E \\
E=E(s, x)}}\left|f(x)-f_{F(s, E)}\right|^{2} \pi(x) \\
= & \sum_{E \in \mathcal{W}_{=s}} \sum_{\substack{x \in 3 E \\
E=E(s, x)}}\left|f(x)-f_{3 E}\right|^{2} \pi(x) \\
& +\sum_{E \in \mathcal{W}_{<s}} \sum_{\substack{x \in 3 E \\
E=E(s, x)}}\left|f(x)-f_{F(s, E)}\right|^{2} \pi(x) \\
\leq & \sum_{E \in \mathcal{W}_{=s}} \sum_{x \in 3 E}\left|f(x)-f_{3 E}\right|^{2} \pi(x) \\
& +\sum_{E \in \mathcal{W}_{<s}} \sum_{x \in 3 E}\left|f(x)-f_{F(s, E)}\right|^{2} \pi(x) .
\end{aligned}
$$

Note that, in the first two lines, we are only summing over the $x$ such that $E=$ $E(s, x)$ i.e., $E \in \mathcal{W}_{s}$ is the selected ball of radius $s$ which covers $x$. That way, $x \in U$ appears once in the sum. In the third line, we expand the sum and each $x$ may appear multiple times.

We can bound and collect the first part of the right-hand side of the last inequality using the Poincaré inequality on balls of radius $3 s$ and Lemma 4.8(1),

$$
\begin{aligned}
\sum_{E \in W=s} \sum_{x \in 3 E}\left|f(x)-f_{3 E}\right|^{2} \pi(x) & \leq P(3 s)^{\theta} \sum_{E \in W=s} \sum_{\substack{x, y \in 3 E \\
x \sim y}}|f(x)-f(y)|^{2} \mu_{x y} \\
& \leq 3^{\theta} P D^{5} s^{\theta} \mathcal{E}_{\mu, U}(f, f) .
\end{aligned}
$$

This reduces the proof to bounding

$$
\begin{aligned}
& \sum_{E \in W_{<s}} \sum_{x \in 3 E}\left|f(x)-f_{F(s, x)}\right|^{2} \pi(x) \\
& \leq 2 \sum_{E \in \mathcal{W}_{<s}}\left(\sum_{x \in 3 E}\left|f(x)-f_{3 E}\right|^{2} \pi(x)+\pi(3 E)\left|f_{3 E}-f_{F(s, x)}\right|^{2}\right) .
\end{aligned}
$$


The first part of the right-hand side is, again, easily bounded by

$$
\begin{aligned}
2 \sum_{E \in \mathcal{W}_{<s}} \sum_{x \in 3 E}\left|f(x)-f_{3 E}\right|^{2} \pi(x) & \leq 3^{1+\theta} P s^{\theta} \sum_{E \in \mathcal{W}_{<s}} \sum_{x, y \in 3 E, x \sim y}|f(x)-f(y)|^{2} \mu_{x y} \\
& \leq 3^{1+\theta} P D^{5} s^{\theta} \mathcal{E}_{\mu, U}(f, f) .
\end{aligned}
$$

The second part is

$$
2 \sum_{E \in \mathcal{W}_{<s}} \pi(3 E)\left|f_{3 E}-f_{F(s, x)}\right|^{2}
$$

for which we use the chain of balls $\mathcal{W}_{s}^{\prime}(E)=\left(F_{0}^{s, E}, \ldots, F_{q_{s}^{*}(E)}^{s, E}\right)$ to write

$$
\left|f_{3 E}-f_{F(s, E)}\right| \leq \sum_{i=0}^{q_{s}^{*}(E)-1}\left|f_{F_{i}^{s, E}}-f_{F_{i+1}^{s, E}}\right| .
$$

Lemma 4.9(2)-(3) and the notation $G(F, f)$ introduced for the proof of Theorem 4.6 yields

$$
\left|f_{F_{i}^{s, E}}-f_{F_{i+1}^{s, E}}\right| \leq Q s^{\theta / 2} G\left(F_{i}^{s, E}, f\right), \quad Q^{2}=\max \left\{s^{-\theta} P_{e}, 8^{\theta} 2 P D^{6}\right\}
$$

and, with $\kappa$ as in Lemma 4.8(2),

$$
\left|f_{3 E}-f_{F(s, E)}\right| \mathbf{1}_{E} \leq Q s^{\theta / 2} \sum_{i=0}^{q_{s}^{*}(E)-1} G\left(F_{i}^{s, E}, f\right) \mathbf{1}_{E} \mathbf{1}_{\kappa F_{i}^{s, E}} .
$$

Using this estimate, the same argument used at the end of the proof of Theorem 4.6 (and based on Proposition B.1) gives

$$
2 \sum_{E \in \mathcal{W}_{<s}} \pi(3 E)\left|f_{3 E}-f_{F(s, x)}\right|^{2} \leq 16 Q^{2} D^{13+2 \log _{2} 3 \kappa} s^{\theta} \mathcal{E}_{\mu, U}(f, f) .
$$

\section{Adding weights and comparison argument}

Comparison arguments are very useful in the study of ergodic finite Markov chains (see Diaconis and Saloff-Coste, 1993b and Diaconis and Saloff-Coste, 1993a). This section uses these ideas in the present context. The results here are used in Section 6 to study the rates of convergence for Metropolis type chains and in Sections 7 and 8 for studying Markov chains which are killed on the boundary.

By their very nature, the (almost identical) proofs of Theorems 4.6 and 4.10 allow for a number of important variants. In this subsection, we discuss transforming the pair $(\pi, \mu)$ into a pair $(\widetilde{\pi}, \widetilde{\mu})$ so that the proofs of the preceding section yield Poincaré type inequalities (including $Q$-type) for this new pair.

Definition 5.1. Let $U$ be a finite domain in $(\mathfrak{X}, \mathfrak{E}, \pi, \mu)$. Let $(\widetilde{\pi}, \widetilde{\mu})$ be given on $\left(U, \mathfrak{E}_{U}\right)$. We say that the pair $(\widetilde{\pi}, \widetilde{\mu})(\eta, A)$-dominates the pair $(\pi, \mu)$ in $U$ if, for any ball $E=B(z, r) \subset U$ with $r \leq 6 \eta \delta(z)$, we have

$$
\sup \left\{\frac{\widetilde{\pi}(x)}{\pi(x)}: x \in B\right\} \leq A \inf \left\{\frac{\widetilde{\mu}_{x y}}{\mu_{x y}}: x \in B,\{x, y\} \in E\right\},
$$


Remark 5.2. If $\eta \geq 1 / 6$, this property is very strong and not very useful. We will use it with $\eta \leq 1 / 12$ so that each of the balls considered is far from the boundary relative to the size of its radius. The size of balls for which this property is required, namely, balls such that $r \leq 6 \eta \delta(z)$ is dictated by the fact the we will have to use this property for the balls $24 E$ where $E=B(z, r(z))$ is a ball that belong to an $\eta$-Whitney covering of $U$. See Lemma 4.9(3). By construction, such a ball $E$ will satisfy $r(z)=\eta \delta(z) / 4$ and $r=24 r(z)$ satisfies $r=6 \eta \delta(x)$.

The following obvious lemma justifies the above definition.

Lemma 5.3. Assume that $(\tilde{\pi}, \widetilde{\mu})(\eta, A)$-dominates the pair $(\pi, \mu)$ in $U$.

(1) If $(\pi, \mu)$ is $P_{e}$-elliptic then $(\tilde{\pi}, \widetilde{\mu})$ is $A P_{e}$-elliptic on $U$.

(2) If $B=B(z, r)$ is a ball such that $r \leq 6 \eta \delta(z)$ and the Poincaré inequality

$$
\sum_{x \in B}\left|f(x)-f_{B}\right|^{2} \pi \leq P(B) \sum_{x, y \in B}|f(x)-f(y)|^{2} \mu_{x y}
$$

holds on $B$ then

$$
\sum_{x \in B}\left|f-\widetilde{f}_{B}\right|^{2} \widetilde{\pi} \leq \sum_{x \in B}\left|f(x)-f_{B}\right|^{2} \widetilde{\pi} \leq A P(B) \sum_{x, y \in B}|f(x)-f(y)|^{2} \widetilde{\mu}_{x y}
$$

where $\widetilde{f}_{B}$ is the mean of $f$ over $B$ with respect to $\tilde{\pi}$ and $f_{B}$ is the mean of $f$ over $B$ with respect to $\pi$.

Definition 5.4. Assume that $U$ is a connected subset of $(\mathfrak{X}, \mathfrak{E})$ with internal boundary $\delta U=\{x \in U: \exists y \in \mathfrak{X} \backslash U,\{x, y\} \in \mathfrak{E}\}$. For each $x \in \delta U$, introduce an auxiliary vertex $x^{c}$ and set

$$
\mathfrak{U}=U \cup\left\{x^{c}: x \in \delta U\right\}, \mathfrak{E}_{\mathfrak{U}}=\mathfrak{E}_{U} \cup\left\{\left\{x, x^{c}\right\}: x \in \delta U\right\},
$$

so that $\mathfrak{U}$ has an additional copy of $\delta U$ attached to $\delta U$. By inspection, a domain $U$ is in $J(\mathfrak{X}, \mathfrak{E}, \alpha, o, R)$ if and only if $U \in J\left(\mathfrak{U}, \mathfrak{E}_{\mathfrak{U}}, \alpha, o, R\right)$. If $\widetilde{\pi}$ is a measure on $U$ then we can extend this measure to a measure on $\mathfrak{U}$, which we still call $\tilde{\pi}$, by setting $\widetilde{\pi}\left(x^{c}\right)=\widetilde{\pi}(x), x \in \delta U$. If $\widetilde{\pi}$ is $\widetilde{D}$-doubling on $\left(U, \mathfrak{E}_{\mathfrak{U}}\right)$ then its extension is $2 \widetilde{D}$-doubling on $\left(\mathfrak{U}, \mathfrak{E}_{U}\right)$.

5.1. Adding weight under the doubling assumption for the weighted measure.

Theorem 5.5. Referring to the setting of Theorems 4.6-4.10, assume further that we are given $\eta \in(0,1 / 12)$ and a pair $(\tilde{\pi}, \widetilde{\mu})$ on $U$ which dominates $(\pi, \mu)$ with constants $(\eta, A)$ and such that $\widetilde{\pi}$ is $\widetilde{D}$-doubling on $\left(U, \mathfrak{E}_{U}\right)$. Then there exists a constant $C$ depending only on $\eta, A, \alpha, \theta, \widetilde{D}, P, P_{e}$ such that

$$
\forall s>0, \quad \sum_{x \in U}\left|f(x)-\widetilde{Q}_{s} f(x)\right|^{2} \widetilde{\pi}(x) \leq C s^{\theta} \mathcal{E}_{\widetilde{\mu}, U}(f, f) .
$$

We can take

$$
C=7\left(3^{\theta} P A(2 \widetilde{D})^{5}\right)+16 A(2 \widetilde{D})^{14+2 \log (2 \kappa)} \max \left\{P_{e}, 8^{\theta} 2 P(2 \widetilde{D})^{6}\right\}
$$

where $\kappa=7 /(\alpha \eta)$.

Here $\widetilde{Q}_{s}$ is as in Definition 4.7 with $\tilde{\pi}$ instead of $\pi$. In particular,

$$
\sum_{x \in U}\left|f(x)-\widetilde{f}_{U}\right|^{2} \widetilde{\pi}(x) \leq C R^{\theta} \mathcal{E}_{\widetilde{\mu}, U}(f, f) .
$$


Proof: Follow the proofs of Theorems 4.6-4.10, using a $\eta$-Whitney covering with $\eta$ small enough that the Poincaré inequalities on Whitney balls (in fact, on double Whitney balls) holds for the pair $(\tilde{\pi}, \widetilde{\mu})$ by Lemma 5.3. To make the argument go as smoothly as possible, use the construction of $\left(\mathfrak{U}, \mathfrak{E}_{\mathfrak{U}}\right)$ in Definition 5.4. The proof proceeds as before with $(\tilde{\pi}, \widetilde{\mu})$ instead of $(\pi, \mu)$. The full strength of the assumption that $\tilde{\pi}$ is doubling is key in applying Proposition B.1 in this context.

\subsection{Adding weight without the doubling assumption for the weighted measure.}

Definition 5.6. Let $\psi: U \rightarrow(0, \infty)$ be a positive function on $U$ (we call it a weight). We say that $\psi$ is $A$-doubling on $U$ if the measure $\psi \pi$ is doubling on $\left(U, \mathfrak{E}_{U}\right)$ with constant $A$.

Definition 5.7. Let $\psi: U \rightarrow(0, \infty)$ be a positive function on $U$. We say that $\psi$ is $(\eta, A)$-regular on $U$ if

$$
\psi(x) \leq A \psi(y) \text { for all }\{x, y\} \in \mathfrak{E}_{U},
$$

and, for any ball $E=B(z, r) \subset U$ with $r \leq 6 \eta \delta(z)$, we have

$$
\max _{E}\{\psi\} \leq A \min _{E}\{\psi\}
$$

Remark 5.8. Assume that $\psi$ is $(\eta, A)$-regular and consider any pair $(\tilde{\pi}, \tilde{\mu})$ on $\left(U, \mathfrak{E}_{U}\right)$ such that

$$
\tilde{\pi} \leq \psi \pi, \quad \mu_{x y} \psi(x) \leq A^{\prime} \widetilde{\mu}_{x y}
$$

Then the pair $(\tilde{\pi}, \widetilde{\mu})\left(\eta, A A^{\prime}\right)$-dominates $(\pi, \mu)$. For instance we can set $\tilde{\pi}=\psi \pi$ and take $\tilde{\mu}$ to be given by one of the following choices:

$$
\mu_{x y} \sqrt{\psi(x) \psi(y)}, \quad \tilde{\mu}_{x y}=\mu_{x y} \min \{\psi(x), \psi(y)\} \text { or } \widetilde{\mu}_{x y}=\mu_{x y} \max \{\psi(x), \psi(y)\} .
$$

In these three cases $A^{\prime}=\sqrt{A}, A^{\prime}=A$ and $A^{\prime}=1$, respectively.

Definition 5.9. Fix $\eta \in(0,1 / 8)$. Let $\psi$ be a weight on a finite domain $U$ such that $\psi$ is $(\eta, A)$-regular on $U$. Assume $U$ is a John domain, $U \in J(\alpha, o, R)$, equipped with John paths $\gamma_{x}$ joining $x$ to $o, x \in U$, and a family of $\eta$-Whitney coverings $\mathcal{W}_{s}, s \geq 1$. We say that $\psi$ is $\left(\omega, A_{1}\right)$-controlled if, for any local s-chain $\mathcal{W}_{<s}^{\prime}(E)=$ $\left(F_{0}^{s, E}, \ldots, F_{q_{s}^{*}(E)}^{s, E}\right)$ with $F_{i}^{s, E}=B\left(x_{i}, 3 r\left(x_{i}\right)\right), 0 \leq i \leq q_{s}^{*}(E)$, we have

$$
\forall s \geq 1, \quad \forall i \in\left\{0, \ldots, q_{s}^{*}(E)\right\}, \quad \psi\left(x_{0}\right) \leq A_{1} s^{\omega} \psi\left(x_{i}\right) .
$$

When we say that an $(\eta, A)$-regular weight $\psi$ on $U \in J(\alpha, o, R)$ is $\left(\omega, A_{1}\right)$-controlled, we assume implicitly that a family of $\eta$-Whitney coverings $\mathcal{W}_{s}, s \geq 1$ has been chosen.

Remark 5.10. When $\omega=0$, the weight $\psi$ is essentially increasing along the John path joining Whitney balls to $o$.

Theorem 5.11. Given the setting of Theorems 4.6 and 4.10, assume further that we are given $\eta \in(0,1 / 12)$ and a weight $\psi$ on $U$ such that $\psi$ is $(\eta, A)$-regular and $\left(\omega, A_{1}\right)$-controlled. Set $\widetilde{\pi}=\psi \pi$ and let $\widetilde{\mu}$ be a weight defined on $\mathfrak{E}_{U}$ such that

$$
\forall x, y \in U, \quad \psi(x) \mu_{x y} \leq A_{2} \widetilde{\mu}_{x y} .
$$

Then there exist a constant $C$ depending only on $\eta, \alpha, \theta, A, A_{1}, A_{2}, D, P$ and such that

$$
\forall s>0, \quad \sum_{x \in U}\left|f(x)-\widetilde{Q}_{s} f(x)\right|^{2} \widetilde{\pi}(x) \leq C s^{\theta+\omega} \mathcal{E}_{\widetilde{\mu}, U}(f, f) .
$$


Here $\widetilde{Q}_{s}$ is as in Definition 4.7 with $\widetilde{\pi}$ instead of $\pi$. The constant $C$ can be taken to be

$$
C=C=7 A A_{2}\left(3^{\theta} P D^{5}\right)+16 D^{14+2 \log (2 \kappa)} A^{3} A_{1} A_{2} \max \left\{P_{e}, 8^{\theta} 2 P A^{2} D^{6}\right\}
$$

where $\kappa=7 /(\alpha \eta)$. In particular,

$$
\sum_{x \in U}\left|f(x)-\widetilde{f}_{U}\right|^{2} \widetilde{\pi}(x) \leq C R^{\theta+\omega} \mathcal{E}_{\widetilde{\mu}, U}(f, f) .
$$

Proof: (The case $s \in[0,1]$ is trivial and we can assume $s>1$ ). This result is a bit more subtle than the previous result because the measure $\tilde{\pi}$ may not be doubling. However, because $\psi$ is $(\eta, A)$-regular and $\widetilde{\mu}$ satisfies (5.1), it follows from Remark 5.8 that $(\widetilde{\pi}, \widetilde{\mu})\left(\eta, A A_{2}\right)$-dominates $(\pi, \mu)$. By Lemma 5.3 this implies that $(\widetilde{\pi}, \widetilde{\mu})$ is $A A_{2} P_{e}$-elliptic and the $\theta$-Poincare inequality on balls $B(z, r)$ such that $r \leq \eta \delta(z), z \in U$, with constant $P A A_{2}$. Using the notation $\widetilde{f}_{B}$ for the mean of $f$ over $B$ with respect to $\widetilde{\pi}$, we also have, for any ball $E$ in $\mathcal{W}_{<s}$ and its local s-chain $\mathcal{W}_{<s}^{\prime}(E)=\left(F_{i}^{s, E}\right)_{0}^{q_{s}^{*}(E)}$ with $F_{i}^{s, E}=B\left(x_{i}, 3 r\left(x_{i}\right)\right), F_{0}^{s, E}=3 E$,

$$
\left|\widetilde{f}_{F_{i}^{s, E}}-\widetilde{f}_{F_{i+1}^{s, E}}\right| \leq Q s^{\theta / 2} \widetilde{G}\left(F_{i}^{s, E}, f\right)
$$

where $\widetilde{G}$ is defined just as $G$ but with respect to the pair $(\widetilde{\pi}, \widetilde{\mu})$. Here we can take

$$
Q^{2}=A A_{2} \max \left\{P_{e}, 8^{\theta} 2 P A^{2} D^{6}\right\} .
$$

In this computation (see the proof of Lemma 4.5), we have had to estimate $\widetilde{\pi}\left(8 F_{j}\right) / \widetilde{\pi}\left(F_{j}\right)$ by $A D^{3}$ using the doubling property of $\pi$ and the fact that $\psi$ is $(\eta, A)$-regular (in words, what is used here is the fact that, because $\psi$ is $(\eta, A)$ regular, $\widetilde{\pi}$ is doubling on balls that are far away from the boundary even so it is not necessarily globally doubling on $\left.\left(U, \mathfrak{E}_{U}\right)\right)$.

Next, set

$$
G^{*}(F, f)=\left(\frac{1}{\pi(F)} \sum_{x \in 8 F \cap U} \sum_{\substack{y \sim x \\ y \in U}}|f(x)-f(y)| \widetilde{\mu}_{x y}\right)^{1 / 2} .
$$

This differs from $\widetilde{G}(F, f)$ only by the use of $\pi$ instead of $\widetilde{\pi}$ in the fraction appearing in front of the summations (but note that this quantity involves the edge weight $\widetilde{\mu})$. Now, we have

$$
\left|\widetilde{f}_{F_{i}^{s, E}}-\widetilde{f}_{F_{i+1}^{s, E}}\right|\left(\frac{\widetilde{\pi}(3 E)}{\pi(3 E)}\right)^{1 / 2} \leq A \sqrt{A_{1}} Q s^{(\theta+\omega) / 2} G^{*}\left(F_{i}^{s, E}, f\right)
$$

because

$$
\frac{\widetilde{\pi}(3 E)}{\pi(3 E)} \leq A \psi\left(x_{0}\right) \leq A A_{1} s^{\omega} \psi\left(x_{i}\right) \leq A^{2} A_{1} s^{\omega} \frac{\widetilde{\pi}\left(F_{i}^{s, E}\right)}{\pi\left(F_{i}^{s, E}\right)} .
$$

This gives

$$
\begin{aligned}
& \left|\widetilde{f}_{3 E}-\widetilde{f}_{F(s, E)}\right|\left(\frac{\widetilde{\pi}(3 E)}{\pi(3 E)}\right)^{1 / 2} \mathbf{1}_{E} \\
& \quad \leq A^{2} A_{1} Q s^{(\theta+\omega) / 2} \sum_{i=0}^{q_{s}^{*}(E)-1} G^{*}\left(F_{i}^{s, E}, f\right) \mathbf{1}_{E} \mathbf{1}_{\kappa F_{i}^{s, E}} .
\end{aligned}
$$


To finish the proof, we square both sides, multiply by $\pi(3 E)$, and proceed as at the end of the proof of Theorem 4.10, using the doubling property of $\pi$.

5.3. Regular weights are always controlled. The following lemma is a version of a well-known fact concerning chains of Whitney balls in John domains.

Lemma 5.12. Assume that $(\mathfrak{X}, \mathfrak{E}, \pi)$ is doubling with constant D. Fix $\eta \in(0,1 / 8)$. Let $\psi$ be a weight on a finite domain $U$ such that $\psi$ is $(\eta, A)$-regular on $U$ and $U$ is a John domain, $U \in J(\alpha, o, R)$, equipped with John paths $\gamma_{x}$ joining $x$ to o, $x \in U$, and a family of $\eta$-Whitney coverings $\mathcal{W}_{s}, s>0$. Then there exist $\omega \geq 0$ and $A_{1} \geq 1$ such that $\psi$ is $\left(\omega, A_{1}\right)$-controlled on $U$. Here $A_{1}=A^{2+4 \kappa}$ and $\omega=2 \kappa \log _{2} A$ with $\kappa=D^{4+\log _{2}(1+1 /(\alpha \eta))}$.

Proof: Using the notation of Definition 5.9, we need to compare the values taken by the weight $\psi$ at any pair of points $x_{0}, x_{i}$, such that $x_{0}$ is the center of a Whitney ball $E$ and $x_{i}$ is the center of a ball belonging to the local s-chain $\mathcal{W}_{<s}^{\prime}(E)$. This local s-chain is made of balls in $\mathcal{W}_{s}^{\prime}$, each of which has radius at most $3 s$ and intersects the John path $\gamma_{E}=\gamma_{x_{0}}$ joining $x_{0}$ to $o$.

Assume that we can prove that

$$
\#\left\{K \in \mathcal{W}_{<s}: 2 K \cap \gamma_{E} \neq \emptyset\right\} \leq \kappa \log _{2}(4 s) .
$$

Of course, under this assumption,

$$
1+q_{s}^{*}(E)=\# \mathcal{W}_{<s}^{\prime}(E) \leq 1+\kappa \log _{2}(4 s) .
$$

Further, by definition of $\mathcal{W}_{<s}^{\prime}(E)=\left(F_{0}^{s, E}, \ldots, F_{q_{s}^{*}(E)}^{s, E}\right)$, the balls $2 F_{i}^{s, E}, 2 F_{i+1}^{s, E}$ have a non-empty intersection or are singletons $\left\{x_{i}\right\},\left\{x_{i+1}\right\}$ with $\left\{x_{i}, x_{i+1}\right\} \in \mathfrak{E}$. Since $\psi$ is $(\eta, A)$-regular and the ball $2 F_{i}^{s, E}$ has radius $6 r\left(x_{i}\right) \leq 3 \eta \delta\left(x_{i}\right) / 2$, we have

$$
\psi\left(x_{i}\right) \leq A^{2} \psi\left(x_{i+1}\right), \quad i=0, \ldots, q_{s}^{*}(E) .
$$

This implies

$$
\psi\left(x_{0}\right) \leq A^{2\left(1+\kappa \log _{2}(4 s)\right)} \psi\left(x_{i}\right)=A^{2+4 \kappa} s^{2 \kappa \log _{2} A} \psi\left(x_{i}\right), \quad i=0, \ldots, q_{s}^{*}(E) .
$$

To prove (5.2), for each $\rho \geq 1$, let the John path $\gamma_{x_{0}}$ be

$$
\gamma_{x_{0}}=\left(\xi_{0}=x_{0}, \ldots, \xi_{m}=o\right) \text {. }
$$

Consider

$$
\#\left\{K=B(x, r) \in \mathcal{W}_{<s}: 3 K \cap \gamma_{E} \neq \emptyset, r \in[\rho, 2 \rho)\right\}, \quad \rho \geq 1 .
$$

Let $K=B(x, r), K^{\prime}=B\left(x^{\prime}, r^{\prime}\right)$ be any two balls from that set and let $\xi_{i} \in 3 K$ and $\xi_{i}^{\prime} \in 3 K^{\prime}$ be two points on the John path $\gamma_{x_{0}}$ that are witness to the fact that these balls intersect $\gamma_{x_{0}}$. Now, by construction,

$$
d\left(x, \xi_{i}\right) \leq 3 r, r=\eta \delta(x) / 4 \text { and } \delta\left(\xi_{i}\right) \geq \alpha(1+i)
$$

It follows by the triangle inequality that $\delta(x) \geq \delta\left(\xi_{i}\right)-(3 \eta / 4) \delta(x)$ and thus, using a similar argument for $x^{\prime}, \xi_{i}^{\prime}, r^{\prime}$,

$$
\delta(x) \geq(\alpha / 2)(1+i) \text { and } \delta\left(x^{\prime}\right) \geq(\alpha / 2)\left(1+i^{\prime}\right) .
$$

This, combined with the assumption that $r \in[\rho, 2 \rho)$ from (5.4), implies that $1+$ $\max \{i, j\} \leq(16 / \alpha \eta) \rho$ and

$$
d\left(x, x^{\prime}\right) \leq 8\left(1+\frac{2}{\alpha \eta}\right) \rho, B\left(x^{\prime}, \rho\right) \subset B\left(x,\left(9+\frac{16}{\alpha \eta}\right) \rho\right) .
$$


By construction, the balls $K^{\prime} \in \mathcal{W}_{<s}$ are disjoint and the doubling property of $\pi$ thus implies that

$$
\#\left\{K=B(x, r) \in \mathcal{W}_{<s}: 3 K \cap \gamma_{E} \neq \emptyset, r \in[\rho, 2 \rho)\right\} \leq D^{4+\log _{2}(1+1 /(\alpha \eta))} .
$$

The same argument shows that

$$
\#\left\{K=B(x, r) \in \mathcal{W}_{<s}: 3 K \cap \gamma_{E} \neq \emptyset, r \in(0,1)\right\} \leq D^{4+\log _{2}(1+1 /(\alpha \eta))} .
$$

If $s \in\left(2^{k}, 2^{k+1}\right]$ for some $k$, this implies

$$
\begin{aligned}
\#\left\{K \in \mathcal{W}_{<s}: 3 K \cap \gamma_{E} \neq \emptyset\right\} \leq D^{4+\log _{2}(1+1 /(\alpha \eta))}(k+2) \\
\leq D^{4+\log _{2}(1+1 /(\alpha \eta))} \log _{2}(4 s) .
\end{aligned}
$$

This validates (5.2), which yields

$$
\psi\left(x_{0}\right) \leq A^{2+4 \kappa} s^{2 \kappa \log _{2} A} \psi\left(x_{i}\right)
$$

for $i=0, \ldots, q_{s}^{*}(E), \kappa=D^{4+\log _{2}(1+1 /(\alpha \eta))}$.

\section{Application to Metropolis-type chains}

6.1. Metropolis-type chains. We are ready to apply the technical results developed so far (primarily within Section 5) to Metropolis-type chains on John domains. The reader may find motivation in the explicit examples of Section 6.3. First we explain what we mean by Metropolis-type chains. Classically, The Metropolis and Metropolis Hastings algorithms give a way of changing the output of one Markov chain to have a desired stationary distribution. See Liu (2008) or Diaconis and Saloff-Coste (1998) for background and examples.

Assume we are given the background structure $(\mathfrak{X}, \mathfrak{E}, \mu, \pi)$ with $\mathfrak{X}$ finite or countable. Assume that $\mu$ is adapted and subordinated to $\pi$. Let $U$ be a finite domain in $\mathfrak{X}$. This data determines an irreducible Markov kernel $K_{N, U}$ on $U$ with reversible probability measure $\pi_{U}$, proportional to $\left.\pi\right|_{U}$, given by (this is similar to (3.1))

$$
K_{N, U}(x, y)=\left\{\begin{array}{cc}
\mu_{x y} / \pi(x) & \text { for } x \neq y, x, y \in U \\
1-\left(\sum_{z \in U: z \sim x} \mu_{x z} / \pi(x)\right) & \text { for } x=y \in U .
\end{array}\right.
$$

The notation $K_{N, U}$ captures the idea that this kernel corresponds to imposing the Neumann boundary condition in $U$ (i.e., some sort of reflection of the process at the boundary).

Suppose now that we are given a vertex weight $\psi$ and a symmetric edge weight $h_{x y}$ on the domain $U$. Set

$$
\tilde{\pi}=\psi \pi, \quad \tilde{\mu}_{x y}=\mu_{x y} h_{x y}
$$

and assume that

$$
\sum_{y \in U} \widetilde{\mu}_{x y} \leq \tilde{\pi}
$$

so that $\widetilde{\mu}$ is subordinated to $\widetilde{\pi}$ in $U$. This yields a new Markov kernel $\widetilde{K}$ defined on $U$ by

$$
\widetilde{K}(x, y)=\left\{\begin{array}{cc}
\widetilde{\mu}_{x y} / \widetilde{\pi}(x) & \text { for } x \neq y, x, y \in U \\
1-\left(\sum_{z \in U: z \sim x} \widetilde{\mu}_{x z} / \widetilde{\pi}(x)\right) & \text { for } x=y \in U .
\end{array}\right.
$$

This kernel is irreducible and reversible with reversible probability measure proportional to $\widetilde{\pi}$. 
Example 6.1. The choice $h_{x y}=\min \{\psi(x), \psi(y)\}$ satisfies this property and yields the well-known Metropolis chain with proposal chain $\left(K_{N, U}, \pi_{U}\right)$ and target probability measure $\widetilde{\pi}_{U}$, proportional to $\widetilde{\pi}=\left.\psi \pi\right|_{U}$. Other choice of $h$ would lead to similar chains including the variants of the Metropolis algorithm considered by Hastings and Barker. See the discussion in Billera and Diaconis (2001, Remark $3.1)$.

6.2. Results for Metropolis type chains. In order to simplify notation, we fix the background structure $(\mathfrak{X}, \mathfrak{E}, \pi, \mu)$. We assume that $\pi$ is $D$-doubling, $\mu$ is adapted and that the pair $(\pi, \mu)$ is elliptic with ellipticity constant $P_{e}$ and satisfies the $\theta$-Poincaré inequality on balls with constant $P$. We also assume that $\mu$ is subordinated to $\pi$. We also fix $\alpha \in(0,1)$. In the statements below, we will use $c, C$ to denote quantities whose exact values change from place to place and depend only on $\theta, D, P_{e}, P$ and $\alpha$. Explicit descriptions of these quantitates in terms of the data can be obtained from the proofs. They are of the form

$$
\max \left\{c^{-1}, C\right\} \leq A_{1}^{\theta} D^{A_{2}(1+\log 1 / \alpha)} \max \left\{P_{e}, P\right\}
$$

where $A_{1}, A_{2}$ are universal constants.

Within this fixed background, we consider the collection of all finite domains $U \subset \mathfrak{X}$ which are John domains of type $J(\alpha, o, R)$ for some point $o \in U$ and $R \leq 2 R(U, o, \alpha)$. The parameter $R$ is allowed to vary freely and all estimates are expressed in terms of $R$. Recall that $\rho_{o}(U)=\max \left\{d_{U}(o, x): x \in U\right\}$ satisfies

$$
2 R(U, o, \alpha) \geq 2 \rho_{o}(U) \geq \delta(o) \geq \alpha R(U, o, \alpha) .
$$

We always assume implicitly that $U$ is not reduced to a singleton so that $R(U, o, \alpha) \geq$ 1. Since $\alpha$ is fixed, it follows that $R \asymp \rho_{o}(U)$, namely,

$$
\frac{\alpha}{2} R \leq \rho_{o}(U) \leq 4 R
$$

We need the following simple technical lemma.

Lemma 6.2. Assume that $U \in J(o, \alpha, R)$, with $R \leq 2 R(U, o, \alpha)$, is not a singleton and $0<\eta<1 / 4$. Referring to the construction of the ball $F(s, x), s>0, x \in U$, used in Definition 4.7, any $\eta$-Whitney covering $\mathcal{W}_{s}$ of $U$ satisfies

- $W_{=s}=\emptyset$ whenever $s \geq 3 R(U, o, \alpha)$. In that case, $F(s, x)=3 E_{o}^{s}$ for all $x \in U$ and the ball $E_{o}^{s}$ has radius

$$
r(o)=\eta \delta(o) / 4 \text { with } \alpha R(U, o, \alpha) \leq \delta(o) \leq 2 R(U, o, \alpha) .
$$

- When $s \leq \alpha \eta R(U, o, \alpha) / 4$, all balls $F(s, x)$ have radius $3 s$.

- When $s \in(\alpha \eta R(U, o, \alpha) / 4,3 R(U, o, \alpha))$, each ball $F(s, x), x \in U$, has radius contained in the interval

$$
[\alpha \eta R(U, o, \alpha) / 2,9 R(u, o, \alpha)] .
$$

In particular, for all $s \in(0, \alpha \eta R / 8)$

$$
\frac{\pi(F(s, x))}{\pi(U)} \geq \frac{\pi(B(z(x), s))}{\pi(B(z(x), 8 R))} \geq \frac{1}{D^{2}}\left(\frac{1+s}{8 R}\right)^{\log _{2} D},
$$

and, for all $s \in(0, \alpha \eta R / 8)$, the averaging operator $Q_{s}$ (Definition 4.7) satisfies

$$
\left\|Q_{s} f\right\|_{\infty} \leq M(1+s)^{-\log _{2} D}\|f\|_{1}
$$

where $\|f\|_{1}=\sum_{x \in U}|f(x)| \pi(x)$ and $M=D^{2}(8 R)^{\log _{2} D} / \pi(U)$. 
Remark 6.3. The bound (6.3) is a version of moderate growth for the metric measure space $\left(U, d_{U}, \pi\right)$ with the additional twist that, for each $s, x$, we consider the ball $F(s, x)$ instead of the ball $B_{U}(x, s)$. The reason for this is that it is the balls $F(s, x)$ that appear in the definition of the operator $Q_{s}$ because of the crucial use we make of the Whitney coverings $\mathcal{W}_{s}, s>0$.

Our first result concerns the Markov chain driven by $K_{N, U}$ defined in Example 6.1. This is a reversible chain with reversible probability measure $\pi_{U}$. We let $\beta=\beta_{N, U}$ be the second largest eigenvalue of $K_{N, U}$ and $\beta_{-}=\beta_{N, U,-}$ be the smallest eigenvalue of $K_{N, U}$. From the definition, it is possible that $U=\mathfrak{X}$ and $\beta_{-}=-1$.

Theorem 6.4. There exist constants $c, C$ such that for any $R>0$ and any finite domain $U \in J(\alpha, R)$, we have

$$
1-\beta_{N, U} \geq c R^{-\theta} \text {. }
$$

Assume further that $1+\beta_{N, U,-} \geq c R^{-\theta}$. Under this assumption, for all $t \geq R^{\theta}$,

$$
\max _{x, y \in U}\left\{\left|\frac{K_{N, U}^{t}(x, y)}{\pi_{U}(y)}-1\right|\right\} \leq C e^{-2 c t / R^{\theta}} .
$$

Proof: This result is a consequence of Theorems 4.6 and 4.10. We use a Whitney covering family $W_{s}, s>0$, with $\eta=1 / 4$. For later purpose when we will need to use a given $\eta$, we keep $\eta$ as a parameter in the proof. Theorem 4.6 gives the estimates for $1-\beta_{N, U}$. (Theorem 4.10 also gives that eigenvalue estimate if we pick $s \asymp R$ large enough that the Whitney covering $\mathcal{W}_{s}$ is such that $W_{=s}$ is empty.) By Lemma 6.2 , for $s \in(0, \alpha \eta R / 8]$

$$
\left\|Q_{s} f\right\|_{\infty} \leq M(1+s)^{-\log _{2} D}\|f\|_{1}
$$

where $M=D^{2}(8 R)^{\log _{2} D} / \pi(U)$. Now, we appeal to Theorem 4.10 and Propositions 3.12 and 3.14 to obtain

$$
\sup _{x, y \in U}\left\{K^{t}(x, y) / \pi(y)\right\} \leq C \pi(U)^{-1}\left(R^{\theta} /(n+1)\right)^{\log _{2} D / \theta}
$$

for all $t \leq(\alpha \eta R / 4)^{\theta}$. This is the same as

$$
\sup _{x, y \in U}\left\{K^{t}(x, y) / \pi_{U}(y)\right\} \leq C\left(R^{\theta} /(n+1)\right)^{\log _{2} D / \theta}
$$

for all $t \leq(\alpha \eta R / 4)^{\theta}$, because $\pi_{U}=\left.\pi(U)^{-1} \pi\right|_{U}$. The constant $C$ is of the type described above and incorporates various factors depending only on $D, \theta, \alpha, P, P_{e}$ which are made explicit in Theorem 4.10, Lemma 6.2 and Propositions 3.12 and 3.14.

The next step is (essentially) Diaconis and Saloff-Coste (1996, Lemma 1.1). Using operator notation for ease, write

$$
\sup _{x, y \in U}\left\{\left|\frac{K^{t}(x, y)}{\pi_{U}(y)}-1\right|\right\}=\left\|\left(K-\pi_{U}\right)^{t}\right\|_{L^{1}\left(\pi_{U}\right) \rightarrow L^{\infty}}
$$

and observe that, for any $t_{1}, t_{2}$ such that $t=t_{1}+2 t_{2},\left\|\left(K-\pi_{U}\right)^{t}\right\|_{L^{1}\left(\pi_{U}\right) \rightarrow L^{\infty}}$ is bounded above by the product of

$$
\left\|\left(K-\pi_{U}\right)^{t_{2}}\right\|_{L^{1}\left(\pi_{U}\right) \rightarrow L^{2}\left(\pi_{U}\right)}, \quad\left\|\left(K-\pi_{U}\right)^{t_{1}}\right\|_{L^{2}\left(\pi_{U}\right) \rightarrow L^{2}\left(\pi_{U}\right)}
$$

and

$$
\left\|\left(K-\pi_{U}\right)^{t_{2}}\right\|_{L^{2}\left(\pi_{U}\right) \rightarrow L^{\infty}}
$$


The first and last factors are equal (reversibility and duality) and

$$
\left\|\left(K-\pi_{U}\right)^{t_{2}}\right\|_{L^{2}\left(\pi_{U}\right) \rightarrow L^{\infty}}^{2}=\sup _{x, y \in U}\left\{\left|\frac{K^{2 t_{2}}(x, y)}{\pi_{U}(y)}-1\right|\right\} .
$$

For a proof of this fact, see Saloff-Coste (1997). The second factor is

$$
\left\|\left(K-\pi_{U}\right)^{t_{2}}\right\|_{L^{2}\left(\pi_{U}\right) \rightarrow L^{2}\left(\pi_{U}\right)}=\max \left\{\beta_{N, U},\left|\beta_{N, U,-}\right|\right\}^{t_{1}}
$$

We pick $t_{2}$ to be the largest integer less than or equal to $(\alpha \eta R)^{\theta} /\left(2 \cdot 4^{\theta}\right)$ and apply (6.4) to obtain

$$
\sup _{x, y \in U}\left\{\left|\frac{K^{t}(x, y)}{\pi_{U}(y)}-1\right|\right\} \leq 2^{\log _{2} D / \theta} C(\alpha \eta)^{\log _{2} D / \theta} \max \left\{\beta_{N, U},\left|\beta_{N, U,-}\right|\right\}^{t_{1}} .
$$

This gives the desired result.

The following very general example illustrates the previous theorem.

Example 6.5 (Graph metric balls). Fix constants $P_{e}, P, \theta$ and $D$. Assume that $(\mathfrak{X}, \mathfrak{E}, \pi, \mu)$ is such that the volume doubling property holds with constant $D$ together with $P_{e}$-ellipticity and the $\theta$-Poincaré inequality with constant $P$. We also assume (for simplicity) that

$$
\sum_{y \sim x} \mu_{x y} \leq \pi(x) / 2 .
$$

Under this assumption, for any finite domain $U$, the kernel $K_{N, U}$ has the property that $K_{N, U}(x, x) \geq 1 / 2$ (this is often called "laziness") and it implies that $\beta_{N, U,-} \geq 0$.

Let $U=B(o, R)$ be any graph metric ball in $(\mathfrak{X}, \mathfrak{E})$. From Example 2.10, such a ball is a John domain with $\alpha=1$, namely, $U \in J(\mathfrak{X}, \mathfrak{E}, 1, o, R)$ and $R=R(U, \alpha, o)$. Since $\beta_{N, U,-} \geq 0$, Theorem 6.4 applies and show that $K_{N, U}^{t}$ converges to $\pi_{U}$ in times of order $R^{\theta}$. This applies for instance to the metric balls of the Vicsek graph of Figure 3.10.

Next we consider a weight $\psi$ which is $(\eta, A)$-regular to $U$ and $A$-doubling. This means that the measure $\widetilde{\pi}=\psi \pi$ is $A$ doubling on $\left(U, \mathfrak{E}_{U}\right.$ ) (and also, by extension, $\left(\mathfrak{U}, \mathfrak{E}_{\mathfrak{U}}\right)$ is $2 A$ doubling). For simplicity we pick $\widetilde{\mu}$ to be given by the Metropolis choice

$$
\widetilde{\mu}_{x y}=\mu_{x y} \min \{\tilde{\pi}(x), \tilde{\pi}(y)\}
$$

This implies that $\widetilde{\mu}$ is subordinated to $\widetilde{\pi}$ and we let

$$
K_{U, \psi}=\widetilde{K}
$$

be defined by (6.2). This reversible Markov kernel has reversible probability measure $\tilde{\pi}_{U}$ proportional to $\psi \pi$ on $U$. Also, the hypothesis that $\psi$ is $(\eta, A)$-regular to $U$ implies that the pair $(\widetilde{\psi}, \widetilde{\mu})\left(\eta, A^{2}\right)$-dominates $(\pi, \mu)$ on $U$. See Remark 5.8. This shows that we can use Theorem 5.5 to prove the following result using the same line of reasoning as for Theorem 6.4. We will denote by $\beta_{U, \psi}$ the second largest eigenvalue of $\widetilde{K}=K_{U, \psi}$ and by $\beta_{U, \psi,-}$ its lowest eigenvalue.

Theorem 6.6. For fixed $\eta \in(0,1 / 8), A \geq 1$, there exist constants $c, C$ such that for any $R>0$, any finite domain $U \in J(\alpha, R)$ and any weight $\psi$ which is $(\eta, A)$-regular (see Definition 5.7) and A-doubling on $U$, we have

$$
1-\beta_{U, \psi} \geq c R^{-\theta} \text {. }
$$


Assume further that $1+\beta_{U, \psi,-} \geq c R^{-\theta}$. Under this assumption, for all $t \geq R^{\theta}$,

$$
\max _{x, y \in U}\left\{\left|\frac{\widetilde{K}^{t}(x, y)}{\widetilde{\pi}_{U}(y)}-1\right|\right\} \leq C e^{-2 c t / R^{\theta}} .
$$

There are universal constants $A_{1}, A_{2}$ such that

$$
\max \left\{c^{-1}, C\right\} \leq A_{1}^{\theta}(A D)^{A_{2}(1+\log 1 / \alpha \eta)} \max \left\{P_{e}, P\right\} .
$$

Replacing the hypothesis that $\psi$ is $(\eta, A)$-regular and $A$-doubling by the hypothesis that $\psi$ is $(\eta, A)$-regular and $(\omega, A)$-controlled leads to the following similar statement.

Theorem 6.7. For fixed $\eta \in(0,1 / 8), A \geq 1$ and $\omega \geq 0$, there exist constants $c, C$ such that for any $R>0$, any finite domain $U \in J(\alpha, R)$ and any weight $\psi$ which is $(\eta, A)$-regular and $(\omega, A)$-controlled (see Definition 5.9) on $U$, we have

$$
1-\beta_{U, \psi} \geq c R^{-(\theta+\omega)}
$$

Assume further that $1+\beta_{U, \psi,-} \geq c R^{-(\theta+\omega)}$. Under this assumption, for all $t \geq R^{\theta}$,

$$
\max _{x, y \in U}\left\{\left|\frac{\widetilde{K}^{t}(x, y)}{\widetilde{\pi}_{U}(y)}-1\right|\right\} \leq C e^{-2 c t / R^{(\theta+\omega)}} .
$$

There are universal constants $A_{1}, A_{2}$ such that

$$
\max \left\{c^{-1}, C\right\} \leq A_{1}^{\theta+\omega}(A D)^{A_{2}(1+\log 1 / \alpha \eta)} \max \left\{P_{e}, P\right\} .
$$

6.3. Explicit examples of Metropolis type chains. We give four simple and instructive explicit examples regarding Metropolis chains. There are based on a cube $U=[-N, N]^{d}$ in some fixed dimension $d$. The key parameter which is allowed to vary is $N$. This cube is equipped with its natural edge structure induced by the integer lattice. The underlying edge weight is $\mu_{x, y}=(2 d)^{-1}$ and $\pi$ is the counting measure.

To obtain each of our examples, we will define a "boundary" for $U$ and a weight $\psi$ that is $(1 / 8, A)$-regular and $A$ doubling.

Example 6.8. Our first example uses the natural boundary of $U=[-N, N]^{d}$ in the square grid $\mathbb{Z}^{d}$. The weight $\psi=\psi_{\nu}, \nu \geq 0$, is given by

$$
\psi(x)=\delta(x)^{\nu} .
$$

Recall that $\delta(x)$ is the distance to the boundary. Thus, this power weight is largest at the center of the cube. It is $(1 / 8, A)$-regular and $A$-doubling with $A$ depending of $d$ and $\nu$ which we assume are fixed. Theorem 6.6 applies (with $\theta=2$ ). The necessary estimates on the lowest eigenvalue $\beta_{U, \psi,-}$ holds true because there is sufficient holding probability provided by the Metropolis rule at each vertex (this holding is of order at least $1 / N$ and, in addition, there is also enough holding at the boundary). Here $R \asymp N$ and convergence occurs in order $N^{2}$ steps.

Example 6.9. Our second example is obtained by adding two points to the box from the first example, which will serve as the boundary. Let $\mathfrak{X}=[-N, N]^{d} \cup$ $\left\{u_{-}, u_{+}\right\}$, where $u_{-}$is attached by one edge to $(-N, \ldots,-N)$ and $u_{+}$attached by one edge to $(N, \ldots, N)$. Within $\mathfrak{X}$, let $U=[-N, N]^{d}$, so the boundary is $\left\{u_{-}, u_{+}\right\}$. Again, we consider the power weight

$$
\psi(x)=\psi_{\nu}(x)=\delta(x)^{\nu}, \quad \nu>0
$$


but this time $\delta$ is the distance to the boundary $\left\{u_{-}, u_{+}\right\}$. This power weight is constant along the hyperplanes $\sum_{i=1}^{d} x_{i}=k$ and maximum on $\sum_{i=1}^{d} x_{i}=0$.

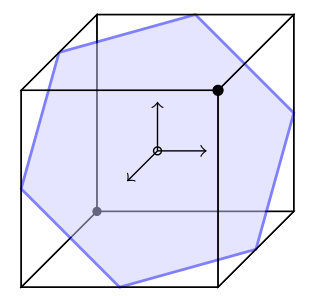

Figure 6.12 . The box $U=[-N, N]^{3}$ with two boundary points $u_{1}, u_{+}$attached at corners $(-N,-N,-N)$ and $(N, N, N)$ (these to corners are marked with black dots). The blue plane is the set of points in $U$ at maximal distance from the boundary points $\left\{u_{-}, u_{+}\right\}$. The center of the box is shown with the axes. The grid is not shown.

This weight is $(1 / 8, A)$-regular and $A$-doubling with $A$ depending of $d$ and $\nu$ which are fixed. Theorem 6.6 applies (with $\theta=2$ ). The necessary estimates on the lowest eigenvalue $\beta_{U, \psi,-}$ hold true because there is sufficient holding probability provided by the Metropolis rule (again, at least order $1 / N$ at each vertex). We have $R \asymp N$ and convergence occurs in order $N^{2}$ steps.

Example 6.10. Our third example is obtained by adding only one boundary point to the box from the first example. Let $\mathfrak{X}=[-N, N]^{d} \cup\left\{u_{0}\right\}$ where $u_{0}$ is attached by one edge to the center $(0, \ldots, 0)$. Within $\mathfrak{X}$, let $U=[-N, N]^{d}$, so the boundary is $\left\{u_{0}\right\}$. Still, we consider the power weight

$$
\psi(x)=\psi_{\nu}(x)=\delta(x)^{\nu}, \nu>0,
$$

where $\delta$ is the distance to the boundary $\left\{u_{0}\right\}$. This power weight is constant along the boundary of the graph balls centered at $(0, \ldots, 0)$. It is largest at the $2 d$ corners. In this case, we obtain a John domain with a fixed $\alpha$ only when $d>1$ (in the case $d=1$, there is no way to avoid passing near the boundary point $\left.u_{0}\right)$. When $d>1$, we can chose $o$ to be one of the four corners. Again, the weight is $(1 / 8, A)$-regular and $A$ doubling with $A$ depending on $d$ and $\nu$ which are fixed. Theorem 6.6 applies (with $\theta=2$ ). The necessary estimates on the lowest eigenvalue $\beta_{U, \psi,-}$ hold true as in the previous examples. Again, $R \asymp N$ and convergence occurs in order $N^{2}$ steps. We note that there is no problems replacing the single "pole" 0 in this example by an arbitrary finite set $\mathfrak{O}$ of "poles", as long as we fix the number of elements in $\mathfrak{O}$.

Example 6.11. This last example involves weights which lead to non-doubling measure but are $\omega$-controlled. Take $d=1$ and $U=[-N, \ldots, N]$, a symmetric interval around 0 in $\mathbb{Z}$. Fix $\nu>1$ and consider the weight $\psi_{\nu}=\delta(x)^{-\nu}$, where $\delta$ is the distance to the boundary $\{-N-1, N+1\}$. It is easy to check that this weight is not doubling (compare the $\widetilde{\pi}$-volume of $B(0, N / 2)$ to that of $B(0, N))$. Obviously, $\psi_{\nu}$ is $\omega$-controlled with $\omega=\nu$. The reference Saloff-Coste (1999, Theorem 9.6) applies to this family and provides the eigenvalue estimate

$$
1-\beta_{U, \psi_{\nu}} \approx N^{-1-\nu}
$$


and the fact that this chain converges to its equilibrium measure in order $N^{1+\nu}$ steps. This should be compared with the eigenvalue estimate of Theorem 6.7 which reads $1-\beta_{U, \psi_{\nu}} \geq c N^{-2-\nu}$ because $R \approx N$ and $\omega=\nu$. This estimate is off by a factor of $N$, but it is clear that the parameter $\omega=\nu$ plays a key role in estimating $\beta_{U, \psi_{\nu}}$ in this case. The following modification of this example shows that the eigenvalue estimate of Theorem 6.7 is actually almost optimal. Consider $[-(N+1),(N+1)]$ equipped with the measure $\pi(x)=(N+2-|x|)^{-\alpha}, \alpha \in(0,1)$ and the usual graph structure induced by $\mathbb{Z}$. This space is doubling and satisfies the Poincaré inequality on balls (this is not obvious, but it can be proved). On this space, let $U=[-N, \ldots, N]$ and repeat the construction above with $\psi_{\nu}(x)=\delta(x)^{-\nu}$, $\nu>1-\alpha$. Now, on this new space, this weight is not doubling but it is $\omega$-controlled with $\omega=\nu$. The previous argument shows that the eigenvalue $\beta_{U, \alpha, \psi_{\nu}}$ satisfies $1-\beta_{U, \alpha, \psi_{\nu}} \approx N^{-1-\alpha-\nu}$ whereas Theorem 6.7 yields $\beta_{U, \alpha, \psi_{\nu}} \geq c N^{-2-\nu}$. Since $\alpha$ can be chosen as close to 1 as desired, Theorem 6.7 is indeed almost sharp.

\section{The Dirichlet-type chain in $U$}

We continue with our general setup described by the data $(\mathfrak{X}, \mathfrak{E}, \pi, \mu)$. We assume that $\mu$ is adapted and that $\mu$ is subordinated to $\pi$. For any finite domain $U$, we consider $K_{D, U}$, the Dirichlet-type kernel in $U$, defined by

$$
K_{D, U}(x, y)=\left\{\begin{array}{cc}
\mu_{x y} / \pi(x) & \text { for } x \neq y \text { with } x, y \in U \\
1-\left(\sum_{z \in \mathfrak{X}: z \sim x} \mu_{x z} / \pi(x)\right) & \text { for } x=y \in U .
\end{array}\right.
$$

This is the kernel describing the chain that is killed when it exits $U$. Let us point out the subtle but essential difference between this definition and that of $K_{N, U}$, the Neumann-type kernel on $U$. The values of these two kernels are the same when $x \neq y$ or when $x=y$ has no neighbors outside $U$. But when $x=y$ has a neighbor outside $U$, we have

$$
K_{N, U}(x, x)=1-\left(\sum_{z \in U: z \sim x} \mu_{x z}\right) / \pi(x)
$$

whereas

$$
K_{D, U}(x, x)=1-\left(\sum_{z \in \mathfrak{X}: z \sim x} \mu_{x z}\right) / \pi(x) .
$$

Because $\mu$ is adapted, at such a point $x$,

$$
\sum_{y \in U} K_{N, U}(x, y)=1 \text { whereas } \sum_{y \in U} K_{D, U}(x, y)<1 .
$$

In words, the kernel $K_{D, U}$ is not strictly Markovian and the Markov chain corresponding to this kernel includes killing at the boundary. In terms of the global Markov kernel $K=K_{\mu}$ defined on $\mathfrak{X}$ by (3.1), we have

$$
K_{D, U}=\mathbf{1}_{U}(x) K(x, y) \mathbf{1}_{U}(y) .
$$

To simplify notation, we set

$$
K_{U}=K_{D, U}
$$

The goal of this section is to apply the previous results to the study of the iterated kernel $K_{U}^{t}(x, y)$. This will be done using the method of Doob's transform explained in more general terms in the next subsection. 
7.1. The general theory of Doob's transform. For the purpose of this subsection, we simply assume we are given a finite or countable state space $\mathfrak{X}$ equipped with a Markov kernel $K$. We do not assume any reversibility. Fix a finite subset $U$ and consider the restricted kernel

$$
K_{U}(x, y)=\mathbf{1}_{U}(x) K(x, y) \mathbf{1}_{U}(y) .
$$

Throughout this section, we assume that this kernel $K_{U}$ is irreducible on $U$ in the sense that for any $x, y \in U$ there is an integer $t=t(x, y)$ such that $K_{U}^{t}(x, y)>0$. The period $d$ of $K_{U}$ is the greatest common divisor of $\left\{t: K_{U}^{t}(x, x)>0\right\}$. Note that $d$ is independent of the choice of $x \in U$. When $d=1$ (which is referred to as the aperiodic case), there exists an $N$ such that $K_{U}^{N}(x, y)>0$ simultaneously for all $x, y \in U$. We are interested in understanding the behavior of the chain driven by $K$ on $\mathfrak{X}$, started in $U$ and killed at the first exit from $U$. If $\left(X_{t}\right)_{0}^{\infty}$ denotes the chain driven by $K$ on $\mathfrak{X}$ and

$$
\tau=\tau_{U}=\inf \left\{t \geq 0: X_{t} \notin U\right\}
$$

is the first exit time from $U$, we would like to have good approximations for quantities such as

$$
\mathbf{P}_{x}\left(\tau_{U}>\ell\right), \quad \mathbf{P}_{x}\left(X_{t}=y \mid \tau_{U}>\ell\right), \quad \mathbf{P}_{x}\left(X_{t}=y \text { and } \tau>t\right),
$$

for $x, y \in U, 0 \leq t \leq \ell$. The last of these quantities is, of course,

$$
\mathbf{P}_{x}\left(X_{t}=y \text { and } \tau>t\right)=K_{U}^{t}(x, y) \text {. }
$$

See Collet et al. (2013) for a book length discussion of such problems. The key lemma is the following.

Lemma 7.1. Assume that $K_{U}$ is irreducible and aperiodic. Let $\beta_{0}, \phi_{0}$ denote the Perron-Frobenius eigenvalue and right eigenfunction of $K_{U}$, respectively. The limit

$$
\mathbf{P}_{x}\left(X_{t}=y \mid \tau_{U}=\infty\right)=\lim _{L \rightarrow \infty} \mathbf{P}_{x}\left(X_{t}=y \mid \tau_{U}>L\right)
$$

exists and it is equal to $K_{\phi_{0}}^{t}(x, y)$ where $K_{\phi_{0}}$ is the irreducible aperiodic Markov kernel given by

$$
K_{\phi_{0}}(x, y)=\beta_{0}^{-1} \frac{1}{\phi_{0}(x)} K_{U}(x, y) \phi_{0}(y), \quad x, y \in U
$$

Remark 7.2. When $K_{U}$ is irreducible but periodic, it still has a unique PerronFrobenius eigenvalue and right eigenfunction, $\beta_{0}, \phi_{0}$, and one can still define the Markov kernel $K_{\phi_{0}}$ (and use it to study $K_{U}$ ), but the limit in the lemma does not typically exist. See Example 7.6 below.

Remark 7.3. In general terms, Doob's transform method studies the Markov kernel $K_{\phi_{0}}$ in order to study the iterated kernel $K_{U}^{t}$. By definition,

$$
K_{U}^{t}(x, y)=\beta_{0}^{t} \phi_{0}(x) K_{\phi_{0}}^{t}(x, y) \frac{1}{\phi_{0}(y)} .
$$

Let $\phi_{0}^{*}$ denote the (positive) left eigenfunction of $K_{U}$ associated with $\beta_{0}$. By inspection, the positive function $\phi_{0}^{*} \phi_{0}$, understood as a measure on $U$, is invariant under the action of $K_{\phi_{0}}$, that is,

$$
\sum_{x \in U} \phi_{0}^{*}(x) \phi_{0}(x) K_{\phi_{0}}(x, y)=\phi_{0}^{*}(y) \phi_{0}(y) .
$$


This measure can be normalized to provide the invariant probability measure for the irreducible Markov kernel $K_{\phi_{0}}$. We call this invariant probability measure $\pi_{\phi_{0}}$. It is given by

$$
\pi_{\phi_{0}}(x)=\frac{\phi_{0}^{*}(x) \phi_{0}(x)}{\sum_{y \in U} \phi_{0}^{*}(y) \phi_{0}(y)} .
$$

The measure $\pi_{\phi_{0}}$ is one version of the quasi-stationary distribution (a second version is in Definition 7.7 below). The measure $\pi_{\phi_{0}}$ gives the limiting behavior of the chain, conditioned never to be absorbed. As shown below, it is the key to understanding the absorbing chain as well. The Doob transform is a classical tool in Markov chain theory Kemeny et al. (1976, Chapter 8). For many applications and a literature review see Pang (2019).

Proof of Lemma \%.1: Fix $T \in \mathbb{N}$ and any $t \leq T$. For each $L>0$, we have

$$
\begin{aligned}
& \mathbf{P}_{x}\left(X_{t}=y, \tau_{U}>t \mid \tau_{U}>L\right) \\
& \quad=\frac{\mathbf{P}_{x}\left(\tau_{U}>L \mid X_{t}=y, \tau_{U}>t\right) \mathbf{P}_{x}\left(X_{t}=y, \tau_{U}>t\right)}{\mathbf{P}_{x}\left(\tau_{U}>L, \tau_{U}>t\right)}
\end{aligned}
$$

We can assume $L>T$, because we will later take the limit as $L$ tends to infinity. So (7.4), the identity above, becomes,

$$
\begin{aligned}
& \mathbf{P}_{x}\left(X_{t}=y \mid \tau_{U}>L\right) \\
& \quad=\frac{\mathbf{P}_{x}\left(\tau_{U}>L \mid X_{t}=y, \tau_{U}>t\right) \mathbf{P}_{x}\left(X_{t}=y, \tau_{U}>t\right)}{\mathbf{P}_{x}\left(\tau_{U}>L\right)}
\end{aligned}
$$

or equivalently,

$$
\mathbf{P}_{x}\left(X_{t}=y \mid \tau_{U}>L\right)=\frac{\mathbf{P}_{x}\left(\tau_{U}>L \mid X_{t}=y, \tau_{U}>t\right)}{\mathbf{P}_{x}\left(\tau_{U}>L\right)} K_{U}^{t}(x, y)
$$

Because $\left(X_{t}\right)$ is a Markov chain,

$$
\begin{aligned}
\frac{\mathbf{P}_{x}\left(\tau_{U}>L \mid X_{t}=y, \tau_{U}>t\right)}{\mathbf{P}_{x}\left(\tau_{U}>L\right)} & =\frac{\mathbf{P}_{y}\left(\tau_{U}>L-t\right)}{\mathbf{P}_{x}\left(\tau_{U}>L\right)} \\
& =\frac{\sum_{z \in U} K_{U}^{L-t}(y, z)}{\sum_{z \in U} K_{U}^{L}(x, z)} \\
& =\frac{\sum_{z \in U} \beta_{0}^{L-t} \phi_{0}(y) K_{\phi_{0}}^{L-t}(y, z) \phi_{0}(z)^{-1}}{\sum_{z \in U} \beta_{0}^{L} \phi_{0}(x) K_{\phi_{0}}^{L}(x, z) \phi_{0}(z)^{-1}},
\end{aligned}
$$

where the last line follows by (7.3).

Plugging this into (7.5), we have

$$
\begin{aligned}
& \mathbf{P}_{x}\left(X_{t}=y \mid \tau_{U}>L\right)= \\
& \quad=\left[\frac{\sum_{z \in U} K_{\phi_{0}}^{L-t}(y, z) \phi_{0}(z)^{-1}}{\sum_{z \in U} K_{\phi_{0}}^{L}(x, z) \phi_{0}(z)^{-1}}\right] \beta_{0}^{-t} \phi_{0}(x)^{-1} K_{U}^{t}(x, y) \phi_{0}(y)
\end{aligned}
$$

Now we take the limit as $L$ tends to infinity. To finish the proof of Lemma 7.1 we need to show that

$$
\lim _{L \rightarrow \infty} \frac{\sum_{z \in U} K_{\phi_{0}}^{L-t}(y, z) \phi_{0}(z)^{-1}}{\sum_{z \in U} K_{\phi_{0}}^{L}(x, z) \phi_{0}(z)^{-1}}=1
$$

which is the content of the following, Lemma 7.4. 
Lemma 7.4. Assume that $K_{U}(x, y)$ is irreducible and aperiodic on $U$. Then,

$$
\lim _{L \rightarrow \infty} \frac{\sum_{z \in U} K_{\phi_{0}}^{L-t}(y, z) \phi_{0}(z)^{-1}}{\sum_{z \in U} K_{\phi_{0}}^{L}(x, z) \phi_{0}(z)^{-1}}=1 .
$$

Proof: By Remark 7.3, $K_{\phi_{0}}$ is an irreducible aperiodic Markov kernel with invariant measure $\pi_{\phi_{0}}$ proportional to $\phi_{0}^{*} \phi_{0}$. By the basic convergence theorem for finite Markov chains (e.g., Norris, 1998, Thm. 1.8.5),

$$
\lim _{L \rightarrow \infty} K_{\phi_{0}}^{L}(x, y)=\pi_{\phi_{0}}(y) \text {. }
$$

Applying this to

$$
\frac{\sum_{z \in U} K_{\phi_{0}}^{L-t}(y, z) \phi_{0}(z)^{-1}}{\sum_{z \in U} K_{\phi_{0}}^{L}(x, z) \phi_{0}(z)^{-1}},
$$

we can see that both the numerator and denominator approach

$$
\sum_{z \in U} \pi_{\phi_{0}}(z) \phi_{0}(z)^{-1}
$$

The stated result follows.

Remark 7.5. If $K_{U}$ is irreducible and periodic of period $d>1$, then so is $K_{\phi_{0}}$. The chain driven by $K_{\phi_{0}}$ has $d$ periodic classes, $C_{i}$ (with $0 \leq i \leq d-1$ ), each of which has the same measure, $\pi_{\phi_{0}}\left(C_{i}\right)=\pi_{\phi_{0}}\left(C_{0}\right)$, and the limit theorem reads

$$
\lim _{L \rightarrow \infty} K_{\phi_{0}}^{t+L d}(x, y)=\left\{\begin{array}{cl}
\pi_{\phi_{0}}(y) / d & \text { if } x \in C_{i}, y \in C_{i+t} \\
0 & \text { otherwise. }
\end{array}\right.
$$

Here, $0 \leq i \leq d-1$, and the index $i+t$ in $C_{i+t}$ is taken modulo $d$. It follows that, typically, the ratio in Lemma 7.4 has no limit. See below for a concrete example.

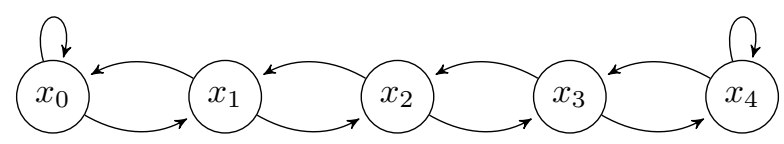

Example 7.6. As a concrete example, consider the simple random walk on five vertices where the boundary vertices have holding probability $\frac{1}{2}$.

$$
K\left(x_{i}, x_{j}\right)=\left\{\begin{array}{ll}
\frac{1}{2} & \text { if }|i-j|=1, i=j=0, \text { or } i=j=4 \\
0 & \text { else }
\end{array} .\right.
$$

Let $U=\left\{x_{1}, x_{2}, x_{3}\right\}$ be the middle three vertices and define $K_{U}$ to be subMarkovian kernel described above. The transition matrix for $K_{U}$ is given by

$$
\left[\begin{array}{ccc}
0 & \frac{1}{2} & 0 \\
\frac{1}{2} & 0 & \frac{1}{2} \\
0 & \frac{1}{2} & 0
\end{array}\right]
$$

with largest eigenvalue $\beta_{0}=\frac{\sqrt{2}}{2}$ and normalized eigenfunction

$$
\phi_{0}=\left[\begin{array}{c}
\frac{1}{2} \\
\frac{\sqrt{2}}{2} \\
\frac{1}{2}
\end{array}\right] \text {. }
$$


This is a reversible situation (hence, $\phi_{0}^{*}=\phi_{0}$ ) and the period is 2 with periodic classes: $C_{0}=\left\{x_{2}\right\}$ and $C_{1}=\left\{x_{1}, x_{3}\right\}$. We have

$$
\lim _{L \rightarrow \infty} \sum_{y \in U} K_{\phi_{0}}^{2 L}\left(x_{2}, y\right) \phi_{0}^{-1}(y)=\sqrt{2}
$$

and

$$
\lim _{L \rightarrow \infty} \sum_{y \in U} K_{\phi_{0}}^{2 L+1}\left(x_{2}, y\right) \phi_{0}^{-1}(y)=2,
$$

and hence the ratio in Lemma 7.4 has no limit.

Previously, we were considering $\mathbf{P}_{x}\left(X_{t}=y \mid \tau_{U}>L\right)$, the probability that the process $\left(X_{t}\right)$ equals $y$ at time $t$ and is still inside $U$ at some other time $L$. Now, we consider the case where $t=L$.

Definition 7.7. Set

$$
\nu_{x}^{t}(y)=\mathbf{P}_{x}\left(X_{t}=y \mid \tau_{U}>t\right), \quad x, y \in U .
$$

This is the second form of quasi-stationary distribution; $\nu_{x}^{t}(y)$ describes the chance that the chain is at $y$ at time $t$ (starting from $x$ ) given that it is still alive.

Theorem 7.8. Assume that $K_{U}$ is irreducible and aperiodic. Then

$$
\lim _{t \rightarrow \infty} \nu_{x}^{t}(y)=\frac{\phi_{0}^{*}(y)}{\sum_{z \in U} \phi_{0}^{*}(z)} .
$$

Proof: Write

$$
\begin{aligned}
\nu_{x}^{t}(y) & =\mathbf{P}_{x}\left(X_{t}=y \mid \tau_{U}>t\right) \\
& =\frac{\mathbf{P}_{x}\left(X_{t}=y, \tau_{U}>t\right)}{\mathbf{P}_{x}\left(\tau_{U}>t\right)} \\
& =\frac{K_{U}^{t}(x, y)}{\sum_{z \in U} K_{U}^{t}(x, z)} \\
& =\frac{\beta_{0}^{t} \phi_{0}(x) K_{\phi_{0}}^{t}(x, y) \phi_{0}(y)^{-1}}{\sum_{z \in U} \beta_{0}^{t} \phi_{0}(x) K_{\phi_{0}}^{t}(x, z) \phi_{0}(z)^{-1}} \\
& =\frac{K_{\phi_{0}}^{t}(x, y) \phi_{0}(y)^{-1}}{\sum_{z \in U} K_{\phi_{0}}^{t}(x, z) \phi_{0}(z)^{-1}} .
\end{aligned}
$$

Taking the limit when $t$ tends to infinity yields

$$
\lim _{t \rightarrow \infty} \nu_{x}^{t}(y)=\frac{\phi_{0}(y)^{-1} \pi_{\phi_{0}}(y)}{\sum_{z \in U} \phi_{0}(z)^{-1} \pi_{\phi_{0}}(z)}=\frac{\phi_{0}^{*}(y)}{\sum_{z} \phi_{0}^{*}(z)} .
$$

This equality follows from the basic Markov chain convergence theorem (Levin and Peres, 2017, Theorem 4.9). The stated result follows since $\pi_{\phi_{0}}$ is proportional to $\phi_{0}^{*} \phi_{0}$.

Theorem 7.9. Assume that $K_{U}$ is irreducible and aperiodic. Then the rate of convergence in

is controlled by that of

$$
\lim _{t \rightarrow \infty} \nu_{x}^{t}(\cdot)=\frac{\phi_{0}^{*}(\cdot)}{\sum_{z \in U} \phi_{0}^{*}(z)}
$$

$$
\lim _{t \rightarrow \infty} K_{\phi_{0}}^{t}(x, \cdot)=\pi_{\phi_{0}}(\cdot) .
$$


More precisely, fix $\epsilon>0$. Assume that $N_{\epsilon}$ is such that, for any $t \geq N_{\epsilon}$ and $y \in U$,

$$
\left|\frac{K_{\phi_{0}}^{t}(x, y)}{\pi_{\phi_{0}}(y)}-1\right|<\epsilon .
$$

Then, for any $t \geq N_{\epsilon}$,

$$
\left|\frac{\left(\sum_{z \in U} \phi_{0}^{*}(z)\right) \nu_{x}^{t}(y)}{\phi_{0}^{*}(y)}-1\right|<\frac{2 \epsilon}{1-\epsilon} .
$$

Proof: For a fixed $\epsilon>0$, let $N_{\epsilon}$ be such that, for $t \geq N_{\epsilon}$ and $z \in U$,

$$
\left|\frac{K_{\phi_{0}}^{t}(x, z)}{\pi_{\phi_{0}}(z)}-1\right|<\epsilon
$$

or equivalently,

$$
\left|K_{\phi_{0}}^{t}(x, z) \phi_{0}^{-1}(z)-c \phi_{0}^{*}(z)\right|<\epsilon c \phi_{0}^{*}(z),
$$

where $c=\left(\sum_{x \in U} \phi_{0}(x) \phi_{0}^{*}(x)\right)^{-1}$ is the normalization constant $\pi_{\phi_{0}}=c \phi_{0} \phi_{0}^{*}$. Summing over all $z \in U$ and applying the triangle inequality,

$$
\left|\frac{\sum_{z \in U} K_{\phi_{0}}^{t}(x, z) \phi_{0}^{-1}(z)}{c \sum_{z \in U} \phi_{0}^{*}(z)}-1\right|<\epsilon
$$

For ease of notation, we abbreviate

$$
\begin{aligned}
a_{t}=K_{\phi_{0}}^{t}(x, y) \phi_{0}(y)^{-1}, & a & =c \phi_{0}^{*}(y), \\
b_{t}=\sum_{z \in U} K_{\phi_{0}}^{t}(x, z) \phi_{0}(z)^{-1}, & b & =c \sum_{z \in U} \phi_{0}^{*}(z),
\end{aligned}
$$

so that (7.10) and (7.11) become,

$$
\left|\frac{a_{t}}{a}-1\right|<\epsilon \text { and }\left|\frac{b_{t}}{b}-1\right|<\epsilon
$$

The formula (7.9) for $\nu_{x}^{t}(y)$ gives

$$
\frac{\left(\sum_{w \in U} \phi_{0}^{*}(w)\right) \nu_{x}^{t}(y)}{\phi_{0}^{*}(y)}=\frac{\left(\sum_{U} \phi_{0}^{*}\right) K_{\phi_{0}}^{t}(x, y) \phi_{0}(y)^{-1}}{\phi_{0}^{*}(y) \sum_{z \in U} K_{\phi_{0}}^{t}(x, z) \phi_{0}(z)^{-1}}=\frac{a_{t}}{b_{t}} \cdot \frac{b}{a}
$$

and thus

$$
\begin{aligned}
\left|\frac{\left(\sum_{w \in U} \phi_{0}^{*}(w)\right) \nu_{x}^{t}(y)}{\phi_{0}^{*}(y)}-1\right| & =\left|\frac{a_{t}}{b_{t}} \cdot \frac{b}{a}-1\right|=\left|\frac{a_{t} b-b_{t} a}{b_{t} a}\right| \\
& \leq \frac{b}{b_{t}}\left(\left|\frac{a_{t}}{a}-1\right|+\left|\frac{b_{t}}{b}-1\right|\right) \\
& <\frac{2 \epsilon}{1-\epsilon} .
\end{aligned}
$$


7.2. Dirichlet-type chains in John domains. We return to our main setting of an underlying space $(\mathfrak{X}, \mathfrak{E}, \pi, \mu)$ with $\mu$ subordinated to $\pi$ and $K$ defined by this data as in (3.1). For any finite domain $U \subset \mathfrak{X}$, we consider the kernel $K_{U}=K_{D, U}$ defined at (7.1) and equal to $K_{U}(x, y)=\mathbf{1}_{U}(x) K(x, y) \mathbf{1}_{U}(y)$. We also let $\pi_{U}$ be the probability measure proportional to $\left.\pi\right|_{U}$, i.e., $\pi_{U}(x)=\frac{\left.\pi\right|_{U}(x)}{Z}$ where $Z=$ $\left.\sum_{y \in U} \pi\right|_{U}(y)$ is the normalizing constant. Let $\phi_{0}, \phi_{0}^{*}$ be the right and left PerronFrobenius eigenfunctions of the kernel $K_{U}$ considered in subsection 7.1 above. By construction, $K_{U}(x, y) / \pi_{U}(y)$ is symmetric in $x, y$, that is,

$$
\pi_{U}(y) K_{U}(y, x)=\pi_{U}(x) K_{U}(x, y) .
$$

Multiplying by $\phi_{0}(y)$ and summing over $y$, we have

$$
\sum_{y \in U} \phi_{0}(y) \pi_{U}(y) K_{U}(y, x)=\pi_{U}(x) \sum_{y \in U} K_{U}(x, y) \phi_{0}(y)=\beta_{0} \pi_{U}(x) \phi_{0}(x) .
$$

This shows that $\phi_{0}(y) \pi_{U}(y)$ is proportional to $\phi_{0}^{*}(y)$. If we choose to normalize $\phi_{0}$ by the natural condition $\sum x \in U \phi_{0}^{2}(x) \pi_{U}(x)=1$, then the invariant probability measure of the Doob transform kernel $K_{\phi_{0}}$ at (7.2) - which is proportional to $\phi_{0}^{*} \phi_{0}-$ is

$$
\pi_{\phi_{0}}=\phi_{0}^{2} \pi_{U}
$$

Next, observe that, for any $x, y \in \mathfrak{X}$,

$$
\pi(x) K(x, y)=\mu_{x y}
$$

and, for any $x, y \in U$,

$$
\left.\phi_{0}^{2}(x) \pi\right|_{U}(x) K_{\phi_{0}}(x, y)=\left.\beta_{0}^{-1} \phi_{0}(x) \phi_{0}(y) \pi\right|_{U}(x) K(x, y)=\beta_{0}^{-1} \phi_{0}(x) \phi_{0}(y) \mu_{x y} .
$$

This means that the kernel $K_{\phi_{0}}$ is obtained as a Markov kernel on the graph $\left(U, \mathfrak{E}_{U}\right)$ using the pair of weights $(\bar{\mu}, \bar{\pi})$ where

$$
\left\{\begin{array}{l}
\bar{\mu}_{x y}=\beta_{0}^{-1} \phi_{0}(x) \phi_{0}(y) \mu_{x y} \\
\bar{\pi}=\left.\phi_{0}^{2} \pi\right|_{U}
\end{array}\right.
$$

i.e., $K_{\phi_{0}}=\bar{\mu}_{x y} / \bar{\pi}$. Indeed, for any $x, y \in U$, we have

$$
\bar{\mu}_{x y}=\left(\sum_{z \in U} \pi(z)\right) \pi_{\phi_{0}}(x) K_{\phi_{0}}(x, y) \text { and } \bar{\pi}(x)=\left(\sum_{z \in U} \pi(z)\right) \pi_{\phi_{0}}(x) .
$$

Furthermore, $\bar{\mu}$ is subordinated to $\bar{\pi}$ in $U$ because, for any $x \in U$,

$$
\sum_{y \in U} \bar{\mu}_{x y}=\left.\sum_{y \in U} \beta_{0}^{-1} \phi_{0}(x) \phi_{0}(y) \pi\right|_{U}(x) K(x, y)=\left.\phi_{0}(x)^{2} \pi\right|_{U}(x)=\bar{\pi}(x) .
$$

All of this means that we are in precisely the situation of Section 5. We now list four assumptions that will be used to obtain good results concerning the behavior of the chain $\left(K_{\phi_{0}}, \pi_{\phi_{0}}\right)$ by applying the techniques described in Section 5 and Section 6 . In what follows, we always fix the parameter $\alpha \in(0,1]$ as well as $\theta \geq 2$.

For the reader's convenience we give brief pointers to notation that will be used crucially in what follows: John domains (Section 2.1), Whitney coverings (Section 2.2), D-doubling (Definition 3.1, the ball Poincaré inequality (Definition 3.6), elliptic (Definition 3.2), subordinated weight (Definition 3.2), $(\eta, A)$-regular (Definition 5.7), and ( $\eta, A)$-controlled (Definition 5.9). 
Assumption $\mathbf{A} 1$ (on $(\mathfrak{X}, \mathfrak{E}, \pi, \mu)$ ): The measure $\pi$ is $D$-doubling, $\mu$ is adapted and the pair $(\pi, \mu)$ is elliptic and satisfies the $\theta$-Poincaré inequality on balls with constant $P$. In addition, $\mu$ is subordinated to $\pi$.

Assumption A2 (on the finite domain $U$ ): The finite domain $U \subset \mathfrak{X}$ belongs to $J(o, \alpha, R)$ for some $o \in U$ with $R(o, \alpha, U) \leq R \leq 2 R(o, \alpha, U)$.

Assumption A3 (on $U$ in terms of $\phi_{0}$ ): There are $\eta \in(0,1 / 12]$ and $A \geq 1$ such that $\phi_{0}$ is $(\eta, A)$-regular and $A$-doubling on $U$.

Assumption $\mathbf{A 4}$ (on $U$ in terms of $\phi_{0}$ ): There are $\eta \in(0,1 / 12], \omega \geq 0$, and $A \geq 1$ such that $\phi_{0}$ is $(\eta, A)$-regular and $(\omega, A)$-controlled on $U$.

Assumption A1 will be our basic assumption about the underlying weighted graph structure $(\mathfrak{X}, \mathfrak{E}, \pi, \mu)$. Assumption A2 is a strong and relatively sophisticated assumption regarding the geometric properties of the finite domain $U$. Assumptions 3 and 4 are technical requirements necessary to apply the methods in Sections 4 and 5 . In the classical case when the parameter $\theta$ in the assumed Poincaré inequality satisfies $\theta=2$, Assumptions A1-A2 imply that Assumption A4 is satisfied. This follows from Lemma 7.10 below and Lemma 5.12. (Recall that $(\eta, A)$-regular implies $\left(\eta^{\prime}, A\right)$-regular for every $\eta^{\prime}<\eta$. So $(1 / 8, A)$-regular implies $(1 / 12, A)$-regular.)

Lemma 7.10. Assume that A1-A2 are satisfied and $\theta=2$. Then $\phi_{0}$ is $(1 / 8, A)$ regular with $A$ depending only on the quantities $D, P_{e}, P$ appearing in Assumption A1.

Proof: The short outline of the proof is that doubling and Poincare (with $\theta=2$ ) imply the Harnack inequality

$$
\sup _{B}\left\{\phi_{0}\right\} \leq C_{H} \inf _{B}\left\{\phi_{0}\right\}
$$

for any ball $B$ such that $2 B \subset U$. The constant $C_{H}$ is independent of $B$ and $U$ and depends only of $D, P_{e}, P$. This would follow straightforwardly from Delmotte's elliptic Harnack inequality (see Delmotte, 1997) if $\phi_{0}$ were a positive solution of

$$
(I-K) u=0
$$

in the ball $2 B$. However, $\phi_{0}$ is a positive solution of

$$
(I-K) u=\left(1-\beta_{0}\right) u \text {. }
$$

Heuristically, at scale less than $R$, this is almost the same because Assumption A1 implies that $1-\beta_{0} \leq C R^{-2}$. This easy estimate follows by using a tent test function in the ball $B(o, R / 4) \subset U$. To prove the stated Harnack inequality for $\phi_{0}$, one can either extend Delmotte's argument (adapted from Moser's proof of the elliptic Harnack inequality for uniformly elliptic operators in $\mathbb{R}^{n}$ ), see Delmotte (1997), or use the more difficult parabolic Harnack inequality of Delmotte (1999). Indeed, to follow this second approach,

$$
v(t, x)=e^{-\frac{1}{2}\left(1-\beta_{0}\right) t} \phi_{0}(x) \quad\left(\text { resp. } w(t, x)=\left(1-\frac{1}{2}\left(1-\beta_{0}\right)\right)^{t} \phi_{0}(x)\right)
$$

is a positive solution of the continuous-time (resp. discrete-time) parabolic equation

$$
\left[\partial_{t}+\frac{1}{2}(I-K)\right] v=0\left(\operatorname{resp} . \quad w(t+1, x)-w(t, x)=-\left[\frac{1}{2}(I-K) w_{t}\right](x)\right)
$$

in $U$ (in the discrete time case, $w_{t}=w(t, \cdot)$ ). These parabolic equations are associated with the (so-called) lazy version of the Markov kernel $K$, that is, $\frac{1}{2}(I+K)$ to insure that the results of Delmotte (1999) are applicable. The parabolic Harnack 
inequality in Delmotte (1999) necessitates that the time scale be adapted to the size of the ball on which it is applied, namely, the time scale should be $r^{2}$ if the ball has radius $r$. Our positive solution $v(t, x)=e^{-\frac{1}{2}\left(1-\beta_{0}\right) t} \phi_{0}(x)$ of the heat equation is defined on $\mathbb{R} \times B$ where $B=B(z, r) \subset U$ is a ball of radius $r$. The parabolic Harnack inequality gives that there is a constant $C_{H}$ such that, for all $x, y \in B$,

$$
v\left(r^{2}, x\right) \leq C_{H} v\left(2 r^{2}, y\right) .
$$

Because $1-\beta_{0} \leq C R^{-2}$ and $r \leq R$, the exponential factors

$$
e^{-\frac{1}{2}\left(1-\beta_{0}\right) r^{2}}, e^{-\left(1-\beta_{0}\right) r^{2}}
$$

behave like the constant 1 . This implies that $\phi_{0}(x) \approx \phi_{0}(y)$ for all $x, y \in B$.

The following statement is an easy corollary of the last part of the proof of Lemma 7.10. See the remarks following the statement.

Lemma 7.11. Fix $\theta \geq 2$. Assume that $(\mathfrak{X}, \mathfrak{E}, \pi, \mu)$ is such that $\mu$ is adapted, the pair $(\pi, \mu)$ is elliptic and $\mu$ is subordinated to $\pi$. In addition, assume that the operator $\frac{1}{2}\left(I+K_{\mu}\right)$ satisfies the $\theta$-parabolic inequality $\mathrm{PHI}(\theta)$ of Barlow and Bass $(2004,(1.9))$. If $U$ is a finite domain in $\mathfrak{X}$ satisfying $\mathrm{A} 2$, the function $\phi_{0}$ is $(1 / 8, A)$-regular with $A$ depending only on the $\theta, P_{e}$, and the constant $C_{H}$ from the $\theta$-parabolic Harnack inequality.

Remark 7.12. The $\theta$-parabolic inequality $\operatorname{PHI}(\theta)$ of Barlow and Bass (2004) implies the doubling property and the $\theta$-Poincaré inequality (Barlow and Bass, 2004, Theorem 1.5). In addition it implies the so-called cut-off Sobolev inequality $\operatorname{CS}(\theta)$ (Barlow and Bass (2004, Definition 1.4; Theorem 1.5)). Conversely, doubling, the $\theta$ Poincaré inequality and $\operatorname{CS}(\theta)$ imply $\operatorname{PHI}(\theta)$. In the case $\theta=2$, the cut-off Sobolev inequality is always trivially satisfied. When $\theta>2$, the cut-off Sobolev inequality is non-trivial and become essential to the characterization of the parabolic Harnack inequality $\operatorname{PHI}(\theta)$. See Barlow and Bass (2004, Theorem 5) (in Barlow and Bass, 2004 , the parameter $\theta$ is called $\beta$ ).

Remark 7.13. To prove Lemma 7.11, it is essential to have an upper bound $1-\beta_{0} \leq$ $C R^{-\theta}$ on the spectral gap $1-\beta_{0}$. This upper bound easily follows from the cut-off Sobolev inequality $\operatorname{CS}(\theta)$.

We can now state two very general results concerning the reversible Markov chain $\left(K_{\phi_{0}}, \pi_{\phi_{0}}\right)$ in the finite domain $U$. The first theorem has weaker hypotheses and is, in principle, easier to apply. When the parameter $\omega=0$, the two theorems gives essentially identical conclusions. The proofs are immediate application of the results in Section 5 and follow the exact same line of reasoning used in Section 6 to obtain Theorems 6.6-6.7. In the following statement, $\beta_{-}$is the least eigenvalue of the pair $\left(K_{\phi_{0}}, \pi_{\phi_{0}}\right)$ and $\beta$ is second largest eigenvalue of $\left(K_{\phi_{0}}, \pi_{\phi_{0}}\right)$. If $\beta_{U,-}$ denotes the smallest eigenvalue of $K_{U}$ on $L^{2}\left(U, \pi_{U}\right)$, then $\beta_{-}=\beta_{U,-} / \beta_{0}$. If $\beta_{U, 1}$ denotes the second largest eigenvalue of $K_{U}$ on $L^{2}\left(U, \pi_{U}\right)$, then $\beta=\beta_{U, 1} / \beta_{0}$. The eigenfunction $\phi_{0}$ is normalized by $\pi_{U}\left(\phi_{0}^{2}\right)=1$.

Theorem 7.14. Fix $\alpha, \theta, \eta, \omega, P_{e}, P, D, A$ and assume A1-A2-A4. Under these assumptions there are constants $c, C \in(0, \infty)$ (where $c, C$ depend only on the parameters $\alpha, \theta, \eta, \omega, P_{e}, P, D$ and $\left.A\right)$ such that

$$
1-\beta_{0} \leq C R^{-\theta}
$$


and

$$
1-\beta \geq c R^{-(\theta+\omega)} .
$$

Assume further that $1+\beta_{-} \geq c R^{-(\theta+\omega)}$. Then, for all $t \geq R^{\theta+\omega}$, we have the following $L^{\infty}$ rate of convergence,

$$
\max _{x, y \in u}\left|\frac{K_{\phi_{0}}^{t}(x, y)}{\pi_{\phi_{0}}(y)}-1\right| \leq C \exp \left(-c \frac{t}{R^{\theta+\omega}}\right) .
$$

Equivalently, in terms of the kernel $K_{U}$, this reads

$$
\left|K_{U}^{t}(x, y)-\beta_{0}^{t} \phi_{0}(x) \phi_{0}(y) \pi_{U}(y)\right| \leq C \beta_{0}^{t} \phi_{0}(x) \phi_{0}(y) \pi_{U}(y) e^{-c t / R^{\theta+\omega}},
$$

for all $x, y \in U$ and $t \geq R^{\theta+\omega}$.

Remark 7.15. Part of the proof of this result is to show that there are constants $C, \nu$ such that, for all $t \leq R^{\theta+\omega}$ and $x, y \in U$,

$$
\frac{K_{\phi_{0}}^{t}(x, y)}{\pi_{\phi_{0}}(y)} \leq C\left(R^{\theta+\omega} / t\right)^{\nu}
$$

where $C, \nu$ depends only on the parameters $\alpha, \theta, \eta, \omega, P_{e}, P, D$ and $A$. In terms of $K_{U}^{t}$, this becomes for all $t \leq R^{\theta+\omega}$ and $x, y \in U$,

$$
\frac{K_{U}^{t}(x, y)}{\pi_{U}(y)} \leq C\left(R^{\theta+\omega} / t\right)^{\nu} \phi_{0}(x) \phi_{0}(y) .
$$

This type of estimate for $K_{U}^{t}$ is called intrinsic ultracontractivity. It first appeared in the context of Euclidean domains in Davies and Simon (1984); Davies (1985) (see also Davis, 1991) and has been discussed since by many authors. In its classical form, ultracontractivity of the Dirichlet heat semigroup in a bounded Euclidean domain $U$ is the statement that, for each $t>0$, there is a constant $C_{t}$ such that for all $x, y \in U$,

$$
h_{U}^{D}(t, x, y) \leq C_{t} \phi_{0}(x) \phi_{0}(y)
$$

Here $h_{U}^{D}(t, x, y)$ is the fundamental solution (e.g., heat kernel) of the heat equation with Dirichlet boundary condition in $U$. Ultracontractivity may or may not hold in a particular bounded domain. It is known that it holds in bounded Euclidean John domains, see Cipriani (1994). We note here that running the line of reasoning used here in the case of bounded Euclidean John domains would produce more effective ultracontractivity bounds than the ones reported in Cipriani (1994).

Remark 7.16. As mentioned above, Theorem 7.14 is relatively easy to apply. Hypothesis $\mathrm{A} 1$ is our basic working hypothesis regarding $(\mathfrak{X}, \mathfrak{E}, \pi, \mu)$. Hypothesis A2 requires the finite domain $U$ to be a John domain. When $\theta=2$, Hypothesis A4 is automatically satisfied for some $\omega \geq 0$ depending only on the other fixed parameters (Lemma 7.10). When $\theta>2$, we would typically appeal to Lemma 7.11 in order to verify $\mathrm{A} 4$. This requires an additional assumption on $(\mathfrak{X}, \mathfrak{E}, \pi, \mu)$, namely, that $\frac{1}{2}\left(I+K_{\mu}\right)$ satisfies the parabolic Harnack inequality $\operatorname{PHI}(\theta)$ of Barlow and Bass (2004). For instance, Theorem 7.14 applies uniformly to the graph metric balls in $(\mathfrak{X}, \mathfrak{E}, \pi, \mu)$ under Hypothesis $\mathrm{A} 1$ when $\theta=2$, and under $\mathrm{A} 1$ and $\operatorname{PHI}(\theta)$ when $\theta>2$. Consider the infinite Vicsek fractal graph $\left(\mathfrak{X}^{V}, \mathfrak{E}^{V}\right.$ ) (a piece of which is pictured in Figure 3.10) equipped the vertex weight $\pi^{V}(x)=4, x \in \mathfrak{X}^{V}$ and the edge weight $\mu_{x y}^{V}=1,\{x, y\} \in \mathfrak{E}^{V}$. This structure is a good example for the case $\theta>2$. It has $\theta=d+1$ where $d=\log 5 / \log 3$ and also volume growth $\pi(B(x, r)) \asymp r^{d}$. 
It satisfies the parabolic Harnack inequality $\mathrm{PHI}(\theta)$. See, e.g., Barlow et al. (2005, Example 2 and Example 3, Section 5) which provides larger classes of examples of this type.

Theorem 7.17. Fix $\alpha, \theta, \eta, P_{e}, P, D, A$ and assume A1-A2-A3. Under these assumptions there are constants $c, C \in(0, \infty)$ (where $c, C$ depend only on the parameters $\alpha, \theta, \eta, P_{e}, P, D$ and $\left.A\right)$ such that

$$
1-\beta_{0} \leq C R^{-\theta}
$$

and

$$
1-\beta \geq c R^{-\theta} .
$$

Assume further that $1+\beta_{-} \geq c R^{-\theta}$. Then, for all $t \geq R^{\theta}$, we have

$$
\max _{x, y \in u}\left|\frac{K_{\phi_{0}}^{t}(x, y)}{\pi_{\phi_{0}}(y)}-1\right| \leq C \exp \left(-c \frac{t}{R^{\theta}}\right) .
$$

Equivalently, in terms of the kernel $K_{U}$, this reads

$$
\left|K_{U}^{t}(x, y)-\beta_{0}^{t} \phi_{0}(x) \phi_{0}(y) \pi_{U}(y)\right| \leq C \beta_{0}^{t} \phi_{0}(x) \phi_{0}(y) \pi_{U}(y) e^{-c t / R^{\theta}},
$$

for all $x, y \in U$ and $t \geq R^{\theta}$.

Remark 7.18. As for Theorem 7.14, part of the proof of Theorem 7.17 is to show that there are constants $C, \nu$ such that, for all $t \leq R^{\theta}$ and $x, y \in U$,

$$
\frac{K_{\phi_{0}}^{t}(x, y)}{\pi_{\phi_{0}}(y)} \leq C\left(R^{\theta} / t\right)^{\nu}
$$

where $C, \nu$ depends only on the parameters $\alpha, \theta, \eta, P_{e}, P, D$ and $A$. In terms of $K_{U}^{t}$, this gives the intrinsic ultracontractivity estimate for all $t \leq R^{\theta}$ and $x, y \in U$,

$$
\frac{K_{U}^{t}(x, y)}{\pi_{U}(y)} \leq C\left(R^{\theta} / t\right)^{\nu} \phi_{0}(x) \phi_{0}(y)
$$

Remark 7.19. Theorem 7.17 gives a more satisfying result than Theorem 7.14 in that it does not involves the extra parameter $\omega$ (the two theorems have the same conclusion when $\omega=0$ ). However, Theorem 7.17 requires to verify Hypothesis A3, that is, to show that $\pi_{\phi_{0}}$ is doubling. This is an hypothesis that is hard to verify, even for simple finite domains in $\mathbb{Z}^{d}$. At this point in this article, the only finite domains in $\mathbb{Z}^{2}$ for which we could verify this hypothesis are those where we can compute $\phi_{0}$ explicitly such as cubes with sides parallel to the axes or the 45 degree finite cone of Figure 1.1. This shortcoming will be remedied in the next section when we show that finite inner-uniform domains satisfy Hypothesis A3 (see Theorem 8.9).

Example 7.20. We can apply either of these two theorems to the one dimensional example of simple lazy random walk on $\{0,1, \ldots, N\}$ with absorption at 0 and reflection at $N$. This is the leading example of Diaconis and Miclo (2015) where quantitative estimates for absorbing chains are discussed. In this simple example, we know exactly the function $\phi_{0}$ and we can easily verify A1-A2-A3 and A4 with $\omega=0$. In terms of the Doob-transform chain $K_{\phi_{0}}$ and its invariant measure $\pi_{\phi_{0}}$, the result above proves convergence after order $N^{2}$ steps. This improves upon the results of Diaconis and Miclo (2015) by a factor of $\log N$. 
Example 7.21. In the same manner, we can apply the two theorems above to the example discussed in the introduction (Figure 1.1). The key is again the fact that we can find an explicit expression for the eigenfunction $\phi_{0}$ and that it follows that Assumptions A1-A1-A3 and A4 with $\omega=0$ are satisfied. The conclusion is the same. In terms of the Doob-transform chain $K_{\phi_{0}}$ and its invariant measure $\pi_{\phi_{0}}$, the result above proves convergence after order $N^{2}$ steps.

Example 7.22. Let us focus on the square grid $\mathbb{Z}^{m}$ in a fixed dimension $m$ and on the family of its finite $\alpha$-John domains for some fixed $\alpha \in(0,1]$. In addition, for simplicity, we assume that the weight $\mu$ is constant equal to $1 /(4 m)$ on the grid edges and $\pi \equiv 1$ (this insure aperiodicity of $K$ and $K_{U}$ ). Obviously, A1 is satisfied with $\theta=2$ and A2 is assumed since $U$ is an $\alpha$-John domain. Theorem 7.17 does not apply here because we are not able to prove doubling of the measure $\pi_{\phi_{0}}$ (and in fact, doubling should probably not be expected in this generality). However, there is an $\omega$ (which depends only on the two fixed parameters $m$ and $\alpha$ ) such that A4 is satisfied (this follows from Lemma 5.12 and Lemma 7.10), and hence, we can apply Theorem 7.14.

Theorem 7.23. Fix $m$ and $\alpha \in(0,1]$. Let the square grid $\mathbb{Z}^{m}$ be equipped with the weights $\mu, \pi$ described above. There are constants $c=c(m, \alpha), C=C(m, \alpha)$ and $\omega=\omega(m, \alpha)$ such that, for any finite $\alpha$-John domain $U$ in $\mathbb{Z}^{m}$ with John radius $R_{U}=R(o, \alpha, U)$, the Doob-transform chain $K_{\phi_{0}}$ satisfies

and, for $t \geq R^{2+\omega}$

$$
\begin{gathered}
c R_{U}^{-2} \leq 1-\beta_{0} \leq C R_{U}^{-2}, \\
1-\beta \geq c R_{U}^{-2-\omega},
\end{gathered}
$$

$$
\max _{x, y \in u}\left|\frac{K_{\phi_{0}}^{t}(x, y)}{\pi_{\phi_{0}}(y)}-1\right| \leq C \exp \left(-c \frac{t}{R_{U}^{2+\omega}}\right) .
$$

Equivalently, in terms of the kernel $K_{U}$, this reads

$$
\left|K_{U}^{t}(x, y)-\beta_{0}^{t} \phi_{0}(x) \phi_{0}(y) \pi_{U}(y)\right| \leq C \beta_{0}^{t} \phi_{0}(x) \phi_{0}(y) \pi_{U}(y) e^{-c t / R^{2+\omega}},
$$

for all $x, y \in U$ and $t \geq R^{2+\omega}$. Moreover, for $1 \leq t \leq R^{\theta+\omega}$, we have

$$
\frac{K_{U}^{t}(x, y)}{\pi_{U}(y)} \leq C\left(R^{2+\omega} / t\right) \phi_{0}(x) \phi_{0}(y)
$$

It is an open question whether or not it is possible to prove the above theorem with $\omega(m, \alpha)=0$ for all finite $\alpha$-John domains in $\mathbb{Z}^{m}$ or, even more generally, for a general underlying structure $(\mathfrak{X}, \mathfrak{E}, \pi, \mu)$ under assumption $A 1$ with $\theta=2$.

Remark 7.24. Recall from Definition 7.7 that $\nu_{x}^{t}(y)=\mathbf{P}_{x}\left(X_{t}=y \mid \tau_{u}>t\right)$. Theorem 7.9 gives control on the rate of convergence of $\nu_{x}^{t}(y)$ in terms of the rate of convergence of $K_{\phi_{0}}^{t}(x, y)$. We can now apply Theorem 7.9 in each of the settings described above in Theorems 7.14, 7.17, and 7.23. For example, in the case of the square grid $\mathbb{Z}^{m}$ and for a fixed $\alpha \in(0,1)$, there exists $\omega=\omega(m, \alpha) \geq 0$ and $C=(m, \alpha), c=c(m, \alpha)>0$ such that, for any finite $\alpha$-John domain $U$ with John radius $R$,

$$
\forall t>C R^{2+\omega},\left|\frac{\phi_{0}(y) \nu_{x}^{t}(y)}{\sum_{z \in U} \phi_{0}(z)}-1\right| \leq e^{-c t / R^{2+\omega}}
$$




\section{Inner-uniform domains}

We now turn to the definition of inner $(\alpha, A)$-uniform domains. These domains form a subclass of the class of $\alpha$-John domains. They allow for a much more precise analysis of Metropolis-type chains and their Doob-transforms.

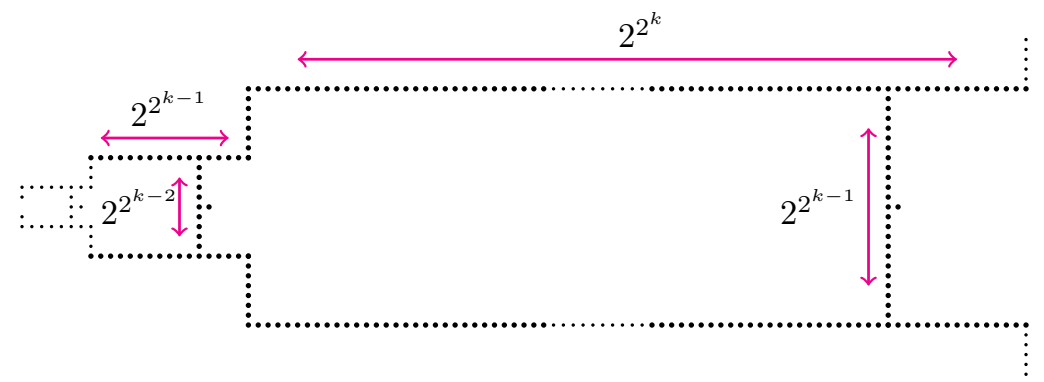

FiguRE 8.13. A graph in which, at large scales, some balls are not inner-uniform. To the left, the graph ends after finitely many step with an origin $o$ which serves as the center of the balls to be considered. To the right, the indicated pattern is repeated infinitely many times at larger and larger scales. This graph is roughly linear. It satisfies doubling and Poincaré.

Although the definition of inner-uniform domains given below appears to be quite similar to that of John domains, it is in fact much harder, in general, to find inneruniform domains than it is to find John domains. In the square lattice $\mathbb{Z}^{d}$, both classes of domains are very large and contain many interesting natural examples. Things are very different if one consider an abstract graph structure $(\mathfrak{X}, \mathfrak{E})$ of the type used in this paper. We noted earlier that any graph distance ball $B(o, r)$ in such a structure $(\mathfrak{X}, \mathfrak{E})$ is a 1-John domain. In particular, $\mathfrak{X}$ admits an exhaustion $\mathfrak{X}=\lim _{r \rightarrow \infty} B(o, r)$ by finite 1-John domains. We know of no constructions of an increasing family of $\alpha$-inner-uniform domains in $(\mathfrak{X}, \mathfrak{E})$, in general. Even if we assume additional properties such as doubling and Poincaré inequality on balls, we are not aware of a general method to construct inner-uniform subsets. Of course, it may happen that, as in the case of $\mathbb{Z}^{d}$, graph balls turn out to be inner-uniform (all for some fixed $\alpha>0$ ). But that is not the case in general. Figures 8.13 and 8.14 describe a simple planar graph in which, there are balls $B\left(o, r_{i}\right)$ with $r_{i}$ tending to infinity which each contains points $x_{i}, y_{i}$ such that $d_{B\left(o, r_{i}\right)}\left(x_{i}, y_{i}\right)=\rho_{i}=o\left(r_{i}\right)$ but the only path from $x_{i}$ to $y_{i}$ of length $O\left(\rho_{i}\right)$ has a middle point $z_{i}$ which is at distance 1 from the boundary. All other paths from $x_{i}$ to $y_{i}$ have length at least $r_{i} / 8$. This implies that the inner-uniformity constant $\alpha_{i}$ of the ball $B\left(o, r_{i}\right)$ is $O\left(\rho_{i} / r_{i}\right) \leq o(1)$. The graph in question has a very simple structure and it satisfies doubling and the Poincaré inequality on balls at all scales.

\subsection{Definition and main convergence results.}

Definition 8.1. A domain $U$ in $\mathfrak{X}$ is an inner $(\alpha, A)$-uniform domain (with respect to the graph structure $(\mathfrak{X}, \mathfrak{E}))$ if for any two points $x, y \in U$ there exists a path $\gamma_{x y}=\left(x_{0}=x, x_{1}, \ldots, x_{k}=y\right)$ joining $x$ to $y$ in $\left(U, \mathfrak{E}_{U}\right)$ with the properties that: 


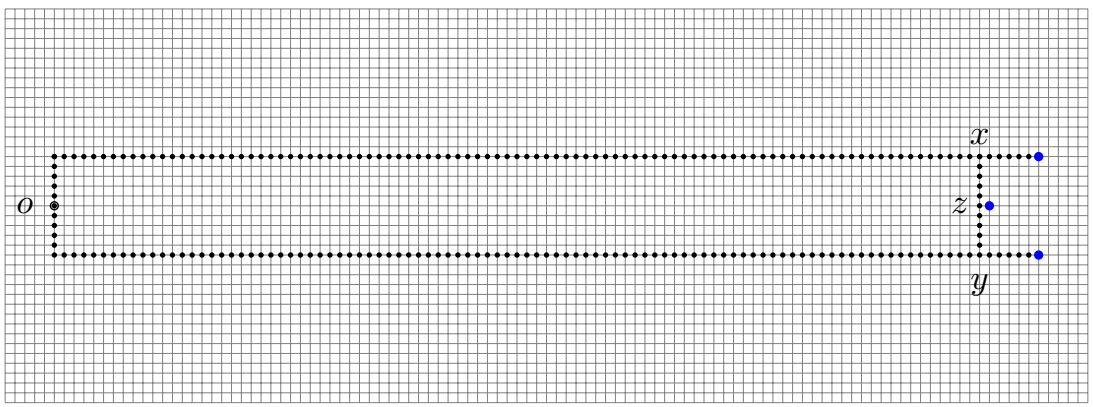

Figure 8.14. The basic model for the balls in the graph of Figure 8.13. The shortest path from $x$ to $y$ is much shorter than any other path but its middle point is at distance 1 from the boundary of the ball marked by blue dots.

(1) $k \leq A d_{U}(x, y)$;

(2) For any $j \in\{0, \ldots, k\}, d\left(x_{j}, \mathfrak{X} \backslash U\right) \geq \alpha(1+\min \{j, k-j\})$.

Remark 8.2. Because the second condition must hold for all $x$, including those that are distance 1 from the boundary, we see that $\alpha \in(0,1]$.

We can think of an inner-uniform domain $U$ as being one where any two points are connected by a banana-shaped region. The entire banana must be contained within $U$. See Figure 8.15 for an illustration.

There is an alternative and equivalent (modulo a change in $\alpha$ ) definition of inner-uniformity which uses distance instead of path-length in the second condition. More precisely, in this alternative definition, the condition "for any $j=0, \ldots, k$, $d\left(x_{j}, \mathfrak{X} \backslash U\right) \geq \alpha(1+\min \{j, k-j\})$ " is replaced by "for any $j=0, \ldots, k, d\left(x_{j}, \mathfrak{X} \backslash U\right) \geq$ $\alpha^{\prime} \min \left\{d_{U}\left(x_{j}, x\right), d_{U}\left(x_{j}, y\right)\right\}$ ". It is obvious that the definition we choose here easily implies the condition of the alternative definition (with $\alpha=\alpha^{\prime}$ ). The reverse implication is much less obvious. It amounts to showing that it is possible to choose the path $\gamma_{x y}$ so that any of its segments $\left(x_{i}, x_{i+1}, \ldots, x_{j}\right)$ provide approximate geodesics between its end-points. This requires a modification (i.e., straightening) of the path $\gamma_{x y}$ provided by the definition because there is no reason these paths have this property. See Martio and Sarvas (1979).

The following lemma shows that all inner-uniform domains are John domains. However, the converse is not true. See Figure 8.16.

Lemma 8.3. Suppose that $U$ is a finite inner $(\alpha, A)$-uniform domain. Let o be a point such that $d(o, \mathfrak{X} \backslash U)=\max \{d(x, \mathfrak{X} \backslash U): x \in U\}$, and let $R=d(o, \mathfrak{X} \backslash U)$. Then $U \in J\left(\mathfrak{X}, \mathfrak{E}, o, \alpha^{2} / 8,(2 / \alpha) R\right)$, that is, $U$ is an ( $\left.\alpha^{2} / 8\right)$-John domain.

Proof: Look at the mid-point $z=x_{\lfloor k / 2\rfloor}$ along $\gamma_{x o}$. We have $R \geq d(z, \mathfrak{X} \backslash U)$ $\geq \alpha k / 2$ so the $k \leq(2 / \alpha) R$. We consider three cases to find a lower bound on $d\left(x_{j}, \mathfrak{X} \backslash U\right)$ along $\gamma_{x o}$.

(1) When $j \leq k / 2$, then we have $d\left(x_{j}, \mathfrak{X} \backslash U\right) \geq \alpha(1+j)$.

(2) When $x_{j} \in B(o, R / 2)$, then we have that

$$
d\left(x_{j}, \mathfrak{X} \backslash U\right) \geq R / 2 \geq(\alpha / 4) k \geq(\alpha / 8)(1+j) .
$$



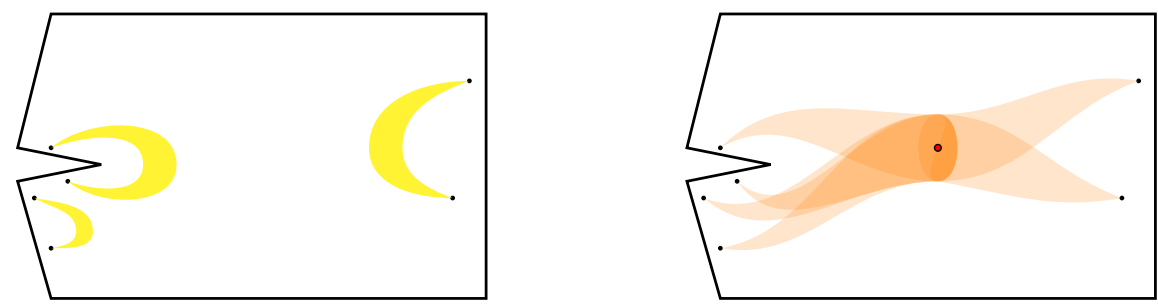

FiguRe 8.15. On the left: The banana regions for arbitrary pairs of points which are the witnesses for the inner-uniform property. On the right: The carrot regions joining arbitrary vertices to the central point $o$ marked in red. They are witnesses for the John domain property.

(3) When $x_{j} \notin B(o, R / 2)$ and $j \geq k / 2$, then $k-j \geq R / 2$ and

$$
d\left(x_{j}, \mathfrak{X} \backslash U\right) \geq \alpha(1+k-j) \geq \alpha(1+R / 2) \geq \alpha(1+\alpha k / 4) \geq\left(\alpha^{2} / 4\right)(1+j) \text {. }
$$

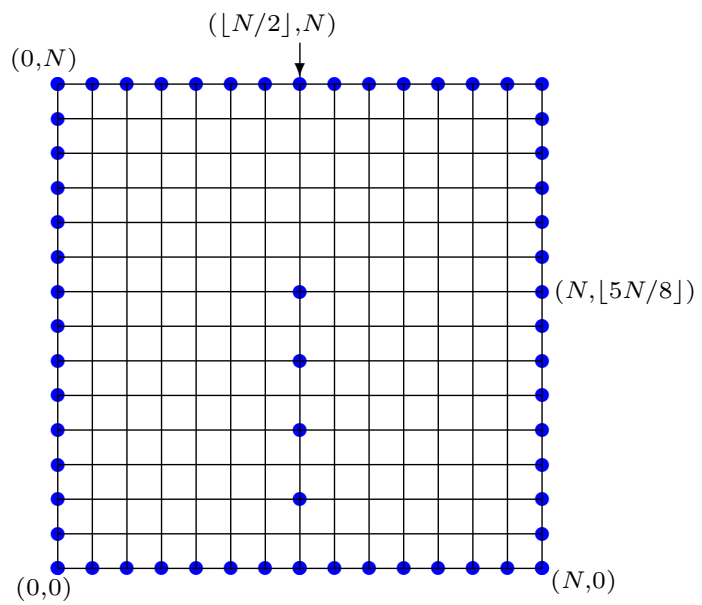

Figure 8.16. A domain that is John but not inner-uniform. The blue dots are the boundary. Note that, on the middle vertical line, the blue dots are placed on every other vertex, up to the indicated height.

Remark 8.4. The word "inner" in inner-uniform refers to the fact that the first condition compares the length $k$ of the curve $\gamma_{x y}$ to the inner-distance $d_{U}(x, y)$ between $x$ and $y$. If, instead, the original distance $d(x, y)$ is used (i.e., the first condition in the definition becomes $k \leq A d(x, y))$, then we obtain a much more restrictive class of domains called "uniform domains." See Figure 8.18. 


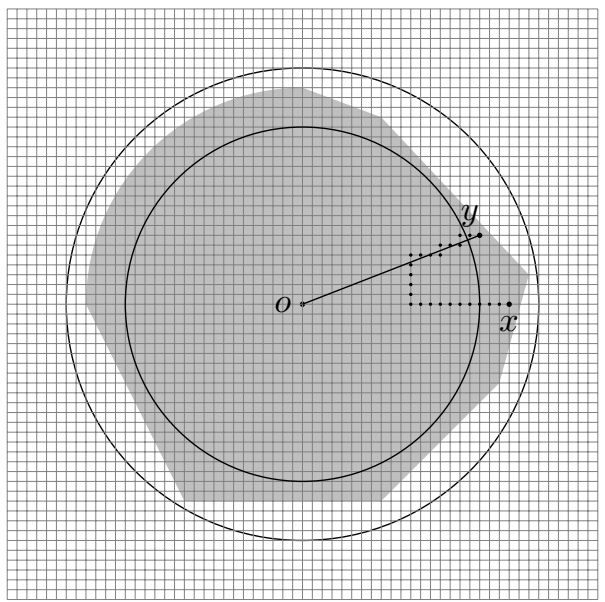

FiguRE 8.17. Finite discrete "convex subsets" of $\mathbb{Z}^{2}$ are (inner-)uniform

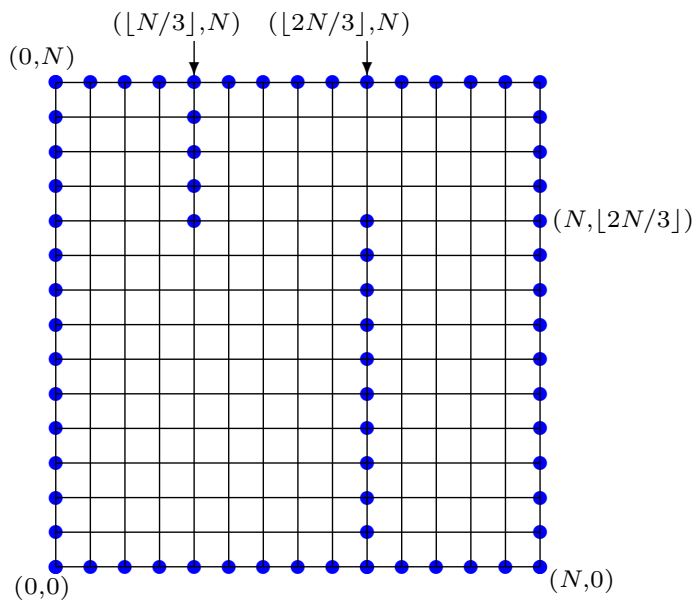

FiguRE 8.18. A domain that is inner-uniform but not uniform.

Example 8.5. The set $\mathfrak{X}_{N}$ in Figure 1.1 (a forty-five degree finite cone in $\mathbb{Z}^{2}$ ) is a uniform domain, and hence, also an inner-uniform domain. Finite convex sets in $\mathbb{Z}^{d}$ in the sense of Example 2.11 are uniform domains, all with the same fixed $(\alpha, A)$ depending only on the dimension $d$. The domain pictured in Figure 2.6 is a uniform domain, with the same fixed $(\alpha, A)$ for all $N$. Note that in this example, viewed as a subset of $\mathbb{Z}^{2}$, some of the boundary points are not killing points, but points where the process is reflected. This illustrates how variations of this type (i.e., with reflecting points) can be treated with our methods.

Example 8.6. In Example 2.10, we observed that metric balls are always 1-John domains. They are not always inner-uniform domains. See Figures 8.13 and 8.14. 
Example 8.7. The discrete "finite convex subsets" $U$ of $\mathbb{Z}^{d}$ satisfying (A.1) and considered in Proposition A.1 are inner-uniform with parameter $\bar{\alpha}>0$ depending only on the dimension $d$ and the parameter $\alpha$ in (A.1). Note that the inner distance in such a finite connected set is comparable to the graph distance of $\mathbb{Z}^{d}$ with comparison constant depending only on the dimension $d$ and the parameter $\alpha$ in (A.1) (i.e., these finite domains are uniform).

Here is a rough description of the paths $\gamma_{x y}$ that demonstrate that such domains $U$ are inner-uniform. (See Figure 8.17). Let $r$ be the distance between $x$ and $y$ in $\mathbb{Z}^{d}$. Recall that $U$ has "center" $o$ and that we can go from $x$ (and $y$ ) to $o$ while getting away linearly from the boundary, roughly along a straight-line (see Proposition A.1). Let $\tilde{x}$ and $\tilde{y}$ be respective points along the paths joining $x$ and $y$ to $o$, respectively at distance $r$ from $x$ and from $y$. Convexity insures that there is a discrete path in $U$ joining $\tilde{x}$ to $\tilde{y}$ while staying close to the straight-line segment between these two points. This discrete path from $\tilde{x}$ to $\tilde{y}$ has length at most $A r$ and stays at distance at least ar from the boundary. This completes the discussion of the example.

Now we return to the general setting. We define a special point $x_{r}$ for each point $x \in U$ and radius $r>0$. The meaning of this definition and the key geometric property of $x_{r}$ is that $x_{r}$ is a point which is essentially as far away from the boundary as possible while still being within a ball of radius $r$ of $x$, i.e., $d\left(x, x_{r}\right) \leq r$. Namely, $d\left(x_{r}, \mathfrak{X} \backslash U\right) \geq \alpha(1+r)$ if $r \leq R$ and $x_{r}=o$ otherwise.

Definition 8.8. Let $U$ be a finite inner $(\alpha, A)$-uniform domain. Let $o$ be a point such that $d(o, \mathfrak{X} \backslash U)=\max \{d(x, \mathfrak{X} \backslash U): x \in U\}=R$. Let $\gamma_{x y}$ be a collection of inner $(\alpha, A)$-uniform paths from $x \in U$ to $y \in U$. For any $x \in U$ and $r>0$, let $x_{r}$ be defined by

$$
x_{r}=\left\{\begin{array}{cl}
x_{\lfloor r\rfloor} & \text { if } \gamma_{x o}=\left(x=x_{0}, x_{1}, \ldots, x_{k}=o\right) \text { with } k \geq r, \\
o & \text { if } \gamma_{x o}=\left(x=x_{0}, x_{1}, \ldots, x_{k}=o\right) \text { with } k<r .
\end{array}\right.
$$

The following Carleson-type theorem, regarding the eigenfunction $\phi_{0}$, is the key to obtaining refined results for the convergence of the intrinsic Doob-transform chain on a finite inner-uniform domain. The context is as follows. In addition to the geometric structure $(\mathfrak{X}, \mathfrak{E})$, we assume we are given a measure $\pi$ and an edge weight $\mu$ such that $(\mathfrak{X}, \mathfrak{E}, \pi, \mu)$ satisfies Assumption A1 with $\theta=2$, i.e., we assume that the measure $\pi$ is $D$-doubling, $\mu$ is adapted, $\pi$ dominates $\mu$, and the pair $(\pi, \mu)$ is elliptic and satisfies the 2-Poincaré inequality on balls with constant $P$.

Theorem 8.9. Assume $(\mathfrak{X}, \mathfrak{E}, \pi, \mu)$ satisfies Assumption $\mathrm{A} 1$ with $\theta=2$ and fix $\alpha, A$. There exists a constant $C_{0}$ depending only on $\alpha, A, D, P_{e}, P$ such that, for any finite inner $(\alpha, A)$-uniform domain $U$, the positive eigenfunction $\phi_{0}$ for the kernel $K_{U}$ in $U$ is $\left(1 / 8, C_{0}\right)$-regular and satisfies

$$
\forall r>0, x \in U, z \in B_{U}(x, r / 2), \phi_{0}(z) \leq C_{0} \phi_{0}\left(x_{r}\right) .
$$

Corollary 8.10. Under the assumptions of Theorem 8.9, there are constants $D_{0}$, $D_{1}$ depending only on $\alpha, A, D, P_{e}, P$ such that

$$
\forall x \in U, r>0, \pi_{\phi_{0}}\left(B_{U}(x, 2 r)\right) \leq D_{0} \pi_{\phi_{0}}\left(B_{U}(x, r)\right) .
$$

Moreover, for all $r \in[0, R]$,

$$
D_{1}^{-1} \phi_{0}\left(x_{r}\right)^{2} \pi_{U}\left(B\left(x_{r}, \alpha r\right)\right) \leq \pi_{\phi_{0}}\left(B_{U}(x, r)\right) \leq D_{1} \phi_{0}\left(x_{r}\right)^{2} \pi_{U}\left(B\left(x_{r}, \alpha r\right)\right) .
$$


The following corollary gives a rate of convergence of the Doob transform chain to its stationary distribution in $L^{\infty}$.

Corollary 8.11. Under the assumptions of Theorem 8.9, there are constants $C, c$ depending only on $\alpha, A, D, P_{e}, P$ such that, assuming that the lowest eigenvalue $\beta_{-}$ of the reversible Markov chain $\left(K_{\phi_{0}}, \pi_{\phi_{0}}\right)$ satisfies $1+\beta_{-} \geq c R^{-2}$, we have

$$
\max _{x, y \in U}\left|\frac{K_{\phi_{0}}^{t}(x, y)}{\pi_{\phi_{0}}(y)}-1\right| \leq C \exp \left(-c \frac{t}{R^{2}}\right),
$$

for all $t \geq R^{2}$. In terms of the kernel $K_{U}$, this reads

$$
\left|K_{U}^{t}(x, y)-\beta_{0}^{t} \phi_{0}(x) \phi_{0}(y) \pi_{U}(y)\right| \leq C \beta_{0}^{t} \phi_{0}(x) \phi_{0}(y) \pi_{U}(y) e^{-c t / R^{2}},
$$

for all $x, y \in U$ and $t \geq R^{2}$.

Proof: This follows from Theorem 7.17 because the measure $\phi_{0}^{2} \pi_{U}$ is doubling by Theorem 8.9 (and $U$ is a John domain by Lemma 8.3).

Corollary 8.12. Under the assumptions of Theorem 8.9, there are constants $c, C$ depending only on $\alpha, A, D, P_{e}, P$ such that the second largest eigenvalue $\beta$ of the reversible Markov chain $\left(K_{\phi_{0}}, \phi_{\phi_{0}}\right)$ satisfies

$$
c R^{-2} \leq 1-\beta \leq C R^{-2} .
$$

If $\beta_{U, 1}<\beta_{U, 0}=\beta_{0}$ denotes the second largest eigenvalue of the kernel $K_{U}$ acting on on $L^{2}\left(U, \pi_{U}\right)$ then $\beta=\beta_{U, 1} / \beta_{0}$ and

$$
c R^{-2} \beta_{0} \leq \beta_{0}-\beta_{U, 1} \leq C \beta_{0} R^{-2}
$$

or equivalently

$$
\beta_{0}\left(1-C R^{-2}\right) \leq \beta_{U, 1} \leq \beta_{0}\left(1-c R^{-2}\right) .
$$

In particular, for all $t$,

$$
\max _{x \in U} \sum_{y \in U}\left|K_{\phi_{0}}^{t}(x, y)-\pi_{\pi_{0}}(y)\right| \geq c e^{-C t / R^{2}} .
$$

The following theorem is closely related to Theorem 8.9 and is to used to obtain explicit control on the function $\phi_{0}$. In Section 9 we demonstrate the power of this theorem in several examples.

Theorem 8.13. Assume A1 with $\theta=2$ and fix $\alpha, A$. There exists a constant $C_{1}$ depending only on $\alpha, A, D, P_{e}, P$ such that, for any finite inner $(\alpha, A)$-uniform domain $U$, any point $x \in U$ and $r>0$ such that

$$
B_{U}(x, r)=\left\{y \in U: d_{U}(x, y) \leq r\right\} \neq U
$$

and any function $h$ defined in $U$ and satisfying $K_{U} h=h$ in $B_{U}(x, r)$, we have

$$
\forall y, z \in B_{U}(x, r / 2), \quad \frac{\phi_{0}(y)}{\phi_{0}(z)} \leq C_{1} \frac{h(y)}{h(z)} .
$$


8.2. Proofs of Theorems 8.9 and 8.13: the cable space with loops. The statement in Theorem 8.9 is a version of a fundamental inequality known as a Carleson estimate Carleson (1962) and was first derived in the study of analysis on Lipschitz domains Kemper (1972); Ancona (1978); Dahlberg (1977); Wu (1978). For a modern perspective, sharp results, and references to the vast literature on the subject in the context of analysis on bounded domains, see Bañuelos et al. (1991); Aikawa (2001, 2005, 2008, 2015). The generality and flexibility of the arguments developed by H. Aikawa in these papers and other works, based on the notion of "capacity width," is used in a fundamental way in Gyrya and Saloff-Coste (2011) and in Lierl and Saloff-Coste $(2014 b, a)$; Lierl $(2015,2018)$ to extend the result in the setting of (nice) Dirichlet spaces.

Given $(\mathfrak{X}, \mathfrak{E}, \mu, \pi)$ one can build an associated continuous space $\mathbf{X}$, known as the cable space for $(\mathfrak{X}, \mathfrak{E}, \pi, \mu)$. In many cases, it is more difficult to prove theorems in discrete domains than in continuous domains - the cable space provides an important bridge by allowing us to transfer known theorems from the continuous space $\mathbf{X}$ to its associated discrete space $\mathfrak{X}$.

Topologically, the space $\mathbf{X}$ is a connected one-dimensional complex, that is, a union of copies of the interval $[0,1]$ with some identifications of end points. The process of building the cable space from the discrete space $(\mathfrak{X}, \mathfrak{E}, \mu, \pi)$ is straightforward: the zero-dimensional points in the complex are given by the vertices $\mathfrak{X}$; two points $x$ and $y$ are then connected by a unit length edge $[0,1]$ if $\{x, y\} \in \mathbb{E}$, with 0 identified with $x$ and 1 identified with $y$. For early references to the cable space, see the introduction to Cattaneo (1997). This resource is particularly relevant because it discusses the spectrum of the discrete Laplacian.

But we need to allow for the addition of self-loops, copies of $[0,1]$ with 0 and 1 identified to each other and to some vertex $x \in \mathfrak{X}$. (Recall that $\mathfrak{E}$ has no self-loops.) We will use the notation $(0,1)_{x x}$ for the self-loop at $x$ minus the point $x$ itself. Let $\mathfrak{L}$ be the subset of those $x \in \mathfrak{X}$ where $\sum_{y \in U} \mu_{x y}<\pi(x)$. Form a loop at each $x \in \mathfrak{L}$ and set the weight $\mu_{x x}$ on the loop to be equal to its "deficiency,"

$$
\mu_{x x}=\pi(x)-\sum_{y \in U} \mu_{x y}, \quad x \in \mathfrak{L} .
$$

In what follows we will use the notation $x y$ as an index running over $\{x, y\} \in \mathfrak{E}$ when $x \neq y$ and $x \in \mathfrak{L}$ when $x=y$.

We need to use a simple (but rather interesting) variation on this construction. We introduce a loop-parameter, call it $\ell$. For any fixed $\ell \in[0,1]$, we construct the cable space $\mathbf{X}_{\ell}$ as described above but the self-loops have length $\ell$ instead of 1 above. The other edges (non-self-loops) still have length 1.

More precisely, the space $\mathbf{X}_{\ell}$ is obtained by joining any two points $x, y$ in $\mathfrak{X}$ with $\{x, y\} \in \mathfrak{E}$ by a continuous edge $e_{x y}=(0,1)_{x y}$ isometric to the interval $(0,1)$ and adding a self-loop $e_{x x}=(0, \ell)_{x x}$ at each $x \in \mathfrak{L}$. Strictly speaking,

$$
\mathbf{X}=\mathfrak{X} \cup\left(\bigcup_{\{x, y\} \in \mathfrak{E}}(0,1)_{x y}\right) \cup\left(\bigcup_{x \in \mathfrak{L}}(0, \ell)_{x x}\right)
$$

with $e_{x y}$ being a copy of $(0,1)$ when $x \neq y$ and a copy of $(0, \ell)$ when $x=y$. See Figure 8.19. The topology of this space is generated by the open subintervals of these many copies of $(0,1)$ and $(0, \ell)$, together with the star-shaped open neighborhoods of the vertices in $\mathfrak{X}$. 


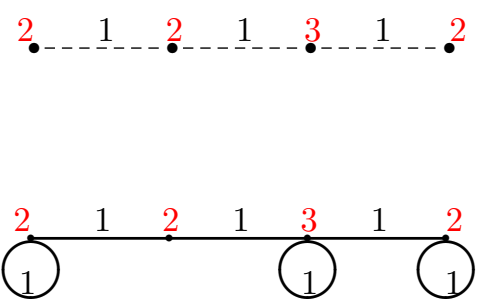

Figure 8.19. A simple example of $(\mathfrak{X}, \mathfrak{E}, \pi, \mu)$ and the associated cable spaces $\mathbf{X}_{\ell}$, where the edge weights $\mu$ are indicated in black and the vertex weights $\pi$ are indicated in red. The black weights on the loops indicate the "deficiencies" in the edge weights, as described in (8.1).

The cable Dirichlet space associated with the data $(\mathfrak{X}, \mathfrak{E}, \mu, \pi, \ell)$ is obtained by equipping $\mathbf{X}_{\ell}$ with its natural distance function $\mathbf{d}_{\ell}: \mathbf{X}_{\ell} \times \mathbf{X}_{\ell} \rightarrow[0, \infty)$, the length of the shortest path between two points. The space $\mathbf{X}_{\ell}$ is also equipped with a measure $\boldsymbol{\pi}$ equal to $\mu_{x y} d t$ on each interval $e_{x y}$ (including the intervals $e_{x x}$ ), and with the Dirichlet form obtained by closing the form

$$
\mathcal{E}_{\mathbf{X}_{\ell}}(f, f)=\sum_{x, y \in \mathfrak{X}} \mu_{x y} \int_{e_{x y}}\left|f_{e_{x y}}^{\prime}(t)\right|^{2} d t, \quad f \in \mathcal{D}_{0}\left(\mathbf{X}_{\ell}\right),
$$

where $\mathcal{D}_{0}\left(\mathbf{X}_{\ell}\right)$ is the space of all compactly supported continuous functions on $\mathbf{X}_{\ell}$ which have a bounded continuous derivative $f_{e_{x y}}^{\prime}$ on each open edge $e_{x y}$ and $e_{x x}$. (Note that the values of these various edge-derivatives at a vertex do not have to match in any sense.) The domain of $\mathcal{E}_{\mathbf{X}_{\ell}}, \mathcal{D}\left(\mathcal{E}_{\mathbf{X}_{\ell}}\right)$, is the closure of $\mathcal{D}_{0}\left(\mathbf{X}_{\ell}\right)$ under the norm

$$
\|f\|_{\mathcal{E}_{\mathbf{X}_{\ell}}}=\left(\int_{\mathbf{X}_{\ell}}|f|^{2} d \boldsymbol{\pi}+\mathcal{E}_{\mathbf{X}_{\ell}}(f, f)\right)^{1 / 2} .
$$

For two function $f$ and $g$, we can define $\mathcal{E}_{\mathbf{X}_{\ell}}(f, g)$ by the polarization formula:

$$
\mathcal{E}_{\mathbf{X}_{\ell}}(f, g)=\frac{1}{4}\left[\mathcal{E}_{\mathbf{X}_{\ell}}(f+g, f+g)-\mathcal{E}_{\mathbf{X}_{\ell}}(f-g, f-g)\right]
$$

The cable Dirichlet space $\left(\mathbf{X}_{\ell}, \boldsymbol{\pi}, \mathcal{E}_{\mathbf{X}_{\ell}}\right)$ is a regular strictly local Dirichlet space (see, e.g., Fukushima et al., 2011; Gyrya and Saloff-Coste, 2011) and its intrinsic distance is the shortest-path distance $\mathbf{d}_{\ell}$ described briefly above. This Dirichlet space is actually quite elementary in the sense that it is possible to describe concretely the domain of the associated Laplacian, the generator of the associated Markov semigroup of operators acting on $L^{2}\left(\mathbf{X}_{\ell}, \boldsymbol{\pi}\right)$. First, we recall that this Laplacian is the self-adjoint operator $\Delta_{\ell}$ with domain $\mathcal{D}\left(\Delta_{\ell}\right)$ in $L^{2}\left(\mathbf{X}_{\ell}, \boldsymbol{\pi}\right)$ defined by

$$
\mathcal{D}\left(\Delta_{\ell}\right)=\left\{u \in \mathcal{D}\left(\mathcal{E}_{\mathbf{X}_{\ell}}\right): \exists C \text { such that, } \forall f \in \mathcal{D}_{0}\left(\mathbf{X}_{\ell}\right), \mathcal{E}_{\mathbf{X}_{\ell}}(u, f) \leq C\|u\|_{2}\right\}
$$


For any function $u \in \mathcal{D}\left(\Delta_{\ell}\right)$ there exists a unique function $v \in L^{2}\left(\mathbf{X}_{\ell}, \boldsymbol{\pi}\right)$ such that $\mathcal{E}_{\mathbf{X}_{\ell}}(u, f)=\int_{\mathbf{X}_{\ell}} v f d \boldsymbol{\pi}$ (from the Riesz representation theorem) and we set

$$
\Delta_{\ell} u=-v .
$$

This implies that

$$
\mathcal{E}_{\mathbf{X}_{\ell}}(u, f)=-\int f \Delta_{\ell} u d \boldsymbol{\pi}
$$

for all $u \in \mathcal{D}\left(\Delta_{\ell}\right)$ and all $f \in \mathcal{D}_{0}\left(\mathbf{X}_{\ell}\right)$ (equivalently, all $f \in \mathcal{D}\left(\mathcal{E}_{\mathbf{X}_{\ell}}\right)$ ).

From the above abstract definition, we can now derive a concrete description of $\mathcal{D}\left(\Delta_{\ell}\right)$. We start with a concrete description of $\mathcal{D}\left(\mathcal{E}_{\mathbf{X}_{\ell}}\right)$. A function $f$ is in $\mathcal{D}\left(\mathcal{E}_{\mathbf{X}_{\ell}}\right)$ if it is continuous on $\mathbf{X}_{\ell}$, belongs to $L^{2}\left(\mathbf{X}_{\ell}, \boldsymbol{\pi}\right)$ and the restriction $f_{e_{x y}}$ of $f$ to any open edge $(0,1)_{x y}$, has a distributional derivative which can be represented by a square-integrable function $f_{e_{x y}}^{\prime}$ satisfying

$$
\sum_{x, y \in \mathfrak{X}} \mu_{x y} \int_{e_{x y}}\left|f_{e_{x y}}^{\prime}\right|^{2} d t<\infty .
$$

The key observation is that, because of the one-dimensional nature of $\mathbf{X}$, on any edge $e_{x y}$ (or subinterval of $e_{x y}$ ) on which $f^{\prime}$ is defined in the sense of distributions and represented by a square integrable function, we have

$$
\left|f\left(s_{2}\right)-f\left(s_{1}\right)\right| \leq \sqrt{\left|s_{2}-s_{1}\right|}\left(\int_{s_{1}}^{s_{2}}\left|f^{\prime}(s)\right|^{2} d s\right)^{1 / 2} .
$$

We now give a (well-known) concrete description of $\mathcal{D}\left(\Delta_{\ell}\right)$. A function $u \in$ $L^{2}\left(\mathbf{X}_{\ell}, \boldsymbol{\pi}\right)$ is in $\mathcal{D}\left(\Delta_{\ell}\right)$ if and only if

(1) The function $u \in L^{2}\left(\mathbf{X}_{\ell}, \boldsymbol{\pi}\right)$ admits a continuous version, which, abusing notation, we still call $u$.

(2) On each open edge $e_{x y}$, the restriction $u_{e_{x y}}$ of $u$ to $e_{x y}$ has a continuous first derivative $u_{e_{x y}}^{\prime}$ with limits at the two end-points and such that

$$
\sum_{x, y \in \mathfrak{X}} \mu_{x y} \int_{e_{x y}}\left|u_{e_{x y}}^{\prime}\right|^{2} d t<\infty .
$$

Furthermore $u_{e_{x y}}$ has a second derivative in the sense of distributions which can be represented by a square-integrable function $u_{e_{x y}}^{\prime \prime}$ and

$$
\sum_{x, y \in \mathfrak{X}} \mu_{x y} \int_{e_{x y}}\left|u_{e_{x y}}^{\prime \prime}\right|^{2} d t<\infty .
$$

(3) At any vertex $x \in \mathfrak{X}$, Kirchhoff's law

$$
\sum_{y:\{x, y\} \in \mathcal{E}} \mu_{x y} \vec{u}_{e_{x y}}(x)+\sum_{x \in \mathfrak{L}} \mu_{x x}\left(\vec{u}_{e_{x x}}(0)-\vec{u}_{e_{x x}}(\ell)\right)=0
$$

holds. Here, for $\{x, y\} \in \mathfrak{E}, \vec{u}_{e_{x y}}(x)$ is the (one-sided) derivative of $u$ at $x$ computed along $e_{x y}$ oriented from $x$ to $y$ and, for $x \in \mathfrak{L}, \vec{u}_{e_{x x}}(0)$ and $\vec{u}_{e_{x x}}(\ell)$ are the (one-sided) derivatives of $u_{e_{x x}}$ on $(0, \ell)_{x x}$ at 0 and at $\ell$.

We say that a function $u$ defined on a subset $\Omega$ is locally in $\mathcal{D}\left(\Delta_{\ell}\right)$ if it satisfies the above properties over $\Omega$ except for the global square integrable conditions on $u, u^{\prime}$ and $u^{\prime \prime}$. For such a function, $\Delta_{\ell} u$ is defined as the locally square integrable function $\Delta_{\ell} u=u^{\prime \prime}$ where $u^{\prime \prime}=u_{e_{x y}}^{\prime \prime}$ on $e_{x y} \cap \Omega$. 


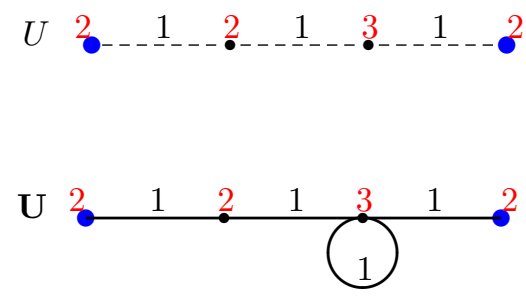

FigURE 8.20. $U$ and $\mathbf{U}$ in black with their boundaries in blue.

Remark 8.14. The stochastic process associated with the Dirichlet form $\mathcal{E}_{\mathbf{X}_{\ell}}$ can be explicitly constructed using Brownian motion. More specifically, starting at a vertex in the cable space, one performs Brownian excursions along adjacent edges until reaching another vertex. For a detailed description see Revuz and Yor (1999); Folz (2014). See Berkolaiko and Kuchment (2013) for a description of the related quantum graphs.

Definition 8.15. To any finite domain $U$ in $(\mathfrak{X}, \mathfrak{E})$ we associate the domain $\mathbf{U}=\mathbf{U}_{\ell}$ in $\mathbf{X}_{\ell}$ formed by all the vertices $x$ in $U$ and all the open edge $e_{x y}$ with at least one end point in $U$, including the loops $e_{x x}$ with $x \in U$.

See Figure 8.20 for an example of Defintion 8.15. As another example, consider the trivial finite domain $U=\{x\}$. To it, we associate the domain $\mathbf{U}$ formed by the vertex $x$ and all the open edges containing $x$, i.e., an open star around $x$, perhaps with a self-loop of length $\ell$, whose branches are in one to one correspondence with the $y \in \mathfrak{X}$ such that $\{x, y\} \in \mathfrak{E}$.

With this definition, the discrete finite domain $U$ is inner-uniform if and only if the domain $\mathbf{U}$ is inner-uniform in the metric space $\left(\mathbf{X}_{\ell}, \mathbf{d}_{\ell}\right)$. Following Gyrya and Saloff-Coste (2011, Definition 3.2) we say that a continuous domain $\mathbf{U}$ is inneruniform in the metric space $\left(\mathbf{X}_{\ell}, \mathbf{d}_{\ell}\right)$ if there exists constants $A^{c}$ and $\alpha^{c}$ such that, for each $\xi, \zeta \in \mathbf{U}$, there exists a continuous curve $\gamma_{\xi \zeta}:[0, \tau] \rightarrow \mathbf{U}$ (called an inner-uniform path) contained in $\mathbf{U}$ with $\left|\gamma_{\xi \zeta}\right|=\tau$ such that $(1) \gamma_{\xi \zeta}(0)=\xi$ and $\gamma_{\xi \zeta}(\tau)=\zeta,(2)\left|\gamma_{\xi \zeta}\right| \leq A^{c} \mathbf{d}_{\mathbf{U}}(\xi, \zeta)$ and (3) for any $t \in[0, \tau]$,

$$
\mathbf{d}_{\ell}\left(\gamma_{\xi \zeta}(t), \mathbf{X}_{\ell} \backslash \mathbf{U}\right) \geq \alpha^{c} \min \left\{t, \tau_{U}-t\right\}
$$

where $\mathbf{d}_{\mathbf{U}}$ is the distance in $\mathbf{U}$.

The important constants $A^{d}, \alpha^{d}$ and $A^{c}, \alpha^{c}$ ( $d$ for discrete, $c$ for continuous) capturing the key properties of an inner-uniform domain in both cases are within factors of 8 from each others. (This is because the discrete and continuous paths are not very different from each other, as explained below. Very large self-loops would be problematic, but we restrict to $\ell \in[0,1]$.) In fact, for any pair of points $\xi, \zeta$ in $\mathbf{U}$ we can define an inner-uniform path $\gamma_{\xi \zeta}$ from $\xi$ to $\zeta$ as follows. If the two points satisfy $\mathbf{d}_{\mathbf{U}}(\xi, \zeta)=\tau \leq 1$, i.e., they are either on the same edge or on two adjacent edges, then we set $\gamma_{\xi \zeta}$ to be the obvious path from $\xi$ to $\zeta$, parametrized by arc-length (one can easily check that this path satisfies $\mathbf{d}_{\ell}\left(\gamma_{\xi \zeta}(t), \mathbf{X}_{\ell} \backslash \mathbf{U}\right) \geq \min \{t, \tau-t\}$ ). When $\mathbf{d}_{\mathbf{U}}(\xi, \zeta)>1$, one can join them in $\mathbf{U}$ by first finding the closest points $x(\xi)$ and $x(\zeta)$ in $U$ (if there are multiple choices, pick one) and then use the obvious 
continuous extension of the discrete inner-uniform path from $x(\xi)$ to $x(\zeta)$, which is, again, parametrized by arc-length.

Finally we extend Definition 8.8 from $U$ to $\mathbf{U}$ as follows.

Definition 8.16. Let $U$ be a finite inner-uniform domain equipped with a central point $o \in U$ such that $d(o, \mathfrak{X} \backslash U)=\max \{d(x, \mathfrak{X} \backslash U): x \in U\}$. For any point $\xi \in \mathbf{U}$, let $\gamma_{\xi o}$ be the inner-uniform continuous path defined above joining $\xi$ to $o$ in $\mathbf{U}$. For any $\xi \in \mathbf{U}$ and $r>0$, let $\xi_{r}$ be defined by

$$
\xi_{r}=x(\xi)_{r} \text { if } r \geq 1
$$

where $x(\xi)$ is the (chosen) closest point to $\xi$ in $U$ and $x(\xi)_{r}$ is given by Definition 8.8, and

$$
\xi_{r}=\gamma_{\xi o}(\min \{r, \tau\}) \text { if } r \in(0,1) \text { and } \gamma_{\xi o}(\tau)=o, .
$$

Remark 8.17. The two key properties of the point $\xi_{r} \in \mathbf{U}$ are as follows. There are two constants $C, \epsilon$ which depends only on the inner-uniform constants $A, \alpha$ of $U$ such that

(1) The inner-distance $\mathbf{d}_{\mathbf{U}}\left(\xi, \xi_{r}\right)$ is no larger than $\mathrm{Cr}$;

(2) The distance $\mathbf{d}_{\ell}\left(\xi_{r}, X \backslash U\right)$ is at least $\epsilon r$.

In the present case, we chose the points $\xi_{r}$ so that, for $r \geq 1$, they actually belong to $U$ and coincide with $x(\xi)_{r}$ from Definition 8.8 .

The heat diffusion with Dirichlet boundary condition on the bounded inneruniform domain $\mathbf{U}=\mathbf{U}_{\ell}$ is studied in Lierl and Saloff-Coste (2014a,b). The heat diffusion semigroup with Dirichlet boundary condition on the domain $\mathbf{U}$ is the semigroup associated with the Dirichlet form obtained by closing the (closable) form

$$
\mathcal{E}_{\mathbf{U}, D}(f, f)=\int_{\mathbf{U}}\left|f^{\prime}\right|^{2} d \boldsymbol{\pi}
$$

defined on continuous functions $f$ in $\mathbf{U}$ that are locally in $\mathcal{D}\left(\mathcal{E}_{\mathbf{X}_{\ell}}\right)$ and have compact support in $\mathbf{U}$ (for such function, $f^{\prime}=f_{e_{x y}}^{\prime}$ on $e_{x y} \cap \Omega$ ). The subscript $D$ in this notation stands for Dirichlet condition. Let $H_{t}^{\mathbf{U}, D}=e^{t \Delta_{\mathbf{U}, D}}$ be the associated selfadjoint semigroup on $L^{2}\left(\mathbf{U}, \boldsymbol{\pi}_{\mathbf{U}}\right)$ with infinitesimal generator $\Delta_{\mathbf{U}, D}$. Here, $\boldsymbol{\pi}_{\mathbf{U}}$ is the normalized restriction of $\boldsymbol{\pi}$ to $\mathbf{U}$

$$
\boldsymbol{\pi}_{\mathbf{U}}=\left.\boldsymbol{\pi}(\mathbf{U})^{-1} \boldsymbol{\pi}\right|_{\mathbf{U}}
$$

The domain of $\Delta_{\mathbf{U}, D}$ is exactly the set of functions $f$ that are locally in $\mathcal{D}\left(\Delta_{\ell}\right)$ in $\mathbf{U}$, have limit 0 at the boundary points of $\mathbf{U}$ and satisfy $\int_{\mathbf{U}}\left|u^{\prime \prime}\right|^{2} d \boldsymbol{\pi}<\infty$. Also the parameter $\ell$ does not appear explicitly in the notation we just described, but all these objects depend on the choice of $\ell$.

Just as in the discrete setting, the key to the study of $H_{t}^{\mathbf{U}, D}$ is the Doobtransform technique which involves the positive eigenfunction $\phi_{\ell, 0}$ associated to the smallest eigenvalue $\boldsymbol{\lambda}_{\ell, 0}$ of $-\Delta_{\mathbf{U}, D}$ in $\mathbf{U}$. This function is defined by the following equations:

(1) $\boldsymbol{\lambda}_{\ell, 0}=\inf \left\{\int_{\mathbf{U}}\left|f^{\prime}\right|^{2} d \boldsymbol{\pi}_{\mathbf{U}}: f \in \mathcal{D}\left(\mathcal{E}_{\mathbf{U}, D}\right), \int_{\mathbf{U}}|f|^{2} d \boldsymbol{\pi}_{\mathbf{U}}=1\right\}$;

(2) $\boldsymbol{\phi}_{\ell, 0} \in \mathcal{D}\left(\Delta_{\mathbf{U}, D}\right)$ and $\Delta_{\mathbf{U}, D} \boldsymbol{\phi}_{\ell, 0}=-\boldsymbol{\lambda}_{l, 0} \boldsymbol{\phi}_{\ell, 0}$;

(3) $\int_{\mathbf{U}}\left|\phi_{\ell, 0}\right|^{2} d \boldsymbol{\pi}=1$. 
Proposition 8.18. Assume that $(\mathfrak{X}, \mathfrak{E}, \pi, \mu)$ is such that $\mu$ is adapted and $\mu$ is subordinated to $\pi$. Let $U$ be a finite domain in $(\mathfrak{X}, \mathfrak{E})$. There exists a value

$$
\ell_{0}=\ell_{0}(\mathfrak{X}, \mathfrak{E}, \pi, \mu, U) \in[0,1]
$$

of the loop-parameter $\ell$ such that the following properties hold true.

Let $\mathbf{U}$ be the bounded domain in $\mathbf{X}_{\ell_{0}}$ associated to $U$. Let $\phi_{0}, \beta_{0}$ be the PerronFrobenius eigenfunction and eigenvalue of $K_{U}$. Let $\boldsymbol{\phi}_{0}, \boldsymbol{\lambda}_{0}$ be the eigenfunction and bottom eigenvalue of $\Delta_{\mathbf{U}, D}$ for the parameter $\ell_{0}$ as defined above. There exists a constant $\kappa>0$ such that

(1) $\beta_{0}=\cos \left(\sqrt{\lambda_{0}}\right)$,

(2) $\phi_{0}(x)=\kappa \phi_{0}(x)$ for all vertices $x \in U$.

Proof: First, we study the function $\phi_{\ell, 0}$ for an arbitrary $\ell \in[0,1]$. On each edge $e_{x y}$ in $\mathbf{U}$, the function $\phi_{\ell, 0}$ satisfies

$$
\left(\frac{\partial}{\partial s}\right)^{2}\left[\phi_{\ell, 0}\right]_{e_{x y}}=-\boldsymbol{\lambda}_{\ell, 0}\left[\boldsymbol{\phi}_{\ell, 0}\right]_{e_{x y}}
$$

where $\left[\phi_{\ell, 0}\right]_{e_{x y}}$ is restriction of $\phi_{l, 0}$ to the edge $e_{x y}$. This implies

$$
\left[\phi_{\ell, 0}\right]_{e_{x y}}(s)=\frac{\phi_{\ell, 0}(y)-\cos \left(\sqrt{\boldsymbol{\lambda}_{\ell, 0}} \ell_{x y}\right) \phi_{\ell, 0}(x)}{\sin \left(\sqrt{\boldsymbol{\lambda}_{\ell, 0}} \ell_{x y}\right)} \sin \left(\sqrt{\boldsymbol{\lambda}_{\ell, 0}} s\right)+\phi_{\ell, 0}(x) \cos \left(\sqrt{\boldsymbol{\lambda}_{\ell, 0}} s\right)
$$

where $s \in\left(0, \ell_{x y}\right)$ parametrizes the arc length of $e_{x y}$ from $x$ to $y$ with

$$
\ell_{x y}= \begin{cases}1 & \text { when } x \neq y \\ \ell & \text { when } x=y\end{cases}
$$

When $x=y$,

$$
\left[\phi_{\ell, 0}\right]_{e_{x x}}(0)=\left[\phi_{\ell, 0}\right]_{e_{x x}}(\ell)=\phi_{\ell, 0}(x)
$$

and the function $\left[\phi_{\ell, 0}\right]_{e_{x x}}$ on the edge $(0, \ell)_{x x}$ satisfies

$$
\left[\boldsymbol{\phi}_{\ell, 0}\right]_{e_{x x}}(s)=\left[\boldsymbol{\phi}_{\ell, 0}\right]_{e_{x x}}(\ell-s) .
$$

To express Kirchhoff's law at $x \in U$, we compute, for $x \neq y$,

$$
\left[\overrightarrow{\boldsymbol{\phi}}_{\ell, 0}\right]_{e_{x y}}(0)=\frac{\sqrt{\boldsymbol{\lambda}_{\ell, 0}}}{\sin \left(\sqrt{\boldsymbol{\lambda}_{\ell, 0}}\right)}\left(\phi_{\ell, 0}(y)-\cos \left(\sqrt{\boldsymbol{\lambda}_{\ell, 0}}\right) \phi_{\ell, 0}(x)\right)
$$

and, for $x=y$,

$$
\begin{aligned}
{\left[\overrightarrow{\boldsymbol{\phi}}_{\ell, 0}\right]_{e_{x x}}(0)-\left[\overrightarrow{\boldsymbol{\phi}}_{\ell, 0}\right]_{e_{x x}}(1) } & =2\left[\overrightarrow{\boldsymbol{\phi}}_{\ell, 0}\right]_{e_{x x}}(0) \\
& =2 \frac{\sqrt{\boldsymbol{\lambda}_{\ell, 0}}}{\sin \left(\sqrt{\boldsymbol{\lambda}_{\ell, 0}}\right)}\left(1-\cos \left(\sqrt{\boldsymbol{\lambda}_{\ell, 0}} \ell\right)\right) \phi_{\ell, 0}(x) .
\end{aligned}
$$

It follows that Kirchhoff's law gives

$$
\begin{aligned}
\sum_{y:\{x, y\} \in \mathfrak{E}} \mu_{x y}\left(\phi_{\ell, 0}(y)\right. & \left.-\cos \left(\sqrt{\boldsymbol{\lambda}_{\ell, 0}}\right) \phi_{\ell, 0}(x)\right) \\
& +2 \mu_{x x} \frac{\sin \left(\sqrt{\boldsymbol{\lambda}_{\ell, 0}}\right)}{\sin \left(\sqrt{\boldsymbol{\lambda}_{\ell, 0}} \ell\right)}\left(1-\cos \left(\sqrt{\boldsymbol{\lambda}_{l, 0}} \ell\right)\right) \phi_{l, 0}(x)=0 .
\end{aligned}
$$


Recall that $K_{U}(x, y)=\mu_{x y} / \pi(x)$ for $x, y \in U$ with $\{x, y\} \in \mathfrak{E}$ and $K_{U}(x, x)=$ $\mu_{x x} / \pi(x)$. It follows that, for $x \in U$,

$$
K_{U} \boldsymbol{\phi}_{\ell, 0}(x)=\frac{1}{\pi(x)} \sum_{y \in U} \mu_{x y} \phi_{\ell, 0}(y)=\frac{1}{\pi(x)}\left(\sum_{y:\{x, y\} \in \mathfrak{E}} \mu_{x y} \boldsymbol{\phi}_{\ell, 0}(y)+\mu_{x x} \boldsymbol{\phi}_{\ell, 0}(x)\right),
$$

and Kirchhoff laws for $\phi_{\ell, 0}$ yields

$$
\begin{aligned}
& \frac{K_{U} \phi_{\ell, 0}(x)}{\phi_{\ell, 0}(x)}=K_{U}(x, x)+\left(1-K_{U}(x, x)\right) \cos \left(\sqrt{\boldsymbol{\lambda}_{\ell, 0}}\right) \\
& -2 K_{U}(x, x) \frac{\sin \left(\sqrt{\boldsymbol{\lambda}_{\ell, 0}}\right)}{\sin \left(\sqrt{\boldsymbol{\lambda}_{\ell, 0}} \ell\right)}\left(1-\cos \left(\sqrt{\boldsymbol{\lambda}_{\ell, 0}} \ell\right)\right) \\
& =\cos \left(\sqrt{\boldsymbol{\lambda}_{\ell, 0}}\right) \\
& +K_{U}(x, x)\left(1-\cos \left(\sqrt{\boldsymbol{\lambda}_{\ell, 0}}\right)\right)\left(1-2 \frac{\sin \left(\sqrt{\boldsymbol{\lambda}_{\ell, 0}}\right)}{\sin \left(\sqrt{\boldsymbol{\lambda}_{\ell, 0}}\right)} \frac{\left(1-\cos \left(\sqrt{\boldsymbol{\lambda}_{\ell, 0}}\right)\right)}{\left(1-\cos \left(\sqrt{\boldsymbol{\lambda}_{\ell, 0}}\right)\right)}\right)
\end{aligned}
$$

Given the uniqueness of the Perron-Frobenius eigenvalue and the fact that the associated positive eigenfunction is unique up to a multiplicative constant, the proposition follows from the previous computation if there exists $\ell_{0} \in[0,1]$ at which the function

$$
F(\ell)=1-2 \frac{\sin \left(\sqrt{\boldsymbol{\lambda}_{\ell, 0}}\right)}{\sin \left(\sqrt{\boldsymbol{\lambda}_{\ell, 0}} \ell\right)} \frac{\left(1-\cos \left(\sqrt{\boldsymbol{\lambda}_{\ell, 0}} \ell\right)\right)}{\left(1-\cos \left(\sqrt{\boldsymbol{\lambda}_{\ell, 0}}\right)\right)}
$$

vanishes. But, by an easy inspection, $F(0)=1$ and $F(1)=-1$. If we can prove that the function

$$
\ell \mapsto \lambda_{\ell, 0}
$$

is continuous, then $F$ must vanish somewhere between $l=0$ and $l=1$ by the intermediate value theorem, so we are done.

Fix $\ell_{1}, \ell_{2}$. Any function $f$ on $\mathbf{X}_{\ell_{1}}$ is turned into a function $\tilde{f}$ on $\mathbf{X}_{\ell_{2}}$ by setting

$$
\tilde{f}_{e_{x y}}(s)=\left\{\begin{array}{cl}
f_{e_{x y}}(s) & \text { if } x \neq y \\
f_{e_{x x}}\left(\ell_{2} s / \ell_{1}\right) & \text { if } x=y
\end{array}\right.
$$

Further,

$$
\int_{\mathbf{X}_{\ell_{2}}}|\tilde{f}|^{2} d \boldsymbol{\pi}=\int_{\mathbf{X}_{\ell_{1}}}|f|^{2} d \boldsymbol{\pi}+\left(\left(\ell_{1} / \ell_{2}\right)-1\right) \sum_{x \in \mathfrak{L}} \mu_{x x} \int_{e_{x x}}\left|f_{e_{x x}}\right|^{2} d t
$$

and

$$
\mathcal{E}_{\mathbf{X}_{\ell_{2}}}(\tilde{f}, \tilde{f})=\mathcal{E}_{\mathbf{X}_{\ell_{1}}}(f, f)+\left(\left(\ell_{2} / \ell_{1}\right)-1\right) \sum_{x \in \mathfrak{L}} \mu_{x x} \int_{e_{x x}}\left|f_{e_{x x}}^{\prime}\right|^{2} d t .
$$

Applying this to the function $\phi_{\ell_{1}, 0}$, normalized so that $\int_{\mathbf{X}_{\ell_{1}}}\left|\phi_{\ell_{1}, 0}\right|^{2} d \boldsymbol{\pi}=1$, we find that

$$
\boldsymbol{\lambda}_{\ell_{2}, 0} \leq \frac{\max \left\{1, \ell_{1} / \ell_{2}\right\}}{\min \left\{1, \ell_{2} / \ell_{1}\right\}} \boldsymbol{\lambda}_{\ell_{1}, 0} .
$$

Exchanging the role of $\ell_{1}, \ell_{2}$ yields the complementary inequality

$$
\boldsymbol{\lambda}_{\ell_{2}, 0} \geq \frac{\min \left\{1, \ell_{1} / \ell_{2}\right\}}{\max \left\{1, \ell_{2} / \ell_{1}\right\}} \boldsymbol{\lambda}_{\ell_{1}, 0} .
$$

This proves the continuity of $\ell \mapsto \boldsymbol{\lambda}_{\ell, 0}$ as desired. 
Remark 8.19. When the quantity $K_{U}(x, x)$ is constant, say, $K_{U}(x, x)=\theta$ for all $x \in U$, then every function $\phi_{\ell, 0}$ for $\ell \in[0,1]$ satisfies $\phi_{0}(x)=\kappa_{\ell} \phi_{\ell, 0}(x)$ at vertices $x \in U$, and we have

$$
\beta_{0}=1-\left(1-\cos \left(\sqrt{\boldsymbol{\lambda}_{\ell, 0}}\right)\right)\left(1-\theta\left(1-2 \frac{\sin \left(\sqrt{\boldsymbol{\lambda}_{\ell, 0}}\right)}{\sin \left(\sqrt{\boldsymbol{\lambda}_{\ell, 0} \ell}\right)} \frac{\left(1-\cos \left(\sqrt{\boldsymbol{\lambda}_{\ell, 0}} \ell\right)\right)}{\left(1-\cos \left(\sqrt{\boldsymbol{\lambda}_{\ell, 0}}\right)\right)}\right)\right)
$$

In words, the entire function of $\ell$ on the right-hand side is equal to the constant $\beta_{0}$.

Theorem 8.20 (Special case of Lierl and Saloff-Coste, 2014a, Proposition 5.10). Assume A1 with $\theta=2$ and fix $\alpha, A$. There exists a constant $C_{0}$ depending only on $\alpha, A, D, P_{e}, P$ such that, for any finite inner $(\alpha, A)$-uniform domain $U$ and loop parameter $\ell \in[0,1]$, the positive eigenfunction $\phi_{\ell, 0}$ for the $\Delta_{\mathbf{U}_{\ell, D}}$ in $\mathbf{U}_{\ell}$ is $\left(1 / 8, C_{0}\right)$ regular and satisfies

$$
\forall r>0, \xi \in \mathbf{U}_{\ell}, z \in B_{\mathbf{U}_{\ell}}(\xi, r / 2), \quad \phi_{\ell, 0}(z) \leq C_{0} \phi_{\ell, 0}\left(\xi_{r}\right) .
$$

Proof: The domain $\mathbf{U}=\mathbf{U}_{\ell}$ in $\left(\mathbf{X}_{\ell}, \mathbf{d}_{\ell}\right)$ is inner-uniform and the Dirichlet space $\left(\mathbf{X}_{\ell}, \boldsymbol{\pi}, \mathcal{E}_{\mathbf{X}_{\ell}}\right)$ is a Harnack space in the sense of Gyrya and Saloff-Coste (2011) and Lierl and Saloff-Coste (2014a). The most basic case of Lierl and Saloff-Coste (2014a, Proposition 5.10) provides the desired result. Technically speaking, the definition of the map $(x, r) \mapsto \xi_{r}$ here and in Lierl and Saloff-Coste (2014a) are slightly different but these differences are inconsequential.

Proof of Theorem 8.9: Together, Theorem 8.20 and Proposition 8.18 obviously yield Theorem 8.9.

Proof of Theorem 8.13: We use the same method as in the proof of Theorem 8.9 and extract this result from the similar result for the cable process with the proper choice $\ell_{0}$ of loop length. Local harmonic functions for the cable process (with Dirichlet boundary condition at the boundary of $U$ ) are always in a one-to-one correspondence with local harmonic functions for $K_{U}$, independently of the choice of the loop parameter $\ell$. Therefore, the stated result follows from Lierl and SaloffCoste (2014a, Theorem 5.5).

8.3. Point-wise kernel bounds. In this section, we describe how to obtain the following detailed point-wise estimates on the iterated kernels $K_{U}^{t}$ and $K_{\phi_{0}}^{t}$ when $U$ is inner-uniform. Recall that $V(x, r)=\pi(B(x, r))$ and $x_{\sqrt{t}}$ is a point such that $d\left(x_{\sqrt{t}}, \mathfrak{X} \backslash U\right) \geq \alpha(1+\sqrt{t})$ if $\sqrt{t} \leq R$ and $x_{\sqrt{t}}=o$ otherwise.

Theorem 8.21. Assume A1 with $\theta=2$ and fix $\alpha, A$. In addition, assume that the pair $(\pi, \mu)$ is such that $\sum_{y \in \mathfrak{X}} \mu_{x y} \leq(1-\epsilon) \pi(x)$ with $\epsilon>0$ (this means that $\left.\min _{x \in \mathfrak{X}}\left\{K_{\mu}(x, x)\right\} \geq \epsilon\right)$. There exist constants $c_{1}, c_{2}, C_{1}, C_{2} \in(0, \infty)$, which depend only on $\alpha, A, D, P_{e}, P$ and are such that, for any finite inner $(\alpha, A)$-uniform domain 
$U$, integer $t$ and $x, y \in U$ such that $d_{U}(x, y) \leq t$,

$$
\begin{aligned}
& \frac{C_{1} \exp \left(-c_{1} d_{U}(x, y)^{2} / t\right)}{\sqrt{V(x, \sqrt{t}) V(y, \sqrt{t})} \phi_{0}\left(x_{\sqrt{t}}\right) \phi_{0}\left(y_{\sqrt{t}}\right)} \\
& \leq \frac{K_{\phi_{0}}^{t}(x, y)}{\phi_{0}(y)^{2} \pi(y)} \\
& \leq \frac{C_{2} \exp \left(-c_{2} d_{U}(x, y)^{2} / t\right)}{\sqrt{V(x, \sqrt{t}) V(y, \sqrt{t})} \phi_{0}\left(x_{\sqrt{t}}\right) \phi_{0}\left(y_{\sqrt{t}}\right)} .
\end{aligned}
$$

Remark 8.22. When $t$ is larger than $R^{2}$ then $x_{\sqrt{t}}=o$ and the two-sided estimate above states that $K_{\phi_{0}}(x, y)$ is roughly of order $\pi_{\phi_{0}}(y)^{2} \pi_{U}(y)$ because $\phi_{0}(o)^{2} \simeq$ $\sum_{z \in U} \phi_{0}^{2}(z) \pi_{U}(z)=1$. The convergence result stated earlier give better estimates in this case. When $t \leq R^{2}$, the statement provides a useful estimate of the iterated kernel before equilibrium is reached.

The following corollary simply translates Theorem 8.21 in terms of the iterated kernel $K_{U}^{t}$.

Corollary 8.23. Assume A1 with $\theta=2$ and fix $\alpha, A$. In addition, assume that the pair $(\pi, \mu)$ is such that $\sum_{y \in \mathfrak{X}} \mu_{x y} \leq(1-\epsilon) \pi$ with $\epsilon>0$ (which implies that $\left.\min _{x \in \mathfrak{X}}\left\{K_{\mu}(x, x)\right\} \geq \epsilon\right)$. There exist constants $c_{1}, c_{2}, C_{1}, C_{2} \in(0, \infty)$ which depend only on $\alpha, A, D, P_{e}, P$ and are such that, for any finite inner $(\alpha, A)$-uniform domain $U$, for any integer $t$ and any $x, y \in U$ such that $d_{U}(x, y) \leq t$,

$$
\begin{aligned}
& \frac{C_{1} \beta_{0}^{t} \phi_{0}(x) \phi_{0}(y) \exp \left(-c_{1} d_{U}(x, y)^{2} / t\right)}{\sqrt{V(x, \sqrt{t}) V(y, \sqrt{t})} \phi_{0}\left(x_{\sqrt{t}}\right) \phi_{0}\left(y_{\sqrt{t}}\right)} \\
& \leq \frac{K_{U}^{t}(x, y)}{\pi(y)} \\
& \leq \frac{C_{2} \beta_{0}^{t} \phi_{0}(x) \phi_{0}(y) \exp \left(-c_{2} d_{U}(x, y)^{2} / t\right)}{\sqrt{V(x, \sqrt{t}) V(y, \sqrt{t})} \phi_{0}\left(x_{\sqrt{t}}\right) \phi_{0}\left(y_{\sqrt{t}}\right)}
\end{aligned}
$$

Outline of the proof of Theorem 8.21: To simplify notation, set

$$
\widetilde{K}=K_{\phi_{0}}, \quad \widetilde{\pi}=\left.\phi_{0}^{2} \pi\right|_{U} .
$$

The estimates stated above and which we are going to obtain for $\widetilde{K}^{t}=K_{\phi_{0}}^{t}$ do not depend on the exact scaling of $\phi_{0}$ and $\left.\pi\right|_{U}$ as long as the given choice made is used consistently. The first key point of the proof is the fact that $\widetilde{K}=K_{\phi_{0}}$ is Markov (i.e., satisfies $\sum_{y \in U} \widetilde{K}(x, y)=1$ for each $x \in U$ ) and reversible with respect to $\widetilde{\pi}=\left.\phi_{0}^{2} \pi\right|_{U}$. (Normalizing is optional.) Also, the reversible Markov chain $(\widetilde{K}, \widetilde{\pi})$ satisfies $\widetilde{K}(x, x) \geq \epsilon$ and the ellipticity condition $\widetilde{K}(x, y) \geq 1 / \widetilde{P}_{e}$ where $\widetilde{P}_{e}=\beta_{0}^{-1} P_{e} \max \left\{\phi_{0}(x) / \phi_{0}(y):\{x, y\} \in \mathfrak{E}_{U}\right\}$. The constant $\widetilde{P}_{e}$ is bounded above in terms of the constants $\alpha, A, D, P, P_{e}, \epsilon$ only.

It is well-known (see Barlow, 2017, Theorem 6.34 or Delmotte, 1999) that the two-sided Gaussian-type estimate stated in Theorem 8.21 for the reversible Markov chain $(\widetilde{K}, \widetilde{\pi})$ is equivalent to the conjunction of two more geometric properties which are (a) the doubling property

$$
\forall x \in U, r>0, \quad \widetilde{V}(x, 2 r) \leq \widetilde{D} \widetilde{V}(x, r)
$$


of the volume function

$$
\widetilde{V}(x, r)=\widetilde{\pi}\left(B_{U}(x, r)\right)=\left.\sum_{y \in B_{U}(x, r)} \phi_{0}^{2}(y) \pi\right|_{U}(y),
$$

and (b) the Poincaré inequality

$$
\min _{\xi} \sum_{B_{U}(x, r)}|f(y)-\xi|^{2} \widetilde{\pi}(y) \leq \widetilde{P} r^{2} \sum_{y, z \in B_{U}(x, r)}|f(y)-f(z)|^{2} \widetilde{K}(z, y) \widetilde{\pi}(z),
$$

for all $x \in U, r>0$ and all $f$ defined over $B_{U}(x, r)$. See Delmotte (1999).

Theorem 8.9 shows that

$$
\widetilde{V}(x, r) \simeq \phi_{0}\left(x_{r}\right)^{2} V(x, r)
$$

and the doubling property of $\widetilde{V}$ follows from Corollary 8.10. The proof of the Poincare inequality on the balls $B_{U}(x, r)$ follows from a variation on the argument developed in Section 4 which uses the additional property of inner-uniform domains. See Gyrya and Saloff-Coste (2011) for the proof in the context of strictly local Dirichlet spaces and Houston-Edwards (2018) for the case of discrete graphs.

The following useful corollary to Theorem 8.21 is illustrated in several different examples in Section 9.

Corollary 8.24. Given the setup of Theorem 8.21,

$$
c \beta_{0}^{t} \frac{\phi_{0}(x)}{\phi_{0}\left(x_{\sqrt{t}}\right)} \leq \mathbf{P}_{x}\left(\tau_{U}>t\right) \leq C \beta_{0}^{t} \frac{\phi_{0}(x)}{\phi_{0}\left(x_{\sqrt{t}}\right)},
$$

where $\tau_{U}$ is the first time that the process $\left(X_{t}\right)$ exits $U$, and $c, C>0$ are constants which depend only on $\alpha, A, D, P_{e}, P$.

Proof: Remark 8.17 gives us a constant $c$ such $d\left(x_{r}, \mathfrak{X} \backslash U\right) \geq c r$. Note that for any $y \in B\left(x_{\sqrt{t}}, c \sqrt{t} / 2\right)$, we have $\phi_{0}(y) \leq C \phi_{0}\left(x_{\sqrt{t}}\right)$ and $\phi_{0}\left(y_{\sqrt{t}}\right) \geq C^{-1} \phi_{0}\left(x_{\sqrt{t}}\right)$. Furthermore, Theorem 8.9 gives that

$$
\widetilde{V}(x, \sqrt{t}) \approx \widetilde{V}(y, \sqrt{t}) \approx V\left(x_{\sqrt{t}}, c \sqrt{t} / 2\right) \approx \phi_{0}\left(x_{\sqrt{t}}\right)^{2} V(x, \sqrt{t}) .
$$

Now, we use the lower bound concerning $K_{U}^{t}$ from Corollary 8.23 and the previous observations to obtain

$$
\begin{aligned}
\mathbf{P}_{x}\left(\tau_{U}>t\right) & =\sum_{y \in U} K_{U}^{t}(x, y) \geq \sum_{y \in B(x \sqrt{t}, c \sqrt{t} / 2)} K_{U}^{t}(x, y) \\
& \geq c_{1}^{\prime} \beta_{0}^{t} \frac{\phi_{0}(x)}{\phi_{0}\left(x_{\sqrt{t}}\right)} .
\end{aligned}
$$

For the upper bound, also using Corollary 8.23,

$$
\begin{aligned}
\mathbf{P}_{x}\left(\tau_{U}>t\right) & =\sum_{y \in U} K_{U}^{t}(x, y) \\
& \leq C_{2} \beta_{0}^{t} \frac{\phi_{0}(x)}{\phi_{0}\left(x_{\sqrt{t}}\right)} \sum_{y \in U} \frac{\phi_{0}(y)}{\phi_{0}\left(y_{\sqrt{t}}\right)} \frac{e^{-c_{2} d_{U}^{2}(x, y)}}{\sqrt{V(x, \sqrt{t}) V(y, \sqrt{t})}} \pi(y) \\
& \leq C_{2}^{\prime} \beta_{0}^{t} \frac{\phi_{0}(x)}{\phi_{0}\left(x_{\sqrt{t}}\right)} .
\end{aligned}
$$


The last inequality holds because $\phi_{0}(y) \leq C \phi_{0}\left(y_{\sqrt{t}}\right)$ by Theorem 8.9, and

$$
\sum_{y \in U} \frac{e^{-c_{2} d_{U}^{2}(x, y)}}{\sqrt{V(x, \sqrt{t}) V(y, \sqrt{t})}} \pi(y) \leq C
$$

on any doubling space.

\section{Some explicit examples}

In this section, we consider explicit families of finite domains indexed by a size parameter $N$ which is comparable to the diameter of the relevant domain. Each finite domain $U$ is an $\alpha$-inner-uniform domain with a chosen "center" $o$ which is just a point in $U$ at maximal distance $R=R_{U}$ from the boundary (see Lemma 8.3). Within each family, the inner-uniformity parameter, $\alpha \in(0,1)$, is fixed.

The underlying weighted graph $(\mathfrak{X}, \mathfrak{E}, \pi, \mu)$ for these examples satisfies A1 with $\theta=2$. In fact, in this section, the underlying space is the square grid $\mathbb{Z}^{d}$ of some fixed dimension $d$ (or some simple modification of it).

We normalize the Perron-Frobenius eigenfunction $\phi_{0}$ by $\pi_{U}\left(\phi_{0}^{2}\right)=1$. Because of Theorem 8.9, we have

$$
\max \left\{\phi_{0}\right\} \leq C_{0} \phi_{0}(o)
$$

and (see the $\left(1 / 8, C_{0}\right)$-regularity of $\left.\phi_{0}\right)$,

$$
C_{0} \min _{B(o, R / 2)}\left\{\phi_{0}\right\} \geq \phi_{0}(o) .
$$

Furthermore, $\pi_{U}(B(o, R / 2)) \geq c_{0} \pi(U)$. It follows that

$$
\forall y \in B(o, R / 2), \quad \phi_{0}(y) \approx \phi_{0}(o) \simeq 1
$$

uniformly within each family of examples considered. In fact, in many examples, the choice of the point $o$ is somewhat arbitrary because one could as well pick any point $\tilde{o}$ with the property that

$$
d(\tilde{o}, \mathfrak{X} \backslash U) \geq \frac{1}{2} \max _{x \in U}\{d(x, \mathfrak{X} \backslash U)\}=\frac{R}{2} .
$$

Any such point $\tilde{o}$ has the property that

$$
\forall y \in B(\tilde{o}, R / 4), \quad \phi_{0}(y) \approx \phi_{0}(\tilde{o}) \approx \phi_{0}(o) \simeq 1
$$

uniformly over $\tilde{o}$ and within each family of examples considered. See Figure 9.21.
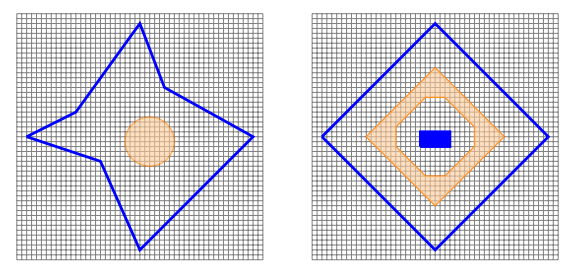

FiguRE 9.21. In light orange, regions where $\phi_{0}$ is approximately equal to 1 . On the left, an example in which there is essentially one central point $o$. On the right, an example in which the "center" $o$ can be placed in a variety of different location. 


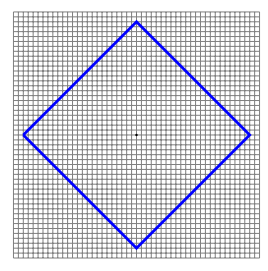

Figure $9.22 . \quad B(N)$ in $\mathbb{Z}^{2}$

9.1. Graph distance balls in $\mathbb{Z}^{2}$. In $\mathbb{Z}^{2}$, let $U=B(N)=\left\{x=(p, q) \in \mathbb{Z}^{2}:|p|+|q| \leq\right.$ $N\}$. This is the graph ball around 0 in $\mathbb{Z}^{2}$. Equip $\mathbb{Z}^{2}$ with the counting measure $\pi$ and with edge weights

$$
\mu_{x y}= \begin{cases}1 / 8 & \text { if }\left|p_{x}-p_{y}\right|+\left|q_{x}-q_{y}\right|=1 \\ 0 & \text { otherwise. }\end{cases}
$$

The Markov kernel $K_{\mu}$ drives a lazy random walk on the square lattice, with holding probability $1 / 2$ at each vertex. We are interested in the kernel

$$
K_{U}(x, y)=K_{\mu}(x, y) \mathbf{1}_{U}(x) \mathbf{1}_{U}(y)
$$

which we view as defining an operator on $L^{2}\left(U, \pi_{U}\right)$ where $\pi_{U}$ is the uniform probability measure on $U$. This set is clearly inner-uniform (in fact, it is uniform because the inner distance between any two points in $U$ is the same as the distance between these point in $\mathbb{Z}^{2}$ ).

Let us introduce the Perron-Frobenius eigenfunction $\phi_{0}$ and its eigenvalue $\beta_{0}$. Obviously, they depend on $N$. This is one of the rare cases when $\phi_{0}$ and $\beta_{0}$ can be determined explicitly:

$$
\phi_{0}(x)=\kappa_{N} \cos \left(\frac{\pi}{2(N+1)}(p+q)\right) \cos \left(\frac{\pi}{2(N+1)}(p-q)\right)
$$

with

$$
\beta_{0}=\frac{1}{2}\left(1+\cos ^{2}\left(\frac{\pi}{2(N+1)}\right)\right)
$$

The normalizing constant $\kappa_{N}$ is of order 1 . Here we need to recall that $\phi_{0}$ vanishes on points at graph distance $N+1$ from the origin in $\mathbb{Z}^{2}$.

To illustrate our result for estimating $\mathbf{P}_{x}\left(\tau_{U}>t\right)$ without writing long formulas, let us consider the probabilities $\mathbf{P}_{(p, 0)}\left(\tau_{U}>t\right)$ and $\mathbf{P}_{(p, p)}\left(\tau_{U}>t\right)$ that a random walk started at $x=(p, 0)$ (for $0 \leq p \leq N)$ and $x=(p, p)$ (for $0 \leq p \leq N / 2$ ), respectively, has not yet been killed by time $t$. For all $t \leq N^{2}$, we have

$$
\mathbf{P}_{(p, 0)}\left(\tau_{U}>t\right) \approx\left(\frac{N-p}{N-p+\sqrt{t}}\right)^{2}, 0 \leq p \leq N .
$$


This comes from applying Corollary 8.24 to the eigenfunction above,

$$
\begin{aligned}
\mathbf{P}_{(p, 0)}\left(\tau_{U}>t\right) & \approx \frac{\phi_{0}((p, 0))}{\phi_{0}((p, 0) \sqrt{t})} \\
& \approx \frac{\phi_{0}((p, 0))}{\phi_{0}((p-\sqrt{t}, 0))} \\
& \approx \frac{\left(\cos \left(\frac{\pi}{2 N} p\right)\right)^{2}}{\cos \left(\frac{\pi}{2 N}(p-\sqrt{t})\right)^{2}} .
\end{aligned}
$$

Now, use that $\cos \left(\frac{\pi}{2 N} x\right)=\sin \left(\frac{\pi}{2 N}(N-x)\right) \sim \frac{\pi}{2 N}(N-x)$. In particular, for any fixed $0<t \leq N^{2}, P_{(p, 0)}\left(\tau_{U}>t\right)$ vanishes asymptotically like $\frac{(N-p)^{2}}{t}$ as $p$ tends to $N$.

Similarly, for $0<t \leq N^{2}$,

$$
\mathbf{P}_{(p, p)}\left(\tau_{U}>t\right) \approx\left(\frac{N-2 p}{N-2 p+\sqrt{t}}\right), 0 \leq 2 p \leq N .
$$

In this case, for any fixed $0<t \leq N^{2}, \mathbf{P}_{(p, p)}\left(\tau_{U}>t\right)$ vanishes like $\frac{N-2 p}{\sqrt{t}}$ when $p$ tends to $N / 2$.

Remark 9.1. While our results apply equally well to the graph distance balls of $\mathbb{Z}^{d}$ for $d>2$, they are much more complicated in that case and there is no explicit formula for $\phi_{0}$ or the eigenvalue $\beta_{0}$. The ball is a polytope with faces of dimension $0,1, \ldots, d$. The vanishing of $\phi_{0}$ near each of these faces is described by a power function of the distance to the particular face that is considered. The exponent depends on the dimension of the face and on the angles made by the higher dimensional faces meeting at the given face (the exponent is always 1 when approaching the highest dimensional faces).

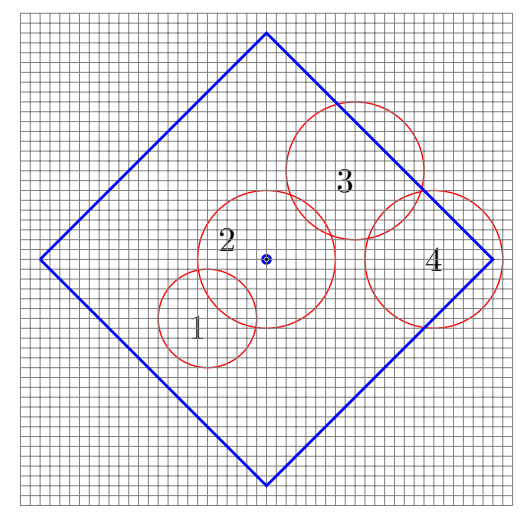

FiguRe 9.23. $B(N) \backslash\{0\}$ in $\mathbb{Z}^{2}$ (the blue central point is part of the boundary)

9.2. $B(N) \backslash\{(0,0)\}$ in $\mathbb{Z}^{2}$. The case when $U=B(N) \backslash\{(0,0)\}$ is interesting because we are able to describe precisely the behavior of $\phi_{0}$ even though there is no explicit formula available. First, we note again that this is an inner-uniform domain (there is no preferred point $o$ in this case, since any point at distance of order $N / 2$ from 
$(0,0)$ will do). Theorem 8.13 will play a key part in allowing us to describe the behavior of $\phi_{0}$. First, we claim that

$$
1-\beta_{0} \approx N^{-2} \text {. }
$$

The lower bound follows from comparison with the eigenvalue bound from the box itself $B(N)$ and because $\beta_{0}$ is increasing under inclusion. For the upper bound, use the test function

$$
f((p, q))=\min \{d((0,0),(p, q)), N+1-d((0,0),(p, q))\}
$$

which vanishes at all boundary points for $U$.

Second, we show that

$$
\phi_{0}((p, q)) \approx \frac{(N-|p+q|)(N-|p-q|)) \log (1+|p|+|q|)}{N^{2} \log N} .
$$

To obtain this result, cover $U$ by a finite number (independent of $N$ ) of $\mathbb{Z}^{2}$ balls $\left\{B_{j}\right\}$ of radius of order $N$ so that the trace of $U$ in each of the balls $2 B_{j}$ is of one of the following four types: (1) no intersection with the boundary of $U ;(2)$ the intersection with the boundary of $U$ is $\{(0,0)\}$; (3) the intersection with the boundary of $U$ is a subset of $\{(p, q): p+q=N\},\{(p, q): p-q=N\},\{(p, q): p+q=-N\}$, or $\{(p, q): p-q=-N\}$; and (4) the intersection with the boundary is a corner formed by two of the previously mentioned lines. See Figure 9.23 for an illustration of these four types. In case (1), we know that $\phi$ is approximately constant in $B_{j}$. Moreover, this approximately constant value must be (approximately) the maximum value of $\phi_{0}$ because of Theorem 8.9, and this constant must be approximatively equal to 1 because $\phi_{0}$ is normalized by $\pi_{U}\left(\phi_{0}^{2}\right)=1$. This is compatible with the proposed formula describing $\phi_{0}$. In case (2), Theorem 8.13 allows us to compare $\phi_{0}((p, q))$ to the harmonic function $h((p, q))$ equal to the discrete modified Green's function (or potential kernel),

$$
A((0,0),(p, q))=\sum_{t=0}^{\infty}\left(M^{t}((0,0),(p, q))-M^{t}((0,0),(0,0))\right)
$$

on $\mathbb{Z}^{2} \backslash\{(0,0)\}$. Here $M$ is the Markov kernel of aperiodic simple random walk on $\mathbb{Z}^{2}$. It is well-known that this function is comparable to $\log (1+|p|+|q|)$ (See Spitzer, 1976, Chapter 3 from which we borrowed the notation $A(x, y)$. More precise estimates are available using a sharp version of the local limit theorem, but this is enough for our purpose). Because the ball $B_{j}$ in question must contain a point at distance of order $N$ from the boundary of $U$ at which $\phi_{0}$ is of order 1, we find that, in such a ball,

$$
\phi_{0}((p, q)) \approx \frac{\log (1+|p|+|q|)}{\log N} .
$$

Again, this estimate is compatible with the proposed formula. In case (3), we easily have a linear function $h$ vanishing on the (flat) portion of the boundary contains in that ball and positive discrete harmonic in $U$. Thanks to Theorem 8.13, this provides the estimate

$$
\phi_{0}((p, q)) \approx \frac{\left.d_{U}((p, q)), \mathfrak{X} \backslash U\right)}{N}
$$

in balls of this type, which has the form suggested by the proposed formula. Finally, in case (4), and, for definiteness, in the case the ball $B_{j}$ is centered at the corner of intersection of the line $\{(p, q): p+q=N\}$ and $\{(p, q): p-q=N\}$, the function 
$h((p, q))=(N-p-q)(N-p+q)$ vanishes on these two lines and is discrete harmonic. This gives (again, using Theorem 8.13)

$$
\phi_{0}((p, q)) \approx \frac{(N-p-q)(N-p+q)}{N^{2}}
$$

as desired.

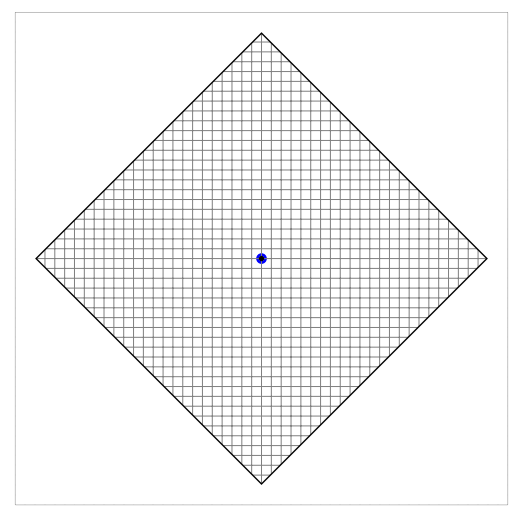

Figure 9.24. $B(N) \backslash\{0\}$ in $B(N)$ (The blue central point is the entire boundary.)

9.3. $B(N) \backslash\{\mathbf{0}\}$ in $B(N)$, in dimension $d>1$. First we explain the title of this subsection. Consider the simple random walk in the ball $B(N) \subset \mathbb{Z}^{d}$, with any reasonable reflection type hypothesis on the boundary of $B(N)$. Our aim is to study absorption at 0 for this random walk on the finite set $B(N)$. To put this example in our general framework, we set $\mathfrak{X}_{N}=B(N)$ equipped with the edge set $\mathfrak{E}_{N}$ induced by the underlying square lattice, that is the collection of all lattice edges with both end points in $B(N)$. The measure $\pi$ on $\mathfrak{X}_{N}=B(N)$ is the counting measure and each lattice edge $e$ in $\mathfrak{E}$ is given the weight $\mu(e)=1 /(2 d)$. This means that the Markov kernel $K_{\mu}$ for our underlying walk has no holding at point $x \in B(N-1) \subset B(N)$ and holding probability $\nu(x) /(2 d)$ where $\nu(x)=2 d-\#\{y \in$ $\left.B(N):\{x, y\} \in \mathfrak{E}_{N}\right\}$ when $x \in B(N) \backslash B(N-1)$ (this holding probability at the boundary is always at least $1 / 2)$. The domain $U_{N}$ of interest to us here is $U_{N}=B(N) \backslash\{\mathbf{0}\}$ (inside $B(N)$ ) whose sole outside boundary point is the center $\mathbf{0}$. When the dimension $d$ is at least 2 , this is an inner-uniform domain in $\left(\mathfrak{X}_{N}, \mathfrak{E}_{N}\right)$ (there is no canonical center but any point at distance at least $N / 2$ from $\mathbf{0}$ can be chosen to be the center $o$ ).

Because the domain $U_{N}$ is inner-uniform (uniformly in $N$ ), Theorem 8.13 yields

$$
\mathbf{P}_{x}\left(\tau_{U_{N}}>t\right) \approx \frac{\beta_{0}^{t} \phi_{0}(x)}{\phi_{0}\left(x_{\sqrt{t}}\right)}
$$

and, for $t \geq N^{2}$, Corollary 8.11 gives,

$$
\left.\left|K_{U_{N}}^{t}(x, y)-\phi_{0}(x) \phi_{0}(y) \beta_{0}^{t}\right| U_{N}\right|^{-1} \mid \leq C \beta_{0}^{t} \phi_{0}(x) \phi_{0}(y) e^{-t / N^{2}} \text {. }
$$

As in the previous examples, the key is to obtain further information on $\beta_{0}$ and $\phi_{0}$. For that we need to treat the cases $d=2$ and $d>2$ separately. In both cases, we use Theorem 8.13 to estimate $\phi_{0}$. 
9.3.1. Case $d=2$. The first task is to estimate $1-\beta_{0}$ from above and below. This is done by using the same argument explained in Saloff-Coste (1997, Example 3.2.5: The dog). See Subsection 9.4.1 below where we spell out the main part of the argument in question. The upshot is that $1-\beta_{0} \approx 1 /\left(N^{2} \log N\right)$. We know that $\phi_{0}(x) \approx 1$ when $x$ is at graph distance at least $N / 2$ from $\mathbf{0}$ (see the outline described in Example 9.3 for type 1 balls). To estimate $\phi_{0}$ at other points, we compare it with the global positive harmonic function from $\mathbb{Z}^{2} \backslash\{\mathbf{0}\}$ given by the so-called modified Green's function $h(x)=A(\mathbf{0}, x)=\sum_{t=0}^{\infty}\left[M^{t}(\mathbf{0}, x)-M^{t}(\mathbf{0}, \mathbf{0})\right]$ where $M$ stands here for the Markov kernel of aperiodic simple random walk in $\mathbb{Z}^{2}$ as in Example 9.3. Note that $h$ vanishes at 0. Classical estimates (e.g., Spitzer, 1976 ) yield $h(x) \approx \log |x|$. This, together with Theorem 8.13 and the estimate when $x$ is at distance at least $N / 2$ from $\mathbf{0}$, gives

$$
\phi_{0}(x) \approx \frac{\log |x|}{\log N}
$$

9.3.2. Case $d>2$. The case $d>2$ is perhaps easier although the arguments are essentially the same. The eigenvalue $\beta_{0}$ is estimated by $1-\beta_{0} \approx 1 / N^{d}$ and the harmonic function $h(x)=\sum_{t=0}^{\infty} M^{t}(\mathbf{0}, x)-\sum_{t=0}^{\infty} M^{t}(\mathbf{0}, \mathbf{0})$ (these sums converge separately because $d>2)$ is estimated by $h(x) \approx\left(1-1 /(1+|x|)^{d-2}\right)$. This gives

$$
\phi_{0}(x) \approx \frac{\left(1-1 /(1+|x|)^{d-2}\right)}{\left(1-1 /(1+N)^{d-2}\right)} \approx 1 .
$$

9.3.3. Discussion. The first thing to observe in these examples is the fact that $1-\beta_{0}=o\left(1 / N^{2}\right)$. For $t \geq N^{2}$ we have

$$
\left.\left|K_{U}^{t}(x, y)-\phi_{0}(x) \phi_{0}(y) \beta_{0}^{t}\right| U\right|^{-1} \mid \leq C \beta_{0}^{t} \phi_{0}(x) \phi_{0}(y) e^{-t / N^{2}} .
$$

In the case $d=2$, if $\epsilon>0$ is fixed and $x, y$ are at distance greater than $N^{\epsilon}$ from the origin, we can without loss of information, simplify the above statement and write

$$
\left.\left|K_{U}^{t}(x, y)-\phi_{0}(x) \phi_{0}(y) \beta_{0}^{t}\right| U\right|^{-1} \mid \leq C e^{-t / N^{2}}
$$

Because $\beta_{0}^{t}$ decays significantly slower than $e^{-t / N^{2}}$, this provides a good example of a quasi-stationary distribution during the time interval $t \in\left(N^{2}, N^{2} \log N\right)$.

In the case $d>2$, the same phenomenon occurs, only in an even more tangible way. For any $x, y \in U_{N}, \phi_{0}(x), \phi_{0}(y)$ are uniformly bounded away from 0 (even for the neighbors of the origin, $\mathbf{0})$. Moreover, $1-\beta_{0} \approx 1 / N^{d}=o\left(1 / N^{2}\right)$. For $t \geq N^{2}$ and $x, y \in U_{N}$,

$$
\left.\left|K_{U}^{t}(x, y)-\phi_{0}(x) \phi_{0}(y) \beta_{0}^{t}\right| U\right|^{-1} \mid \leq C e^{-t / N^{2}}
$$

For any fixed $T$ and all $N$ bigger than $T^{2 /(d-2)}$, on intervals of the type $t \in$ $\left(T N^{2}, N^{d} / T\right), K_{U}^{t}(x, y)$ is well approximated by $\phi_{0}(x) \phi_{0}(y) \mid U^{-1}$ because, on such intervals, $\beta_{0}^{t}$ remains close to 1 .

9.4. $B(N) \backslash B_{2}(L)$ in $B(N)$, in dimension $d>1$. We work again in $\mathfrak{X}_{N}=B(N)$ with the weighted graph structure explained above. We use $B_{2}(r)$ to denote the trace on the lattice $\mathbb{Z}^{d}$ of the Euclidean (round) ball of radius $r$ centered at the origin, 0. The domain we wish to investigate is $U_{N, L}=B(N) \backslash B_{2}(L)$ with $L=o(N)$ so that the number of points in $U_{N, L}$ is of order $N^{d}$ and $U_{N, L}$ is inner-uniform (uniformly in all choices of $N, L$ ). Again, the chosen center $o$ in $U_{N, L}$ can be any 
point at graph distance $N$ from $\mathbf{0}$. All the estimates described below are uniform in $N, L$ as long as $L=o(N)$.

9.4.1. Estimating $\beta_{0}$. First we explain how to estimate $\beta_{0}$ for $U_{N, L}=B(N) \backslash B_{2}(L)$ in $B(N)$ using and argument very similar to those used in Saloff-Coste (1997, Example 3.2.5: The dog). For each point $x \in U$ fix a graph geodesic discrete path $\gamma_{x}$ that joins $x$ to the origin in $\mathbb{Z}^{d}$ while staying as close as possible to the straight line from $x$ to the origin. We stop $\gamma_{x}$ whenever it reaches a point in $B_{2}(L)$.

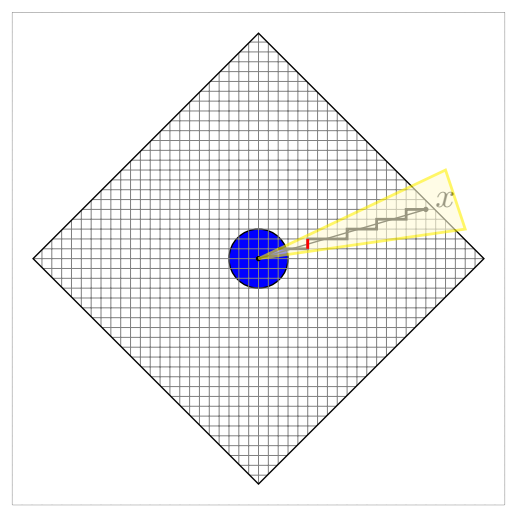

Figure 9.25. Paths to the origin in $B(N) \backslash B_{2}(L)$

Given a function $f$ on $B(N)$ which is equal to zero on $B_{2}(L)$ and a directed edge $e=(x, y)$, set $d f(e)=f(y)-f(x)$. The edges along a path $\gamma_{x}$ are all directed toward the origin. Using this notation, we have

$$
|f(x)|^{2} \leq\left|\sum_{e \in \gamma_{x}} d f(e)\right|^{2} \leq\left|\gamma_{x}\right|_{w} \sum_{e \in \gamma_{x}}|d f(e)|^{2} w(e)
$$

where $w$ is a weight function on the edge $e$ which will be chosen later and $|\gamma|_{w}=$ $\sum_{e \in \gamma} w(e)^{-1}$. Summing over all $x \in U$, we obtain

$$
\sum_{x \in U}|f|^{2} \leq 2 d \sum_{e \in \mathfrak{E}}\left(\sum_{x: \gamma_{x} \ni e}\left|\gamma_{x}\right|_{w} w(e)\right) \frac{|d f(e)|^{2}}{2 d} \leq C_{w}(d, N, L) \mathcal{E}_{\mu}(f, f)
$$

where

$$
C_{w}(d, N, L)=2 d \max _{e \in \mathbb{E}}\left\{w(e) \sum_{x: \gamma_{x} \ni e}\left|\gamma_{x}\right|_{w}\right\} .
$$

Using the Rayleigh quotient formula for $1-\beta_{0}$, we obtain the eigenvalue estimate

$$
\beta_{0} \leq 1-1 / C_{w}(d, N, L)
$$

for any choice of the weight $w$. Here we choose $w(e)$ to be the Euclidean distance of the edge $e$ to the origin raised to the power $d-1$. This implies that

$$
\left|\gamma_{x}\right|_{w} \leq C_{d} \times \begin{cases}\log (N / L) & \text { when } d=2 \\ L^{-d+2} & \text { when } d>2\end{cases}
$$


for some constant $C_{d}$ which depends on the dimension $d$. It remains to count how many $x$ use a given edge $e$. Because we use paths that remain close to the straight line from $x$ to the origin, the vertices $x$ that use and given edge $y$ at Euclidean distance $T$ from the origin must be in a cone of aperture bounded by $C_{d} / T$. The number of these vertices is at most $C_{d} N \times(N / T)^{d-1}$ where the constant $C_{d}$ changes from line to line. See Figure 9.25. Recall that $w(e) \approx T^{d-1}$. Putting things together yields

$$
C_{w}(d, N, L) \leq C_{d} \times \begin{cases}N^{2} \log (N / L) & \text { when } d=2, \\ N^{d} L^{-d+2} & \text { when } d>2 .\end{cases}
$$

In terms $\beta_{0}$ this gives

$$
1-\beta_{0} \geq C_{d}^{-1} \times \begin{cases}1 / N^{2} \log (N / L) & \text { when } d=2 \\ L^{d-2} / N^{d} & \text { when } d>2\end{cases}
$$

The upper-bound is a simple computation using a test function which take the value 0 on $B_{2}(L)$ and increase linearly at rate 1 until taking the value $L$. After that the test function remains constant equal to $L$. Note that this bound interpolates between the case $L=1$ (more or less, the previous case) when $1-\beta_{0} \approx 1 / N^{d}$ and the case when $L$ is a fixed small fraction of $N$, in which case $1-\beta_{0} \approx 1 / N^{2}$.

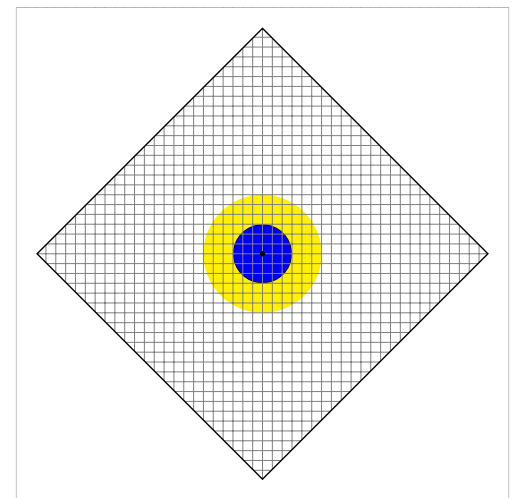

FiguRE 9.26. $U_{N, L}=B(N) \backslash B_{2}(L)$ : In the yellow region of width $L$ around $B_{2}(L), \phi_{0}(x) \approx\left(\frac{\log L}{\log N}\right) d\left(x, B_{2}(L)\right)$.

9.4.2. Estimating $\phi_{0}$ in the case $d=2$. The technique is the same as the one described below for the case $d>2$. Here we omit the details and only describe the findings. The behavior of the function $\phi_{0}$ is best described by considering two zones. See Figure 9.26. The first zone is $B_{2}(2 L) \backslash B_{2}(L)$ in which the function $\phi_{0}$ is roughly linearly increasing as the distance from $B_{2}(L)$ increases and satisfies

$$
\phi_{0}(x) \approx \frac{\log L}{\log N} d\left(x, B_{2}(L)\right) .
$$

The second zone is $B(N) \backslash B_{2}(2 L)$ in which $\phi_{0}$ satisfies

$$
\phi_{0}(x) \approx \frac{\log |x|}{\log N}
$$


9.4.3. Estimating $\phi_{0}$ in the case $d>2$. Because of the basic known property of $\phi_{0}$ discussed earlier, it satisfies $\phi_{0} \approx 1$ on the portion of $U_{N, L}$ which is at distance of order $N$ from $B_{2}(L)$ (the outer-part of $\left.U_{N, L}\right)$. The function $\phi_{0}$ is also bounded on $U_{N}$, uniformly in $N, L$. One key step is to find out the region in $U_{N, L}$ over which $\phi_{0}$ is bounded below by a fixed small $\epsilon$. For this purpose we use, a simple comparison with the Green's function $G(\mathbf{0}, y)=\sum_{t=0}^{\infty} K^{t}(\mathbf{0}, y)$, of the simple random walk on $\mathbb{Z}^{d}$. First, find the smallest positive $T=T(L)$ such that

$$
B_{2}(L) \subset\left\{x \in \mathbb{Z}^{d}: G(\mathbf{0}, x) \geq T\right\} .
$$

Recall that

$$
G(\mathbf{0}, x) \approx 1 /(1+|x|)^{d-2}
$$

This shows that $T \approx 1 / L^{d-2}$ (the implied constants in this estimate depend on $d$ because we are using both the Euclidean norm and the graph distance).

We are going to compare $\phi_{0}$ to a multiple of the harmonic function

$$
v(x)=1-G(\mathbf{0}, x) / T
$$

to show that $\phi \geq a v$ on $V_{N, L}$. It is clear that $v \approx 1$ when $|x|=N$ (uniformly over $N, L)$. It follows that there is a constant $a>0$, independent of $N, L$, such that $\phi_{0}-a v$ is nonnegative on the boundary of $V_{N, L}=B(N) \backslash\{z: G(\mathbf{0}, z) \geq T\}$ (the constant $a$ is chosen so that this is true on the outer-boundary whereas, on the inner-boundary, $\left.v=0, \phi_{0}>0\right)$. Since $\alpha-a v$ is superharmonic on $V_{N, L}$, it follows by the maximum principle that $\phi_{0} \geq a v$ on $V_{N, L}$. Because of the known estimate for $G$ recalled above and of the general properties of $\phi_{0}$, this shows that

$$
\phi_{0} \approx 1 \text { over } B(N) \backslash B_{2}(2 L) .
$$

All the statements and arguments given so far would work just as well if we where considering $B(N) \backslash B(L)$ instead of $B(N) \backslash B_{2}(L)$. These two cases differ only in the behavior of their respective $\phi_{0}$ near the interior boundary. For $U_{N, L}=$ $B(N) \backslash B_{2}(L)$, it is possible to show that

$$
\phi_{0}(x) \approx \frac{d\left(x, B_{2}(L)\right)}{L} .
$$

The fundamental reason for this is the (uniform) smoothness of the boundary of the Euclidean ball $B_{2}(L)$ (viewed at scale $L$ ). The result is a consequence of one of the main result in Varopoulos (2009) (see also Varopoulos, 2014, 2015).

9.5. $B(N) \backslash B(L), d=2$. Next we consider $B(N) \backslash B(L), L<N / 2$, in dimension $d=2$. We have again

$$
\beta_{0} \approx 1 /\left(N^{2} \log (N / L)\right)
$$

In the zone $B(N) \backslash B_{2}(2 L)$ (outside the yellow area in Figure 9.27), the function $\phi_{0}$ is estimated by

$$
\phi_{0}(x) \approx \frac{\log |x|}{\log N}
$$

We note here that the exact outer shape of the yellow region is unimportant (we could have drawn a diamond instead of a round ball). In order to describe the function $\phi_{0}$ is the yellow zone $\left(B_{2}(2 L) \backslash B(L)\right)$, it is convenient to split the region into eight areas, the four edges and four corners. To be more precise, see Figure 9.28, where the two red circles describes the two types of region that we will consider. 


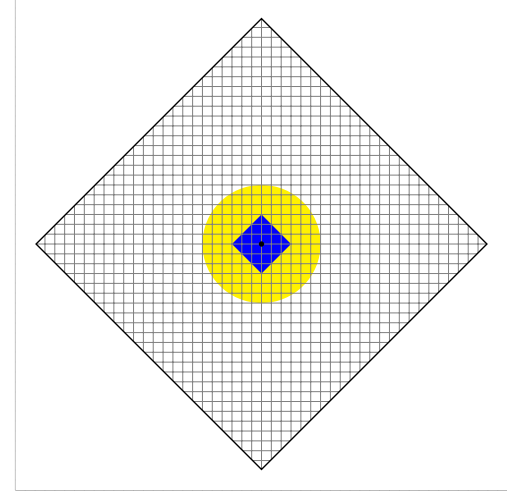

FiguRE 9.27. $B(N) \backslash B(L)$

The estimates described below are compatible when two regions intersect. In the type 1 regions, because the relevant piece of the boundary at scale $L$ is flat,

$$
\phi_{0}(x) \approx \frac{\log L}{L \log N} d(x, B(L)) .
$$

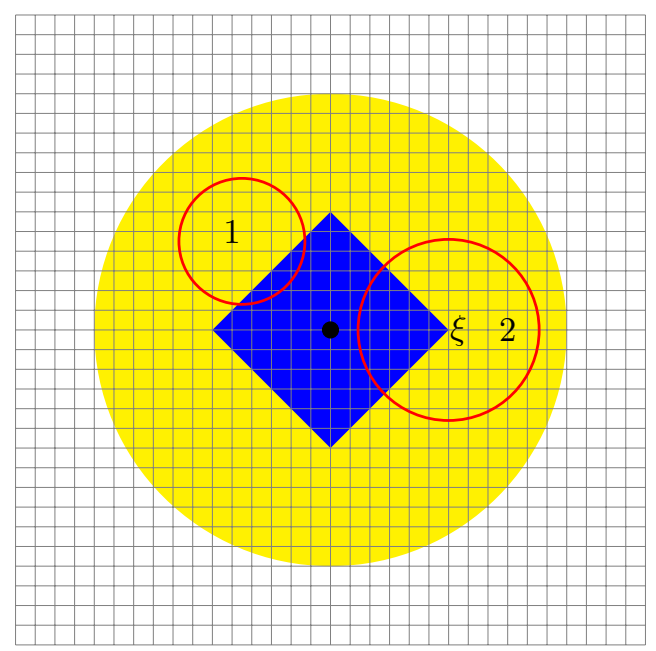

Figure 9.28. The yellow zone in $B(N) \backslash B(L)$

In the type 2 regions, centered around one of the corner of $B(L)$,

$$
\phi_{0}(x) \approx \frac{\log L}{\log N}(\rho / L)^{2 / 3} \cos (4 \theta / 3), \quad x=\left(x_{1}, x_{2}\right), x-\xi=\rho e^{i \theta}
$$

Here $\xi$ is the tip of the diamond $B(L)$ around which the region of type 2 is centered, $\theta$ is the angle in $[-\pi, \pi)$ measured from the median semi-axis through the tip. This last estimate is obtained by using the results of Varopoulos (2009) to derive the behavior of discrete harmonic function in a type 2 region from the behavior of the analogous classical harmonic function in the unbounded one-sided-cone with 
aperture $3 \pi / 2$ in $\mathbb{R}^{2}$, i.e., $\left\{(r, \theta) \in \mathbb{R}^{2}: \theta \in[0,3 \pi / 2]\right\}$ with boundary lines $\theta=0$ and $\theta=3 \pi / 2$.

\section{Summary and concluding remarks}

This article gives detailed quantitative estimates describing the behavior of Markov chains on certain finite sub-domains of a large class of underlying graphs before the chain exits the given sub-domain. There are two types of key assumptions.

The first set of assumptions concern the underlying graph (before we consider a particular sub-domain). This underlying graph belongs to a large class of graphs whose properties mimic those of the square grid $\mathbb{Z}^{m}$. This class of graphs can be defined in a variety of known equivalent different ways: it satisfies, uniformly at all scales and locations, the doubling volume condition and Poincaré inequality on balls; equivalently, the iterated kernel of simple random walk satisfies detailed two-sided "Gaussian or sub-Gaussian bounds"; or, equivalently, it satisfies a certain type of parabolic Harnack inequality for (local) positive solutions of the discrete heat equation. See the books Barlow (2017); Grigor'yan (2009) for details and pointers to the literature. It is perfectly fine for the reader to concentrate attention on the case of the square grid $\mathbb{Z}^{m}$. However, even if the reader concentrates on this special case, the techniques that are then used to study the behavior of the chain in sub-domains are the same techniques as the ones needed to understand the more general class of graphs we just alluded to.

The second set of assumptions concerns the finite sub-domains of the underlying graph that can be treated. These sub-domains are called John domains and inneruniform domains, and both are defined using metric properties. For John domains (the larger class), there is a central point $o$ and any other point of the domain can be joined to the central point $o$ by a carrot-shaped region that remains entirely contained in the domain. The inner-uniform condition (a strictly more restrictive condition) requires that any pair of point in the domain can be joined by a bananashaped region that is entirely contained in the domain. It is not easy to get a good precise understanding of the type of regions afforded by these conditions because they allow for very rugged domains (e.g., in the Euclidean plane version, the classical Koch snowflake). They do cover many interesting examples.

It is worth emphasizing here that the strength of the results obtained in this article comes from the conjunction of the two types of assumptions described above. Under these assumptions, one can describe the results of this paper by saying that any question about the behavior of the chain until it exits the given sub-domain boils down (in a technically precise and informative way) to estimating the so-called Perron-Frobenius eigenvalue and eigenfunction of the domain. Let us stress here that it is quite clear that it is necessary to understand the Perron-Frobenius pair in order to get a handle on the behavior of the chain until it exits the domain. What is remarkable is the fact that it is essentially sufficient to understand this pair in order to answer a host of seemingly more sophisticated and intricate questions. This idea is not new as it is the underlying principle of the method known as the Doob-transform technique which has been used by many authors before. Under two basic types of assumptions described above, this idea works remarkably well. In different contexts (diffusion, continuous metric measure spaces, Dirichlet forms 
and unbounded domains) this same idea is the basis for many of the developments in Pinsky (1995); Gyrya and Saloff-Coste (2011).

For inner-uniform domains, the more restrictive class of domains, the results obtained are rather detailed and complete. For John domains, the results obtained, which depend on the notion of moderate growth (see Lemma 6.2), are less detailed and leave interesting questions open.

We conclude with pointing out to further potential developments. This article focuses on the behavior before the exit time of the given finite domain. In the followup paper Diaconis et al. (2020+), we discuss, in the case of inner-uniform domains, the implications of these results on the problem of understanding the exit position. This can be framed as an extension of the classical Gambler's ruin problem. In a spirit similar to what was said above, Diaconis et al. $(2020+)$ shows how Gambler's ruin estimates on inner-uniform domains reduce to an understanding of the PerronFrobenius eigensolution on the domain. Much less is known for John domains in this direction.

Having reduced a certain number of interesting questions to the problem of estimating the Perron-Frobenius eigenfunction $\phi_{0}$ of a given finite domain, we owe the reader to observe that this task, estimating $\phi_{0}$, remains extremely difficult. There are plenty of interesting results in this direction and many more natural open problems. An illustrative example is the following: consider the cube of side length $2 N$ in $\mathbb{Z}^{3}$ with the three main coordinate axes going through the center removed; this is an inner-uniform domain and we would like to estimate the eigenfunction $\phi_{0}$. Another example, less mysterious, is to find precise estimates for $\phi_{0}$ for the graph balls in $\mathbb{Z}^{m}$ with $m \geq 3$.

For finite domains in $\mathbb{Z}^{m}$ with diameter $R$, we have proved that the key convergence parameter for the quasi-stationarity problems considered here is order $R^{2}$ for $\alpha$-inner-uniform domains and no more than $R^{2+\omega}$ for $\alpha$-John domains where $\omega \geq 0$ depends only on the dimension $m$ and John parameter $\alpha$. It is an interesting open question to decide whether or not $\omega$ can be taken to be always equal to 0 . Even if there are John domains where $\omega$ must be positive, it is clear that there is a class of John domains that is strictly larger than the class of all inner-uniform domains and for which one can take $\omega=0$. Elucidating this question is an interesting open problem in the present context and in the context of analysis in Euclidean domains.

\section{Acknowledgements}

The authors thank Tianyi Zheng for her contributions to the early stage of this work and, in particular, for pointing out that the idea of moderate growth is useful in treating John domains. We also thank the referee for their helpful remarks and encouraging us to improve the presentation of the paper. Persi Diaconis was partially supported by NSF grant DMS-1954042. Laurent Saloff-Coste was partially supported by NSF grant DMS-1707589. Kelsey Houston-Edwards was partially supported by NSF grants DMS-0739164 and DMS-1645643.

\section{Appendix A. Convex sets in $\mathbb{Z}^{d}$ with $d>2$}

To deal with higher dimensional grids $(d>2)$, let us adopt here the definition put forward by Virág (1998): a subset $U$ of the square lattice $\mathbb{Z}^{d}$ is convex if and only if there exists a convex set $C \subset \mathbb{R}^{d}$ such that $U=\left\{x \in \mathbb{Z}^{d}: d_{\infty}(x, C) \leq 1 / 2\right\}$ 
where $d_{\infty}(x, y)=\max \left\{\left|x_{i}-y_{i}\right|: 1 \leq i \leq d\right\}$. The set $C$ is called a base for $U$. We will use three distances on $\mathbb{R}^{d}$ and $\mathbb{Z}^{d}$ : the max-distance $d_{\infty}$, the Euclidean $L^{2}$ distance $d_{2}(x, y)=\sqrt{\sum_{i=1}^{d}\left|x_{i}-y_{i}\right|^{2}}$ and the $L^{1}$-distance $d_{1}(x, y)=\sum_{i=1}^{d}\left|x_{i}-y_{i}\right|$ which coincides with the graph distance on $\mathbb{Z}^{d}$.

Virág (1998) shows that, given a subset $U$ of $\mathbb{Z}^{d}$ that is convex in the sense explained above, for any two points $x, y \in U$, there is a discrete path $\gamma_{x y}=$ $\left(z_{0}, \ldots, z_{m}\right)$ in $U$ such that: (a) $z_{0}=x, z_{m}=y$; (b) $\gamma_{x y}$ is a discrete geodesic path in $\mathbb{Z}^{d}$; and (c) if $L_{x y}$ is the straight-line passing through $x$ and $y$ then each vertex $z_{i}$ on $\gamma_{x y}$ satisfies $d_{\infty}\left(z_{i}, L_{x y}\right)<1$. We will use this fact to prove the following proposition.

Proposition A.1. Let $U \subset \mathbb{Z}^{d}$ be convex in the sense explained above, with base $C$. Suppose there is a point $o$ in $U$ and positive reals $\alpha, R$ such that

$$
B_{2}(o, \alpha R) \subset C \text { and } C+B_{\infty}(0,1) \subset B_{2}(o, R),
$$

where $C+B_{\infty}(0,1)=\left\{y \in \mathbb{R}^{d}: d_{\infty}(y, C) \leq 1\right\}$. Then the set $U$ is in $J\left(o, \alpha^{\prime}, R^{\prime}\right)$ with $\alpha^{\prime}=\alpha /(6 d \sqrt{d})$ and $\alpha R \leq R^{\prime} \leq \sqrt{d} R$, where $d$ is the dimension of the underlying graph $\mathbb{Z}^{d}$.

The dimensional constants in this statement are related to the use of three metrics, namely, $d_{1}, d_{2}$ and $d_{\infty}$.

Remark A.2. In practice, this definition is more flexible than it first appears because one can choose the base $C$. Moreover, once a certain finite domain $U$ is proved to be an $\alpha_{0^{-}}$John-domain in $\mathbb{Z}^{d}$, it is easy to see that we are permitted to add and subtract in an arbitrary fashion lattice points that are at a fixed distance $r_{0}$ from the boundary $\partial U$ of $U$ in $\mathbb{Z}^{d}$, as long as we preserve connectivity. The cost is to change the John-parameter $\alpha_{0}$ to $\bar{\alpha}_{0}$ where $\bar{\alpha}_{0}$ depends only on $r_{0}$ and $\alpha_{0}$.

Proof of Proposition A.1: The convexity of $C$ (together with that of the unit cube $\left.B_{\infty}(0,1)\right)$ implies the convexity of $C^{\prime}=C+B_{\infty}(0,1)$. Thus, we know that there exist straight-line segments $l_{x}$ joining any point $x \in C^{\prime}$ to $o$, and $C^{\prime}$ is convex. By assumption (A.1) and $B_{2}(o, \alpha R) \subset C^{\prime} \subset B_{2}(o, R)$, which demonstrates that $C^{\prime} \in \mathbf{J}(\alpha R, R)$. For $x \in U$, the construction in Virág (1998) provides a discrete geodesic path $\gamma_{x}=\left(x_{0}, \ldots, x_{m_{x}}\right)$ (of length $\left.m_{x}\right)$ in $\mathbb{Z}^{d}$ joining $x$ to $o$ within the set $U$ and which stays at most $d_{\infty}$-distance 1 from $l_{x}$. As usual, we parametrize $l_{x}$ by arc-length so that $l_{x}(0)=x, l_{x}(T)=o, T=T_{x}$. For each point $x_{i} \in \gamma_{x}$, we pick a point $z_{i}$ on $l_{x}$ such that $d_{\infty}\left(x_{i}, z_{i}\right)=d_{\infty}\left(x_{i}, l_{x}\right)<1$ and define $t_{i} \in[0, T]$ by $z_{i}=l_{x}\left(t_{i}\right)$. For each $x \in U$,

$$
d_{1}(x, o) \leq \sqrt{d} d_{2}(x, o) \leq \sqrt{d} R .
$$

To obtain a lower bound on $d_{1}\left(x_{i}, \mathbb{Z}^{d} \backslash U\right)$, observe that

$$
d_{1}\left(x_{i}, \mathbb{Z}^{d} \backslash U\right) \geq d_{1}\left(x_{i}, \mathbb{R}^{d} \backslash C\right)
$$

because $C$ is contained in $U+B_{\infty}\left(0, \frac{1}{2}\right)$. By definition of $C^{\prime}, d_{1}\left(x_{i}, \mathbb{R}^{d} \backslash C\right) \geq$ $d_{1}\left(x_{i}, \mathbb{R}^{d} \backslash C^{\prime}\right)-d$. Hence, we have

$$
d_{1}\left(x_{i}, \mathbb{Z}^{d} \backslash U\right) \geq d_{2}\left(x_{i}, \mathbb{R}^{d} \backslash C^{\prime}\right)-d .
$$

Recall that $z_{i}=l_{x}\left(t_{i}\right)$ is on the line-segment from $x$ to $o$ and at $d_{\infty}$-distance less than 1 from $x_{i}$. Further, we know that $d_{2}\left(x_{i}, \mathbb{R}^{d} \backslash C^{\prime}\right) \geq \alpha t_{i}$ because $C^{\prime}$ is inner 
uniform. Also, we have

$$
t_{i}=d_{2}\left(x, z_{i}\right) \geq d_{2}\left(x, x_{i}\right)-d_{2}\left(x_{i}, z_{i}\right) \geq \frac{d_{1}\left(x, x_{i}\right)}{\sqrt{d}}-\sqrt{d}=\frac{i-d}{\sqrt{d}} .
$$

Putting these estimates together gives

$$
d_{1}\left(x_{i}, \mathbb{Z}^{d} \backslash U\right) \geq \frac{\alpha}{\sqrt{d}}\left(i-d-\frac{d \sqrt{d}}{\alpha}\right) .
$$

We claim that this implies

$$
d_{1}\left(x_{i}, \mathbb{Z}^{d} \backslash U\right) \geq \frac{\alpha}{6 d \sqrt{d}}(1+i)
$$

for all $0 \leq i \leq m_{x}$. If $i>2\left(d+\frac{d \sqrt{d}}{\alpha}\right)$ then

$$
\frac{\alpha}{\sqrt{d}}\left(i-d-\frac{d \sqrt{d}}{\alpha}\right) \geq \frac{\alpha}{\sqrt{d}}\left(\frac{i}{2}\right) \geq \frac{\alpha}{6 d \sqrt{d}}(1+i),
$$

and so the claim follows by the inequality above. Alternatively, if $i \leq 2\left(d+\frac{d \sqrt{d}}{\alpha}\right)$, then $\frac{\alpha}{6 d \sqrt{d}}(1+i) \leq 1$ and so the claim follows because $d\left(x_{i}, \mathbb{Z}^{d} \backslash U\right) \geq 1$.

\section{Appendix B. Volume doubling and moderate growth}

B.1. Doubling and moderate growth. Assume that $\mathfrak{X}$ is equipped with a positive measure $\pi$, where $\pi(A)=\sum_{x \in A} \pi(x)$ for any finite subset $A$ of $\mathfrak{X}$. (The total mass $\pi(\mathfrak{X})$ may be finite or infinite.) Denote the volume of a ball with respect to $\pi$ as

$$
V(x, r)=\pi(B(x, r)) \text {. }
$$

For any function $f$ and any ball $B$ we set

$$
f_{B}=\frac{1}{\pi(B)} \sum_{x \in B} f(x) \pi(x) .
$$

If $U$ is a finite subset of $\mathfrak{X}$, then let $\left.\pi\right|_{U}$ be the restriction of $\pi$ to $U$, i.e., $\left.\pi\right|_{U}(x)=$ $\pi(x) \mathbf{1}_{U}(x)$. We often still call this measure $\pi$. Let $\pi_{U}$ be the probability measure on $U$ that is proportional to $\left.\pi\right|_{U}$, i.e., $\pi_{U}(x)=\frac{\left.\pi\right|_{U}(x)}{Z}$ where $Z=\left.\sum_{y \in U} \pi\right|_{U}(y)$ is the normalizing constant.

Recall from Definition 3.1, that $\pi$ is doubling (with respect to $(\mathfrak{X}, \mathfrak{E})$ ) if there exists a constant $D$ (the doubling constant) such that, for all $x \in \mathfrak{X}$ and $r>0$,

$$
V(x, 2 r) \leq D V(x, r) \text {. }
$$

This property has many implications. The proofs are left to the reader.

(1) For any $x \sim y, \pi(x) \leq D \pi(y)$.

(2) For any $x \in \mathfrak{X}, \#\{y:\{x, y\} \in \mathfrak{E}\} \leq D^{2}$.

(3) For any $x \in \mathfrak{X}, r \geq s>0$ and $y \in B(x, r)$,

$$
\frac{V(x, r)}{V(y, s)} \leq D^{2}\left(\frac{\max \{1, r\}}{\max \{1, s\}}\right)^{\log _{2} D}
$$

We will need the following classic result for the case $p=2$. (For example, for the proofs of Theorems 4.6 and 4.10.) The complete proof is given here for the convenience of the reader. 
Proposition B.1. Let $(\mathfrak{X}, \mathfrak{E}, \pi)$ be doubling. For any $p \in[1, \infty)$, any real number $t \geq 1$, any finite sequence of balls $B_{i}$, and any sequence of non-negative reals $a_{i}$, we have

$$
\left\|\sum_{i} a_{i} \mathbf{1}_{t B_{i}}\right\|_{p} \leq C\left\|\sum_{i} a_{i} \mathbf{1}_{B_{i}}\right\|_{p},
$$

where $C=2\left(D^{2} p\right)^{1-1 / p} D^{1+\log _{2} t}$ and $\|f\|_{p}=\left(\sum_{\mathfrak{X}}|f|^{p} \pi\right)^{1 / p}$.

Remark B.2. For $p=1$, the result is trivial since $\pi(t B) \leq D^{\log _{2}(t)} \pi(B)$ for any ball $B$.

Proof: For any function $f$, consider the maximal function

$$
M f(x)=\sup _{B \ni x}\left\{\frac{1}{\pi(B)} \sum_{y \in B}|f(y)| \pi(y)\right\} .
$$

By Lemma B.3 below, $\|M f\|_{q} \leq C_{q}\|f\|_{q}$ for all $1<q \leq+\infty$. Also, for any ball $B$, $x \in B$ and function $h \geq 0$, we have

$$
\frac{1}{\pi(t B)} \sum_{y \in t B} h(y) \pi(y) \leq(M h)(x)
$$

and thus

$$
\frac{1}{\pi(t B)} \sum_{y \in t B} h(y) \pi(y) \leq \frac{1}{\pi(B)} \sum_{y \in B}(M h)(y) \pi(y) .
$$

Set

$$
f(y)=\sum_{i} a_{i} \mathbf{1}_{t B_{i}}(y) \text { and } g(y)=\sum_{i} a_{i} \mathbf{1}_{B_{i}}(y) .
$$

It suffices to prove that, for all functions $h \geq 0,\left|\sum f h \pi\right| \leq C\|g\|_{p}\|h\|_{q}$, where $1 / p+1 / q=1$. Note that

$$
\begin{aligned}
\sum_{y \in \mathfrak{X}} f(y) h(y) \pi(y) & =\sum_{i} a_{i} \sum_{y \in t B_{i}} h(y) \pi(y) \\
& \leq \sum_{i} a_{i} \frac{\pi\left(t B_{i}\right)}{\pi\left(B_{i}\right)} \sum_{y \in B_{i}}(M h)(y) \pi(y) \\
& \leq D^{1+\log _{2} t} \sum_{i} a_{i} \sum_{y \in B_{i}}(M h)(y) \pi(y) \\
& =D^{1+\log _{2} t} \sum_{y \in \mathfrak{X}} \sum_{i} a_{i} \mathbf{1}_{B_{i}}(M h)(y) \pi(y) \\
& \leq D^{1+\log _{2} t}\|g\|_{p}\|M h\|_{q} \\
& \leq C_{q} D^{1+\log _{2}(t)}\|g\|_{p}\|h\|_{q} .
\end{aligned}
$$

Applying this fact with $h=f^{p / q}$ proves the desired result.

Lemma B.3. For any $q \in(1,+\infty]$ and any $f$, the maximal function $M$ satisfies $\|M f\|_{q} \leq C_{q}\|f\|_{q}$ with $C_{q}=2\left(D^{2} p\right)^{1-1 / p}$ where $1 / p+1 / q=1$.

Proof: Consider the set $V_{\lambda}^{f}=\{x: M f(x)>\lambda\}$. By definition, for each $x \in V_{\lambda}^{f}$ there is a ball $B_{x}$ such that $\frac{1}{\pi\left(B_{x}\right)} \sum_{y \in B_{x}}|f(y)| \pi(y)>\lambda$. Form

$$
\mathcal{B}=\left\{B_{x}: x \in V_{\lambda}^{f}\right\}
$$


and extract from it a set of disjoint balls $B_{1}, \ldots, B_{q}$ so that $B_{1}$ has the largest possible radius among all balls in $\mathcal{B}, B_{2}$ has the largest possible radius among all balls in $\mathcal{B}$ which are disjoint from $B_{1}$. At stage $i$, the ball $B_{i}$ is chosen to have the largest possible radius among the balls $B_{x}$ which are disjoint from $B_{1}, \ldots, B_{i-1}$. We stop when no such balls exist.

We claim that the balls $3 B_{i}$ cover $V_{\lambda}^{f}$, where $1 \leq i \leq q$ and $q$ is the size of $\mathcal{B}$. For any $x \in V_{\lambda}^{f}$, we have $B_{x}=B(z, r)$, for some $z$ and $r$, and $B(z, r) \cap\left(\cup_{1}^{q} B_{i}\right) \neq \emptyset$. By construction if $j$ is the first subscript such that there exists $y \in B(z, r) \cap B_{j}, r$ must be no larger than the radius of $B_{j}$. This implies $z \in 2 B_{j}$ and $x \in 3 B_{j}$.

It follows from the fact that $3 B_{i}$ cover $V_{\lambda}^{f}$ that

$$
\pi\left(V_{\lambda}^{f}\right) \leq D^{2} \sum_{i=1}^{q} \pi\left(B_{i}\right) \leq D^{2} \lambda^{-1} \sum_{i=1}^{q} \sum_{x \in B_{i}}|f(x)| \pi(x) \leq D^{2} \lambda^{-1} \sum_{x \in \mathfrak{X}}|f(x)| \pi(x) .
$$

Next observe that $M f \leq M\left(f \mathbf{1}_{\{|f|>\lambda / 2\}}\right)+\lambda / 2$ and thus

$$
\{x: M f(x)>\lambda\} \subset\left\{x: M\left(f \mathbf{1}_{\{|f|>\lambda / 2\}}\right)(x)>\lambda / 2\right\} .
$$

Therefore $\pi(M f>\lambda) \leq 2 D^{2} \lambda^{-1} \sum_{\{x:|f(x)|>\lambda / 2\}}|f(x)| \pi(x)$. Finally, recall that

$$
\|h\|_{q}^{q}=q \int_{0}^{\infty} \pi(h>\lambda) \lambda^{q-1} d \lambda
$$

for $h \geq 0$. This gives

$$
\|M f\|_{q}^{q} \leq 2 q D^{2} \sum_{x \in \mathfrak{X}} \int_{0}^{2|f(x)|} \lambda^{q-2} d \lambda|f(x)| \pi(x)=\frac{q D^{2} 2^{q}}{q-1} \sum_{x \in \mathfrak{X}}|f(x)|^{q} \pi(x) .
$$

This gives $C_{q}=2 D^{2 / q}\left(\frac{1}{1-1 / q}\right)^{1 / q}$. If $1 / p+1 / q=1$ then $C_{q}=2\left(D^{2} p\right)^{1-1 / p}$.

The following notion of moderate growth is key to our approach. It was introduced in Diaconis and Saloff-Coste (1994) for groups and in Diaconis and SaloffCoste (1996) for more general finite Markov chains. The reader will find many examples there. It is used below repeatedly, in particular, in Lemma 6.2 and Theorems 6.4-6.6-6.7, and in Theorems 7.14-7.17-7.23.

Definition B.4. Assume that $\mathfrak{X}$ is finite. We say that $(\mathfrak{X}, \mathfrak{E}, \pi)$ has $(a, \nu)$-moderate volume growth if the volume of balls satisfies

$$
\forall r \in(0, \operatorname{diam}], \quad \frac{V(x, r)}{\pi(\mathfrak{X})} \geq a\left(\frac{1+r}{\operatorname{diam}}\right)^{\nu},
$$

where diam $=\sup \left\{\left|\gamma_{x y}\right|: x, y \in \mathfrak{X}\right\}$ is the maximum of path lengths $\left|\gamma_{x y}\right|$ with $\gamma_{x y}$ the shortest path between $x, y \in \mathfrak{X}$.

Remark B.5. When $\mathfrak{X}$ is finite and $\pi$ is $D$-doubling then $(\mathfrak{X}, \mathfrak{E}, \pi)$ has $\left((D)^{-2}, \log _{2} D\right)$-moderate growth because

$$
\frac{V(x, s)}{\pi(\mathfrak{X})}=\frac{V(x, s)}{V(x, \operatorname{diam})} \geq D^{-1}\left(\frac{\max \{1, s\}}{\operatorname{diam}}\right)^{\log _{2} D} \geq D^{-2}\left(\frac{1+s}{\operatorname{diam}}\right)^{\log _{2} D} .
$$

Because of this remark, moderate growth can be seen as a generalization of the doubling condition. It implies that the size of $\mathfrak{X}$ (as measured by $\pi(\mathfrak{X})$ ) is bounded by a power of the diameter (this can be viewed as a "finite dimension" condition and a rough upper bound on volume growth). It also implies that the measure of

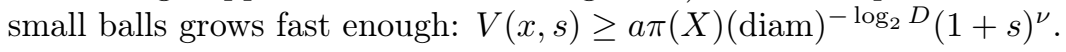




\section{References}

Aikawa, H. Boundary Harnack principle and Martin boundary for a uniform domain. J. Math. Soc. Japan, 53 (1), 119-145 (2001). MR1800526.

Aikawa, H. Martin boundary and boundary Harnack principle for non-smooth domains [MR1962228]. In Selected papers on differential equations and analysis, volume 215 of Amer. Math. Soc. Transl. Ser. 2, pp. 33-55. Amer. Math. Soc., Providence, RI (2005). MR2186550.

Aikawa, H. Equivalence between the boundary Harnack principle and the Carleson estimate. Math. Scand., 103 (1), 61-76 (2008). MR2464701.

Aikawa, H. Intrinsic ultracontractivity via capacitary width. Rev. Mat. Iberoam., 31 (3), 1041-1106 (2015). MR3420485.

Ancona, A. Principe de Harnack à la frontière et théorème de Fatou pour un opérateur elliptique dans un domaine lipschitzien. Ann. Inst. Fourier (Grenoble), 28 (4), 169-213, x (1978). MR513885.

Bañuelos, R., Bass, R. F., and Burdzy, K. Hölder domains and the boundary Harnack principle. Duke Math. J., 64 (1), 195-200 (1991). MR1131398.

Barlow, M. T. Random walks and heat kernels on graphs, volume 438 of London Mathematical Society Lecture Note Series. Cambridge University Press, Cambridge (2017). ISBN 978-1-107-67442-4. MR3616731.

Barlow, M. T. and Bass, R. F. Stability of parabolic Harnack inequalities. Trans. Amer. Math. Soc., 356 (4), 1501-1533 (2004). MR2034316.

Barlow, M. T., Coulhon, T., and Kumagai, T. Characterization of sub-Gaussian heat kernel estimates on strongly recurrent graphs. Comm. Pure Appl. Math., 58 (12), 1642-1677 (2005). MR2177164.

Berkolaiko, G. and Kuchment, P. Introduction to quantum graphs, volume 186 of Mathematical Surveys and Monographs. American Mathematical Society, Providence, RI (2013). ISBN 978-0-8218-9211-4. MR3013208.

Billera, L. J. and Diaconis, P. A geometric interpretation of the Metropolis-Hastings algorithm. Statist. Sci., 16 (4), 335-339 (2001). MR1888448.

Burkholder, D. L. Exit times of Brownian motion, harmonic majorization, and Hardy spaces. Advances in Math., 26 (2), 182-205 (1977). MR474525.

Carleson, L. On the existence of boundary values for harmonic functions in several variables. Ark. Mat., 4, 393-399 (1962) (1962). MR159013.

Cattaneo, C. The spectrum of the continuous Laplacian on a graph. Monatsh. Math., 124 (3), 215-235 (1997). MR1476363.

Cipriani, F. Intrinsic ultracontractivity of Dirichlet Laplacians in nonsmooth domains. Potential Anal., 3 (2), 203-218 (1994). MR1269281.

Collet, P., Martínez, S., and San Martín, J. Quasi-stationary distributions. Markov chains, diffusions and dynamical systems. Springer, Heidelberg (2013). ISBN 978-3-642-33130-5; 978-3-642-33131-2. MR2986807.

Coulhon, T. Random walks and geometry on infinite graphs. In Lecture notes on analysis in metric spaces (Trento, 1999), Appunti Corsi Tenuti Docenti Sc., pp. 5-36. Scuola Norm. Sup., Pisa (2000). MR2023121.

Dahlberg, B. E. J. Estimates of harmonic measure. Arch. Rational Mech. Anal., 65 (3), 275-288 (1977). MR466593.

Davies, E. B. Criteria for ultracontractivity. Ann. Inst. H. Poincaré Phys. Théor., 43 (2), 181-194 (1985). MR817534. 
Davies, E. B. and Simon, B. Ultracontractivity and the heat kernel for Schrödinger operators and Dirichlet Laplacians. J. Funct. Anal., 59 (2), 335-395 (1984). MR766493.

Davis, B. Intrinsic ultracontractivity and the Dirichlet Laplacian. J. Funct. Anal., 100 (1), 162-180 (1991). MR1124297.

DeBlassie, R. D. Exit times from cones in $\mathbf{R}^{n}$ of Brownian motion. Probab. Theory Related Fields, 74 (1), 1-29 (1987). MR863716.

Delmotte, T. Inégalité de Harnack elliptique sur les graphes. Colloq. Math., 72 (1), 19-37 (1997). MR1425544.

Delmotte, T. Parabolic Harnack inequality and estimates of Markov chains on graphs. Rev. Mat. Iberoamericana, 15 (1), 181-232 (1999). MR1681641.

Denisov, D. and Wachtel, V. Random walks in cones. Ann. Probab., 43 (3), 9921044 (2015). MR3342657.

Diaconis, P., Houston-Edwards, K., and Saloff-Coste, L. Gambler's ruin estimates on finite inner uniform domains $(2020+)$. To appear in Ann. Appl. Probab.

Diaconis, P. and Miclo, L. On quantitative convergence to quasi-stationarity. Ann. Fac. Sci. Toulouse Math. (6), 24 (4), 973-1016 (2015). MR3434264.

Diaconis, P. and Saloff-Coste, L. Comparison techniques for random walk on finite groups. Ann. Probab., 21 (4), 2131-2156 (1993a). MR1245303.

Diaconis, P. and Saloff-Coste, L. Comparison theorems for reversible Markov chains. Ann. Appl. Probab., 3 (3), 696-730 (1993b). MR1233621.

Diaconis, P. and Saloff-Coste, L. Moderate growth and random walk on finite groups. Geom. Funct. Anal., 4 (1), 1-36 (1994). MR1254308.

Diaconis, P. and Saloff-Coste, L. Nash inequalities for finite Markov chains. $J$. Theoret. Probab., 9 (2), 459-510 (1996). MR1385408.

Diaconis, P. and Saloff-Coste, L. What do we know about the Metropolis algorithm? J. Comput. System Sci., 57 (1), 20-36 (1998). MR1649805.

Folz, M. Volume growth and stochastic completeness of graphs. Trans. Amer. Math. Soc., 366 (4), 2089-2119 (2014). MR3152724.

Fukushima, M., Oshima, Y., and Takeda, M. Dirichlet forms and symmetric Markov processes, volume 19 of De Gruyter Studies in Mathematics. Walter de Gruyter \& Co., Berlin, extended edition (2011). ISBN 978-3-11-021808-4. MR2778606.

Grigor'yan, A. Heat kernel and analysis on manifolds, volume 47 of AMS/IP Studies in Advanced Mathematics. American Mathematical Society, Providence, RI; International Press, Boston, MA (2009). ISBN 978-0-8218-4935-4. MR2569498.

Grigor'yan, A. Introduction to analysis on graphs, volume 71 of University Lecture Series. American Mathematical Society, Providence, RI (2018). ISBN 978-14704-4397-9. MR3822363.

Gyrya, P. and Saloff-Coste, L. Neumann and Dirichlet heat kernels in inner uniform domains. Astérisque, (336) (2011). MR2807275.

Houston-Edwards, K. Discrete Heat Kernel Esimates on Inner Uniform Domains. Ph.D. thesis, Cornell University (2018).

Jerison, D. The Poincaré inequality for vector fields satisfying Hörmander's condition. Duke Math. J., 53 (2), 503-523 (1986). MR850547.

Kemeny, J. G., Snell, J. L., and Knapp, A. W. Denumerable Markov chains. With a chapter on Markov random fields, by David Griffeath. Springer-Verlag, New York-Heidelberg-Berlin, Graduate Texts in Mathematics, No. 40, second edition 
(1976). MR0407981.

Kemper, J. T. A boundary Harnack principle for Lipschitz domains and the principle of positive singularities. Comm. Pure Appl. Math., 25, 247-255 (1972). MR293114.

Levin, D. A. and Peres, Y. Markov chains and mixing times. American Mathematical Society, Providence, RI (2017). ISBN 978-1-4704-2962-1. MR3726904.

Lierl, J. Scale-invariant boundary Harnack principle on inner uniform domains in fractal-type spaces. Potential Anal., 43 (4), 717-747 (2015). MR3432457.

Lierl, J. Parabolic Harnack inequality on fractal-type metric measure Dirichlet spaces. Rev. Mat. Iberoam., 34 (2), 687-738 (2018). MR3809455.

Lierl, J. and Saloff-Coste, L. The Dirichlet heat kernel in inner uniform domains: local results, compact domains and non-symmetric forms. J. Funct. Anal., 266 (7), 4189-4235 (2014a). MR3170207.

Lierl, J. and Saloff-Coste, L. Scale-invariant boundary Harnack principle in inner uniform domains. Osaka J. Math., 51 (3), 619-656 (2014b). MR3272609.

Liu, J. S. Monte Carlo strategies in Scientific Computing. Springer Series in Statistics. Springer, New York (2008). ISBN 978-0-387-76369-9; 0-387-95230-6. MR2401592.

Maheux, P. and Saloff-Coste, L. Analyse sur les boules d'un opérateur souselliptique. Math. Ann., 303 (4), 713-740 (1995). MR1359957.

Martio, O. and Sarvas, J. Injectivity theorems in plane and space. Ann. Acad. Sci. Fenn. Ser. A I Math., 4 (2), 383-401 (1979). MR565886.

Norris, J. R. Markov chains, volume 2 of Cambridge Series in Statistical and Probabilistic Mathematics. Cambridge University Press, Cambridge (1998). ISBN 0-521-48181-3. MR1600720.

Pang, C. Y. A. Lumpings of algebraic Markov chains arise from subquotients. $J$. Theoret. Probab., 32 (4), 1804-1844 (2019). MR4020688.

Pinsky, R. G. Positive harmonic functions and diffusion, volume 45 of Cambridge Studies in Advanced Mathematics. Cambridge University Press, Cambridge (1995). ISBN 0-521-47014-5. MR1326606.

Revuz, D. and Yor, M. Continuous martingales and Brownian motion, volume 293 of Grundlehren der Mathematischen Wissenschaften [Fundamental Principles of Mathematical Sciences]. Springer-Verlag, Berlin, third edition (1999). ISBN 3540-64325-7. MR1725357.

Saloff-Coste, L. Lectures on finite Markov chains. In Lectures on probability theory and statistics (Saint-Flour, 1996), volume 1665 of Lecture Notes in Math., pp. 301-413. Springer, Berlin (1997). MR1490046.

Saloff-Coste, L. Simple examples of the use of Nash inequalities for finite Markov chains. In Stochastic geometry (Toulouse, 1996), volume 80 of Monogr. Statist. Appl. Probab., pp. 365-400. Chapman \& Hall/CRC, Boca Raton, FL (1999). MR1673142.

Saloff-Coste, L. Aspects of Sobolev-type inequalities, volume 289 of London Mathematical Society Lecture Note Series. Cambridge University Press, Cambridge (2002). ISBN 0-521-00607-4. MR1872526.

Spitzer, F. Principles of random walk. Springer-Verlag, New York-Heidelberg, second edition (1976). MR0388547.

Varopoulos, N. T. The discrete and classical Dirichlet problem. Milan J. Math., 77, 397-436 (2009). MR2578884. 
Varopoulos, N. T. The central limit theorem in Lipschitz domains. Boll. Unione Mat. Ital., 7 (2), 103-156 (2014). MR3238537.

Varopoulos, N. T. The discrete and classical Dirichlet problem: Part II. Milan J. Math., 83 (1), 1-20 (2015). MR3347389.

Virág, B. Random walks on finite convex sets of lattice points. J. Theoret. Probab., 11 (4), 935-951 (1998). MR1660887.

Wu, J. M. G. Comparisons of kernel functions, boundary Harnack principle and relative Fatou theorem on Lipschitz domains. Ann. Inst. Fourier (Grenoble), 28 (4), 147-167, vi (1978). MR513884. 